UNDERGRADUATE RESEARCH IN NATURAL AND CLINICAL SCIENCE AND TECHNOLOGY (URNCST) JOURNAL

Read more URNCST Journal articles and submit your own today at: https://www.urncst.com

CONFERENCE ABSTRACT BOOK

OPEN ACCESS

\title{
Conference Abstract Booklet: 3rd Student Medical Summit 2020
}

Liam Shaw [1]*, Xiaoshan Pan [1], Ned Quirke [1]

[1] UCD School of Medicine and Medical Sciences, University College Dublin, Belfield, Dublin 4, Ireland

*Corresponding Author: liam.shaw@ucdconnect.ie

\begin{abstract}
The following abstracts are those that we presented at the research competition of the Student Medical Summit held in the Talbot Hotel Stillorgan on the 15th February 2020. For more information about the Student Medical Summit visit www.studentmedicalsummit.org or contactinfo@studentmedicalsummit.org
\end{abstract}

Keywords: biomedical research; medical research; poster competition; SMS; undergraduate research conference

Table of Contents

Abstracts of Poster Presentations

pg. A01-A45

\section{Conference Abstracts}

Note: These abstracts have been reproduced directly from the material supplied by the authors, without editorial alteration by the staff of the URNCST Journal. Insufficiencies of preparation, grammar, spelling, style, syntax, and usage are the authors.

\section{$\underline{\text { Poster Abstracts }}$}

1. Disruption in expression of circadian rhythm genes following mild traumatic brain injury in children Catherine V Stacey [1], Emer Ryan [1-3], Lynne A Kelly [1, 2], Eimear Duff [1], Eleanor J Molloy [1-5]

[1] Discipline of Paediatrics, Trinity College, the University of Dublin, Ireland

[2] Trinity Translational Medicine Institute, St James Hospital, Dublin 8, Dublin, Ireland

[3] Children's Hospital Ireland (CHI) at Tallaght, Cooley Rd, Crumlin, Dublin, D12 N512, Ireland

[4] Neonatology, CHI at Crumlin, Dublin, Ireland

[5] Paediatrics, Coombe Women's and Infant's University Hospital, Dublin, Ireland

Introduction: Traumatic brain injury (TBI) is a common occurrence in children. Patients recovering from TBI are prone to sleep-wake cycle disturbances [1], which indicates disruption of the circadian rhythm mechanisms that regulates sleep [2]. In this study, we evaluated the expression of circadian gene transcription following mild Traumatic Brain Injury (mTBI) to understand the processes involved in sleep disturbance following mTBI.

Methods: Whole blood was analysed from children with mild TBI within 24 hours of injury (n=13) \& at two weeks post injury $(n=7)$ compared to healthy paediatric controls at baseline $(n=17)$. Whole blood RNA was isolated, cDNA synthesized \& analysed by quantitative PCR for the expression of CLOCK, CRY, BMAL \& REVERB $\alpha$.

Results: The mean (SD) age of Controls and mTBI cohorts was $7.9(+/-4.6)$, and $11.3(+/-4.5)$ years, respectively. CLOCK expression increased between controls and mTBI, both at baseline $\&$ at 2 weeks post injury $(p=0.00002, p=0.006)$. CRY 1 expression increased at 2 weeks $(\mathrm{p}=0.001)$. REVERB-alpha expression decreased at baseline compared to controls $(\mathrm{p}=0.002)$. BMAL expression increased at presentation compared to controls $(\mathrm{p}=0.02)$.

Conclusion: We have shown significant disruption in circadian rhythm gene expression following mTBI. Injury-induced changes in the expression of CLOCK, CRY, BMAL \& REVERB $\alpha$ imply that TBI disrupts the timing of the central master clock in the anterior hippocampus controlling circadian rhythm. Future study is warranted to evaluate the impact of a chronological regulator such as Melatonin on this patient cohort and may elucidate future treatment potentials. 
UNDERGRADUATE RESEARCH IN NATURAL AND CLINICAL SCIENCE AND TECHNOLOGY (URNCST) JOURNAL

Read more URNCST Journal articles and submit your own today at: https://www.urncst.com

\section{References}

[1] Ouellet MC, Beaulieu-Bonneau S, Morin CM. Sleep-wake disturbances after traumatic brain injury. Lancet Neurol. 2015 Jul; 14(7): 746-57. https://doi.org/10.1016/S1474-4422(15)00068-X

[2] Gagner C, Landry-Roy C, Lainé F, Beauchamp MH. Sleep-Wake Disturbances and Fatigue after Pediatric Traumatic Brain Injury: A Systematic Review of the Literature. $J$ Neurotrauma. 2015 Oct 15; 32(20): 1539-52. https://doi.org/10.1089/neu.2014.3753

2. Retinal degeneration is attenuated by SARM in the novel double knockout mouse model $\mathbf{r h o}^{-/-} \mathbf{s a r m}^{-/-}$, Shane Mulvihill, BSc Student [1], Ema Ozaki, PhD [2], Sarah L. Doyle, PhD [3]

[1] School of Medicine, University of Dublin Trinity College, Dublin, Ireland

[2] Ocular Genetics Unit, Smurfit Institute of Genetics, Dublin 2, Ireland

[3] National Children's Research Centre, Our Lady's Children's Hospital, Dublin 12, Ireland

Introduction: SARM (sterile alpha and armadillo motif-containing protein) is a multifunctional cytosolic protein. Recent studies have implicated SARM's essential effector role in Wallerian degeneration, mediating subcellular axon death during injury and disease. Here, SARM cleaves and depletes local NAD ${ }^{+}$, initiating pro-apoptotic features under certain conditions, eventually inducing whole neuron "Sarmoptosis" [1]. SARM and NAD" interaction unifies this axon damage model, involved in neuropathies and neurodegenerative disease, yielding new insight for future therapeutic interventions. Novel research has also established the depletion of $\mathrm{NAD}^{+}$as a common feature among several mouse models of degenerative retinopathy. Additionally, retinal SARM RNA is expressed in rhodopsin knockout $\left(r h o^{-/}\right)$mice, a model characterised by rapid degeneration of rod photoreceptors within 12 weeks [2].

Methods: Given these observations, $\mathrm{rho}^{-/} \mathrm{sarm}^{-/}$double knockout mice were generated, to investigate the role of SARM in a model of severe degenerative retinopathy. Additionally, $661 \mathrm{~W}$ photoreceptor cells were treated with $\mathrm{NAD}^{+}$and sodium iodate to investigate oxidative stress-induced cytotoxicity.

Results: Histological examination suggested $\mathrm{rho}^{-/} \mathrm{sarm}^{-/}$mice retain higher quantities of rod photoreceptors by 12 weeks, supported by invivo OCT retinal scans demonstrating thicker outer nuclear layers in comparison to rho ${ }^{-/-}$mice. Each model exhibited secondary degeneration of cone photoreceptors, while differential localization of microglia was observed in the $\mathrm{rho}^{-/} \mathrm{sarm}^{-/-}$retina after 12 weeks. Finally, oxidative stress-induced cytotoxicity by sodium iodate was shown to be rescued by $\mathrm{NAD}^{+}$treatment in $661 \mathrm{~W}$ photoreceptor cells.

Conclusion: The absence of SARM may confer slower degeneration in this model of severe retinopathy, and further investigation is imperative.

\section{References}

[1] Lin JB, Kubota S, Ban N, Yoshida M, Santeford A, Sene A, Nakamura R, Zapata N, Kubota M, Tsubota K, Yoshino J, Imai SI, Apte RS. NAMPT-Mediated NAD (+) Biosynthesis Is Essential for Vision In Mice. Cell Rep. 2016 Sep 27; 17(1): 69-85. https://doi.org/10.1016/j.celrep.2016.08.073

[2] Humphries MM, Rancourt D, Farrar GJ, Kenna P, Hazel M, Bush RA, Sieving PA, Sheils DM, McNally N, Creighton P, Erven A, Boros A, Gulya K, Capecchi MR, Humphries P. Retinopathy induced in mice by targeted disruption of the rhodopsin gene. Nat Genet. 1997 Feb; 15(2): 216-9. https://doi.org/10.1038/ng0297-216

\section{Community first responders: Motivations of student volunteers Andrew M Namespetra, MB, BCh, BAO Student [1] \\ [1] School of Medicine, University College Cork, Cork, Co. Cork, Ireland}

Introduction: Community First Responder (CFR) organizations are composed of lay volunteers that provide basic pre-hospital life support to victims of medical emergencies [1]. University College Cork (UCC) CFR is the first student-led CFR organization Ireland, established in 2018 [2]. This study aims to explore the motives and experiences of UCC students to volunteer in this CFR organization. The specific research questions include: How does academic background influence the motivation of students participating in a CFR group? Does their self-confidence in responding to emergencies change with experience and training?

Methods: This is a mixed methods study and participants are UCC students volunteering in the CFR group. Quantitative data is collected by anonymous paper questionnaires administered before and after CFR certification and training. Qualitative data will be collected by focus group sessions or interviews, during which discussion surrounding volunteer experience is facilitated by a question framework. The narratives will be digital audio-recorded and subsequently transcribed verbatim. Thematic analysis of the transcripts will be conducted to elucidate themes.

Shaw et al. | URNCST Journal (2020): Volume 4, Issue 3

DOI Link: https://doi.org/10.26685/urnest.182 
UNDERGRADUATE RESEARCH IN NATURAL AND CLINICAL SCIENCE AND TECHNOLOGY (URNCST) JOURNAL Read more URNCST Journal articles and submit your own today at: https://www.urncst.com

Results: Based on the initial questionnaires, the strongest reasons for joining UCC CFR are: improving CPR skills, helping people, and community service. However, medical students and non-medical differ in their level of confidence at the onset of volunteering. Interviews with responders have not yet commenced, but the results will be reported following thematic analysis. Conclusion: Both medical and non-medical students have common motives for volunteering in a student-led CFR group. The interviews will gain further insight into the individual experiences of the student volunteers.

\section{References}

[1] Barry T, Doheny MC, Masterson S, Conroy N, Klimas J, Segurado R, et al. Community first responders for out-ofhospital cardiac arrest in adults and children. Cochrane Database of Systematic Reviews 2019, Issue 7. Art. No.: CD012764. https://doi.org/10.1002/14651858.CD012764.pub2

[2] Mathews D, Lesko K, O’Callaghan C, Hayes A, Deasy, C. O’Donnell C. Medical students as Community First Responders The UCC CFR Project. Poster presented at IAEM-Irish Association for Emergency Medicine; 7-8 ${ }^{\text {th }}$ November 2019; Sligo, Ireland.

4. General practitioners' views of advance care planning: A questionnaire-based study

Karendeep K. Somal, Graduate Entry Medicine Student [1], Tony Foley, General Practitioner [2]

[1] Department of Medicine, University College Cork, Cork, Ireland, T12 AK54

[2] Department of General Practice, University College Cork, Cork, Ireland T12 XF62

Introduction: Technological and medical advancements have improved the average life expectancy, which has augmented the need to understand end of life (EOL) care. Advance care plans (ACPs) allow individuals to make plans for future healthcare practices for a time when they no longer have the capacity to make such decisions. It has been suggested that general practitioners (GPs) have the ideal relationship with their patients to facilitate this process. However, ACP uptake still remains low, prompting the need to understand the views of GPs in implementing ACPs. The aim of this study was to assess the knowledge and attitudes of GPs regarding ACPs, and to identify factors hindering their implementation effectively into practice. Methods: A cross-sectional study was conducted with GPs in county Cork and Kerry. A paper-based questionnaire was adapted from two previously published studies, piloted and posted to 237 GPs.

Results: A total of 75 questionnaires were completed, resulting in a response rate of $31.7 \%$. Findings revealed that there is currently an insufficient understanding of ACPs. The principal barriers were categorized into 3 broad categories: physicianassociated, patient-associated and healthcare-system associated.

Conclusion: The findings indicate that GPs in county Cork and Kerry had a positive attitude toward ACPs. In order to improve their employment workshops and online courses should be developed to increase GPs' knowledge and confidence. Additionally, the healthcare system should be adapted to facilitate these EOL care processes to take place, such as raising awareness of ACPs, facilitating information transfer between healthcare facilities, and providing adequate resources.

\section{Paediatric major trauma; demographics, management and outcomes at Cork Liadan O'Sullivan [1], Conor Deasy [2] \\ [1] Final year Medical student, UCC Floor 2, Paediatric Unit, Cork University Hospital, Wilton, Cork, Ireland \\ [2] Project supervisor and Emergency Medicine Consultant, CUH Wilton, Cork, Ireland}

\section{Introduction:}

Objectives - To establish the demographics, injury patterns, management and outcomes of paediatric major trauma (PT) patients at Cork University Hospital (CUH).

Methods: This was a retrospective descriptive analysis of patients under the age of sixteen, who fulfilled NOCA's Major Trauma Audit inclusion criteria. Data from all CUH PT cases that were recorded in the Trauma Audit and Research Network (TARN) database from January 2014 to July 2018 were examined. Demographic profiles, types of injuries, brief management details, length of stay (LOS) and outcomes of these patients were analysed.

Results: A total of 163 patients were included who sustained life threatening or life changing trauma; mean age of 9 years (SD: 4.8 years) and the majority $(62 \%)$ were male. The most common mechanism of injury was falls $<2 \mathrm{~m}(35 \%)$. Limb injuries occurred in 74 patients (45\%). Sixty-two percent of head injuries $(n=47)$ were isolated. The mean injury severity score (ISS) was 12 (SD: 7). The median LOS was five days (IQR: 3-8 days). Four patients died (mortality rate 2\%), all male, two due to head injury, two due to asphyxia. 
UNDERGRADUATE RESEARCH IN NATURAL AND CLINICAL SCIENCE AND TECHNOLOGY (URNCST) JOURNAL Read more URNCST Journal articles and submit your own today at: https://www.urncst.com

Conclusion: The annual number of PT presentations to CUH, including road trauma cases, remains roughly constant. Limbs, followed by head, were the most common body regions to sustain injuries. A wider study including all 28 receiving hospitals that contribute data to Major Trauma Audit may reveal urban rural differences as well as paediatric hospitals versus mixed hospitals differences in performance and outcomes.

6. The effect of medicinal cannabis on quality of life in the management of non-cancer chronic pain: A systematic review

Bernard Chew, MB, BCh, BAO Student [1], Laoise Geoghegan, MB, BCh, BAO Student [1], Seongseon Kim, MB, BCh, BAO Student [1], Sorcha Murray, MB, BCh, BAO Student [1], K. Ewomazino Oderoha, MB, BCh, BAO Student [1], Molly O'Shea, MB, BCh, BAO Student [1], Cormac Kennedy, MB, BAO, BCh, BSc (Pharm), HDip (BS), MPSI, MRCPI, PhD [2]

[1] School of Medicine, Trinity College Dublin, Dublin, Ireland

[2] Department of Pharmacology and Therapeutics, St James's Hospital, Dublin, Ireland

Introduction: Medicinal cannabis (MC) is an emerging therapeutic option for non-cancer chronic pain. However, its effects on a patient's quality of life are still widely unknown, and its use still illegal in many countries. The aim of this systematic review was to examine the results of randomised controlled trials (RCTs) that study the effect of medicinal cannabis on quality of life in the treatment of non-cancer chronic pain.

Methods: This study was performed in accordance with the Preferred Reporting Items for Systematic Reviews and Meta Analyses (PRISMA) protocol and was registered with PROSPERO. A search of Cochrane Library, MEDLINE, Embase and Web of Science was undertaken for literature published on the use of medicinal cannabis for the treatment of non-malignant chronic pain, and its effect on quality of life. Relevant data was extracted from studies and methodological quality assessed using the Cochrane Risk of Bias tool.

Results: The search identified 659 research articles. Six studies were selected following application of the inclusion and exclusion criteria. The qualitative review of these studies showed methodological quality was relatively high. The included studies show an overall improvement in quality of life with the use of medicinal cannabis, this reached statistical significance in four studies. Five of the studies favoured medicinal cannabis over comparators, while one RCT favoured the use of dihydrocodeine.

Conclusion: The findings of this review suggest that while medicinal cannabis has shown to improve quality of life, the topic is not fully understood. Further RCTs are essential for future developments.

7. Suffering in Samos: A survey of the medical challenges faced by refugees stranded on Samos, Greece Conor T. O'Brien, Medical Student [1], Anna CT. Gordon, MSc, Medical Student [2], Albane Girma Medical Student [3]

[1] School of Medicine, University College Dublin, Dublin 4, Ireland

[2] School of Medicine, University of Sheffield, Western Bank, Sheffield S10 2TN, United Kingdom

[3] Faculty of Medicine, University of Angers, France

Introduction: Thousands of refugees from Africa and the Middle East are held in camps along the European border while their asylum applications are processed. This study aimed to develop a profile of the refugee population on the Greek Island of Samos where the camp is at almost 7 times its intended capacity [1]. In particular the study aimed to determine the health status of refugees to better inform government and NGO policy.

Methods: The anonymous 34-question survey was drafted in June 2019 and translated into French, Arabic and Farsi. In July 2019 it was distributed at the clinic run by MedEqualiTeam (a French medical NGO). The results were analyzed, and relevant statistics provided to Médecins Sans Frontières, the UNHCR and MedEqualiTeam.

Results: 500 patients completed the survey (63.1\% male, $36.5 \%$ female, $.04 \%$ other), with $62 \%$ aged between 18 and 30 , and with the majority coming from Afghanistan (34.3\%) or the DRC (20.8\%). The patients reported extremely poor access to basic sanitation, drinking water and nutrition. Some $73 \%$ reported diarrhoea or vomiting, 93\% reported scabies or other insect infestation in their lodging and $91 \%$ reported psychological distress.

Conclusion: The basic needs of refugees on Samos are not being met. Improved access to sanitation, drinking water, nutrition and medical care is sorely needed to alleviate some of the suffering in the camp. Given the changeable conditions on Samos, continual surveying would be of benefit to monitor the wellbeing of refugees and the efficacy of future interventions to improve conditions.

\section{Reference}

[1] Karolina Tagaris (2019). Second Greek migrant camp in flames as arrivals continue to rise. Reuters World News 15/10/19.

Shaw et al. | URNCST Journal (2020): Volume 4, Issue 3

DOI Link: https://doi.org/10.26685/urnest.182 
UNDERGRADUATE RESEARCH IN NATURAL AND CLINICAL SCIENCE AND TECHNOLOGY (URNCST) JOURNAL Read more URNCST Journal articles and submit your own today at: https://www.urncst.com

8. Neurocognitive functioning in an obese paediatric population with and without obstructive sleep apnea Tiffany Mustonen, MB, BCh, BAO student [1, 2], Giorge Voutsas, MSc student [2-4], Sherri Katz, MSc, MDCm, FRCPC [3, 5-7], Andrea Kassner, PhD [2, 4], Tanvi Naik, MHSc [2, 3], Henrietta Blinder, MSc student [6, 7], Rashi Thakur, BSc student [2, 4], Indra Narang, MEDSCI, MBBCH, FRCPCH, MD [2-4]

[1] School of Medicine, University College Dublin, Belfield, Dublin 4, Ireland

[2] Translational Medicine, Research Institute, Hospital for Sick Children, Toronto, ON, Canada

[3] Division of Respiratory Medicine, Hospital for Sick Children, Toronto, ON, Canada

[4] University of Toronto, Toronto, ON, Canada

[5] Department of Paediatrics, Children's Hospital of Eastern Ontario, Ottawa, ON, Canada

[6] Children's Hospital of Eastern Ontario Research Institute, Ottawa, ON, Canada

[7] University of Ottawa, Ottawa, ON, Canada

Introduction: Obstructive sleep apnea (OSA) is associated with deficits in neurocognitive functioning in otherwise healthy children. However, there is little data describing neurocognition in children with OSA. The objective of this study was to compare neurocognitive functioning in children with obesity, with and without OSA.

Methods: This was a prospective study of children 8-18 years old with obesity referred for a baseline polysomnogram. All subjects completed the Conners-3 Self-Report Short and the Behavior Rating Inventory of Executive Function questionnaires. Responses were analysed for differences between subjects with and without OSA. OSA was defined as an overall obstructive apnea-hypopnea index $(\mathrm{OAHI}) \geq 5$. T-scores $>65$ were considered clinically significant and abnormal.

Results: Of the 97 children included, 41 had OSA ( $73 \%$ male, mean age $14.1 \pm 2.3$ years, mean BMI $42.4 \pm 9.5 \mathrm{~kg} / \mathrm{m}^{2}$, mean OAHI 23.6 \pm 28.6 ) while 56 did not have OSA ( $41 \%$ male, mean age $13.6 \pm 2.7$ years, mean BMI $35.7 \pm 8.9 \mathrm{~kg} / \mathrm{m}^{2}$, mean OAHI $1.5 \pm 1.4)$. Gender $(p=0.003)$, BMI $(p=0.002)$, and OAHI $(p<0.001)$ were significantly different between groups. No significant differences were found in mean T-scores, or the frequency of abnormal T-scores in children with or without OSA.

Conclusion: Our study of children with obesity found no significant differences in neurocognition scores between those with and without OSA. However, $63 \%$ of the children displayed abnormal T-scores, indicating adverse neurocognitive function. Future research is needed to identify children with obesity at highest risk for neurocognitive deficits. Acknowledgements: This research was funded by the Canadian Sleep and Circadian Network and the Heart and Stroke Foundation of Canada.

9. Vitamin D status in children with cow's milk protein allergy Sumitha G. Pandiaraja, MB, BCh, BAO Student [1], Ioana Maris, PhD [2]

[1] University College Cork, Cork, Ireland T12 K8AF

[2] Bon Secours Hospital Cork, Department of Paediatrics and Child Health, University College Cork, Ireland

Introduction: Cow's milk protein allergy (CMPA) affects 1.9-4.9\% [1] of children worldwide and can be either IgE-mediated or non-IgE mediated. Studies have highlighted the increased risk of inadequate Vitamin D intake in children with CMPA on elimination diets [2], with long-term effects on bone health, respiratory health and immune function. This study aims to investigate the risk of Vitamin D inadequacy in children with CMPA and assess their compliance to Irish Vitamin D supplementation (VDS) recommendations.

Methods: A cross-sectional chart review of 38 children with CMPA, aged 6 to 24 months, attending a paediatric allergy clinic was performed. The serum Vitamin D levels of children with CMPA $(n=15)$ were compared to non-allergic controls $(n=26)$. In addition, an online questionnaire was administered to caregivers of children with CMPA to assess compliance to VDS.

Results: The serum Vitamin D level among children with CMPA (median=48 nmol/L) was observed to be lower than that of healthy controls (median=62 nmol/L) $(\mathrm{U}=132.0, \mathrm{p}=0.088)$. However, among children with CMPA, those compliant with VDS had a significantly higher serum Vitamin D level (median $=55 \mathrm{nmol} / \mathrm{L}$ ) than those with irregular or no VDS use $($ median $=39 \mathrm{nmol} / \mathrm{L})(\mathrm{U}=8.0, \mathrm{p}=0.021)$. The online questionnaire revealed that only $38.9 \%$ of caregivers adhered fully to the recommended duration and frequency of vitamin D supplementation, despite being aware of Ireland's Health Service Executive guidelines $(94.4 \%)$.

Conclusion: Children with CMPA may be at higher risk of Vitamin D inadequacy. However, ensuring compliance to current VDS recommendations can yield higher serum Vitamin D levels in this group. 
UNDERGRADUATE RESEARCH IN NATURAL AND CLINICAL SCIENCE AND TECHNOLOGY (URNCST) JOURNAL

Read more URNCST Journal articles and submit your own today at: https://www.urncst.com

\section{References}

[1] Venter C, Brown T, Meyer R, Walsh J, Shah N, Nowak-Węgrzyn A, et al. Better recognition, diagnosis and management of non-IgE-mediated cow's milk allergy in infancy: iMAP-an international interpretation of the MAP (Milk Allergy in Primary Care) guideline. Clin Transl Allergy. 2017 Aug 23; 7(26), eCollection 2017. https://doi.org/10.1186/s13601-017-0162-y

[2] Giovannini M, D'Auria E, Caffarelli C, Verduci E, Barberi S, Indinnimeo L, et al. Nutritional management and follow up of infants and children with food allergy: Italian Society of Pediatric Nutrition/Italian Society of Pediatric Allergy and Immunology Task Force Position Statement. Ital J Pediatr. 2014 Jan 3; 40: 1. https://doi.org/10.1186/1824-7288-40-1

\section{Analysis of nuclear architecture in lobular breast carcinoma}

Tharsika Dasnamurthy Reddiar, MB, BCh, BAO (Hons) student [1] Karolina Salciute, BSc (Hons), PhD student [2], Diarmuid O'Connor, MB, BCh, BAO (Hons), PG Dip, MSc [4], Andrew Flaus, PhD [3],

Caroline Brodie, BSc, MB, BCh, BAO (Hons), MRCPath [4], Helen Dodson, PhD [2]

[1] School of Medicine, NUI Galway, Ireland

[2] Anatomy, School of Medicine, NUI Galway, Ireland

[3] Centre for Chromosome Biology, Biochemistry and School of Natural Sciences, NUI Galway

[4] Department of Anatomic Pathology, Saolta University Health Care Group, University Hospital Galway

Introduction: Lobular carcinoma is a subtype of breast carcinoma composed of relatively uniform non-cohesive cells which are individually dispersed or arranged in single files. This study aims to analyze the nuclear architecture of lobular breast carcinoma to better understand important changes in chromatin condensation and nuclear morphology as the pathology progresses.

Methods: Ethical approval was granted for analysis of 10 anonymized lobular breast carcinoma samples. Following staining with Hematoxylin and Eosin (H\&E) and microscopic imaging, the nuclei were classified into 3 groups by appearance of the chromatin organization, in particular at the nuclear periphery. The nuclei were classified according to increasing levels of atypia. The primary characteristic used to classify atypia was non-uniform chromatin condensation. Using ImageJ, area, perimeter and circularity were measured for 20 nuclei in each of the 3 groups and then compared using a Kruskal-Wallis ANOVA test.

Results: Morphological differences were evident comparing the three classes. To establish a numerical parameter for chromatin condensation, the H\&E images were converted to greyscale and the intensity of each pixel was determined. Statistically significant morphological differences were detected between the three classes for area $(\mathrm{p}<0.0001)$, perimeter $(\mathrm{p}<0.0001)$ and circularity $(\mathrm{p}=0.0009)$.

Conclusion: This preliminary study quantifies changes in chromatin organization and nuclear morphology in lobular carcinoma. By combining classification and measurements of nuclear parameters derived from molecular biology coupled with the knowledge of a highly skilled pathologist, this research demonstrates the potential for developing automated image analysis, a digital pathology tool of the future.

\section{Assessing the usefulness of three-dimensional imaging and virtual reality in surgical planning: A medical student led pilot study Clodagh Ryan, MB, BCh, BAO Student [1], Eoin O'Malley, Radiology Registrar [2], Declan Sheppard, Radiology} Consultant [1, 2]

[1] School of Medicine, NUI Galway, Ireland

[2] Department of Radiology, University Hospital Galway, Ireland

Introduction: Nephron-sparing surgery is becoming more common as surgical techniques advance. Virtual reality (VR) and three-dimensional (3D) visualisation appear to provide better anatomical understanding in pre surgical planning than twodimensional alone. 3D models may enable greater tissue salvation and fewer complications. 3D model preparation and advancing research is expensive and time consuming. We aim to pilot study led by medical student, create reliable anatomical kidney models and assess usefulness in surgical planning.

Methods: Routine computerised tomography (CT) urograms were performed on 128 slice scanner using split bolus technique. Medical student segmented and displayed models in VR using 3DSlicer. Radiology registrar and consultant validated models. Two urology surgeons completed qualitative questionnaires. 
UNDERGRADUATE RESEARCH IN NATURAL AND CLINICAL SCIENCE AND TECHNOLOGY (URNCST) JOURNAL Read more URNCST Journal articles and submit your own today at: https://www.urncst.com

Results: We included two patients. Only minor segmentation tweaks by radiologist ensured accurately demonstrated tumors. Tissue contrast quality varied between CT scans complicating segmentation. Both surgeons deemed models helpful in visualising hilar anatomy, predicting bleeding complications, determining laparoscopic/open and partial/full nephrectomy approach. Surgeons prioritised vasculature visualisation over collecting system. Surgeons suggested gauging tumor depth would be especially useful. Considering 3D printing cost, surgeons agreed VR alone may suffice.

Conclusion: Surgeons found 3D and VR enabled accurate surgical planning and patient counselling regarding nephrectomy risk. Minor CT protocol recommendations enable easier and more accurate segmentation, without increasing patient's radiation exposure. Annual leave during 8-week summer project reflects case numbers. Since, we've identified more cases to assess surgical parameters against matched cohort. We've begun work for adrenal surgery. It's feasible for medical students with minimal surgical/radiological knowledge to advance this research, gaining valuable experience.

\section{Investigating ctenophore colloblast adhesive toxicity on prey Artemia salina}

Sana Al Buraiky, MB, BCh, BAO Student [1], George O. T. Merces, BSc, MSc [1, 2], Mark Pickering, BA, PhD, [1, 2]

[1] School of Medicine, University College Dublin, Belfield, Dublin, Ireland

[2] UCD Centre for Biomedical Engineering, University College Dublin, Belfield, Dublin, Ireland

Introduction: In recent years, there has been an increased use of bio-adhesives in surgery, however, there remains a gap for the development of new alternatives. Marine bio-adhesives have the advantage of underwater efficacy, making their utilisation in vivo an area of interest. Ctenophores possess isolatable colloblast cells that secrete such adhesive, but it is important to establish its toxicity before consideration for clinical use. It has been suggested to be toxic for its role in prey capture, this study aims to investigate this hypothesis. Artemia salina are an example of ctenophores' prey, and often used as a preliminary measure for toxicity.

Methods: The adhesive was collected from ctenophores, Pleurobrachia pileus, prior to the experiment and frozen. 53 Artemia salina nauplii were used. The specimens were divided into three groups: control (in seawater), and two groups submersed in homogenised and non-homogenised adhesive, respectively. Infrared transillumination was used, while temperature and light sources were controlled for due to the animals' responsiveness to both. Light microscopy was used to visualise the motility and mortality post-exposure to the adhesive during 30 minutes.

Results: At 30 minutes, the survival was $100 \%$ in all groups. 8 of 35 in the test groups were immobilised, with limb movements still evident. This was attributed to the adhesive's viscosity, as opposed to a toxic effect.

Conclusion: There was no evidence of toxicity of the adhesive on Artemia. While the lack of evidence for toxicity is promising for clinical application, this will need to be confirmed on human cells.

\section{Documentation of pain assessment for patients receiving palliative care: A case study of Hospice Africa Uganda Susie Phelan MB, BCh, BAO Student [1], Agasha Birungi D [2] \\ [1] School of Medicine, University College Dublin, Belfield, Dublin 4, Ireland \\ [2] Clinical Department, Hospice Africa Uganda, Makindye Division, Kampala, Uganda}

Introduction: Hospice Africa Uganda (HAU) operates a palliative care service that offers regular pain assessments, and pain relief to patients. This cross-sectional study aimed to determine the percentage of patients assessed for pain at initial, second, and third consultations; to identify the average progression/regression of pain scores following their initial consultation; to identify the average change in pain scores; and to determine the percentage of patients who received three consecutive pain assessments.

Methods: The study sample consisted of 64 patient records from patients admitted to HAU during 2018. Data was extracted using Excel, a descriptive analysis was performed using SPSS Statistics Software.

Results: The study revealed that $21.3 \%$ patients received three consecutive pain assessments. $90.6 \%$ of patients were assessed for pain on their first consultation, $56.3 \%$ of patients were assessed on their second consultation, and $25 \%$ on their third consultation. A Kruskal-Wallis test revealed an insignificant difference of mean pain scores [ $p>.05]$; with a decrease in mean pain scores from 3.22 at the first consultation to 2.13 at the third consultation. Data analysis displayed that $34.3 \%$ of patients reported no change in pain scores, $51.5 \%$ of patient reported regression in pain scores, and $14.4 \%$ reported progression in pain scores.

Conclusion: The study suggests that there is a decrease in the documentation of pain assessments between a patient's first and third consultation. Furthermore, the results of the study display that current practices are not effective in decreasing patients' reported pain scores. By improving compliance to documentation, patients' pain scores can potentially be reduced between consultations. 
UNDERGRADUATE RESEARCH IN NATURAL AND CLINICAL SCIENCE AND TECHNOLOGY (URNCST) JOURNAL Read more URNCST Journal articles and submit your own today at: https://www.urncst.com

\section{The effect of bariatric surgery on diabetes: A research study}

Sarah Cavallari, BSc Student [1], Maeve Durkan, Consultant Endocrinologist [2], Colm O'Boyle, General and Bariatric Surgeon [3]

[1] School of Medicine, University College Cork, Cork, Ireland

[2] Department of Diabetes, Endocrinology and Metabolism, The Cork Clinic, Cork, Ireland

[3] Department of General and Obesity Surgery, The Cork Clinic, Cork, Ireland

Introduction: Type 2 Diabetes Mellitus has long been regarded as a chronic illness, and current standards of care aimed at slowing progression and alleviating symptoms. Though surgical intervention in the form of bariatric surgery for patients with morbid obesity has been shown to drastically improve, and reverse T2DM, it is not first line for any patient group [1]. Preoperative parameters may identify groups for whom early surgical intervention may be favourable.

Methods: Pre and post-operative data from diabetic, obese patients who underwent bariatric surgery was retrospectively analysed. Diabetic outcomes were assessed using HbA1c preoperatively, and at 36-month follow-up appointment. Data on demographics, duration of diabetes, medications and weight was also collected.

Results: Data was collected from 57 patients; 25 female, 22 male. A mean reduction in HbA1c of $18.8596 \mathrm{mmol} / \mathrm{mol}$ (range, -5-63) was shown between patients' preoperative levels and 36-month postoperative levels. 68\% (30 patients) of the group who received medication preoperatively stopped all medications by 36 months postoperatively. 6 patients were insulin dependent preoperatively, and postoperatively 3 ceased all diabetic medications, 2 achieved control with oral hypoglycaemics, 1 remained on insulin. Weight reduction and duration of diabetes showed correlation with change in diabetes status which did not achieve statistical significance.

Conclusion: Bariatric surgery is an effective method of drastic improvement and resolution of T2DM, at least at 36 months postoperatively. Duration of diabetes and severity of disease may prompt earlier surgical intervention than current standards of care, however a larger data set is required to accurately identify these patient groups.

\section{Reference}

[1] Rubino F; Nathan D; Eckel RH; Schauer PR; Alberti KG; Zimmet PZ; Del Prato S; Ji L; Sadikot SM; Herman WH; et al. Metabolic surgery in the treatment algorithm for type 2 diabetes: A joint statement by International Diabetes Organizations. Diabetes Care. 2016 Jun; 39(6): 861-77. https://doi.org/10.2337/dc16-0236

15. Does a history of concussion affect the dual-tasking ability of schoolboy rugby players? Oisin Fearon, BSc (Hons)/MB, BCh, BAO Student [1], Pooler. Archbold, MB, BCh, BAO (Hons), MCRS, MD [2], Mark Matthews BSc, MSc, PhD, Lecturer in Sport Physiotherapy, School of Sport [3], Richard Davies, BEng (Hons), PhD candidate, Lecturer in Computing Science, School of Computing [4], Chris Bleakley BSc (Hons), MCSP, SRP, PhD, Lecturer in Physiotherapy, School of Health Sciences [3] Michael Corless BSc (Hons)/MB, BCh, BAO Student [1], [1] Department of Medicine, Queen's University Belfast, Belfast, Co. Antrim BT7 1NN, UK

[2] Royal Victoria Hospital, Belfast, Co. Antrim BT12 6BA, UK

[3] Department of Sport and Exercise Sciences Research Institute, University of Ulster, Shore road, Newtownadbbey, Co. Antrim BT37 OQB, UK

[4] Department of Computer Science Research Institute, University of Ulster, Shore road, Newtownadbbey, Co. Antrim $B T 370 Q B, U K$

Introduction: Irish schoolboy rugby players face an estimated 28\% risk of injury per season, a higher risk than professional players. Concussion is the most common with $26 \%$ of all injuries sustained. Concussion causes impairments of unclear duration in motor and cognitive functions. Functions essential to play rugby. Injury incidence also increases post concussion. This study investigated if a history of concussion reduced players' ability to 'dual-task' motor and cognitive functions, potentially explaining the increased injury incidence post concussion.

Methods: 96 schoolboy rugby players aged 14-15 completed concussion history questionnaires. Each player completed both a postural stability test and 3 metre timed walk with simultaneous mental tasks to test their dual-tasking ability. Errors such as losing balance were counted. Testers were blinded to players' concussion history.

Results: $37.5 \%$ of players reported a previous concussion. While previously concussed players made more errors in the single leg balance test, the difference was statistically insignificant $(\mathrm{P}>0.05)$. With the 3 metre walk there were also no statistically significant differences between times or errors $(\mathrm{P}>0.05)$. 
UNDERGRADUATE RESEARCH IN NATURAL AND CLINICAL SCIENCE AND TECHNOLOGY (URNCST) JOURNAL Read more URNCST Journal articles and submit your own today at: https://www.urncst.com

Conclusion: The unprecedented incidence of previous concussion raises concerns given concussion's risk of death and neurodegenerative diseases. However no link between dual-tasking and previous concussion was demonstrated. Study limitations included variance in testers' error interpretation. Further work using computerised inertial sensors and the new evidence backed "Y-balance test" is currently underway to address these issues. Hopefully by improving our knowledge of concussion and its effects we can help safeguard players exposed to it.

16. Can visual function tests act as early functional biomarkers of diabetic retinopathy prior to clinical features? Constantinos Nicolaou, MB, BCh, BAO student [1] Radha Das, MS, FRCS, PhD [1] Jennifer Perais, Ms [1] Katie W. Graham, BSc (Hons) Optometry, PhD [1] Timos Naska, PhD [1] David M. Wright, PhD [1] Frank Kee, MD, PhD [1] Tunde Peto, MD, PhD [1] Nicola B. Quinn, PhD [1] Usha Chakravarthy, FRCOpth, PhD [1] Ruth E. Hogg, BSc (Hons), PhD [1]

[1] Centre for Public Health, Queens University, Belfast, United Kingdom

Introduction: The purpose of the paper is to explore whether measures of vision can differentiate age-matched controls from between (i) pre-diabetics (ii) persons with Diabetes Mellitus (DM) without Diabetic Retinopathy (DR) (iii) those with manifest DR without diabetic macular oedema (DME).

Methods: 873 participants recruited: 496 population-based controls, 103 pre-diabetics (HbA1c 6.0-6.4\%), 98 with DM (HbA1c> 6.4\% or self-report) with no evidence of DR and 176 with mild to moderate DR without DME based on ultrawidefield imaging and central macular optical coherence tomography. Best-corrected visual acuity (BCVA), low luminance acuity (LLA Distance), low luminance deficit (LLD), near visual acuity (NVA), LLA Near (SKILL Dark side), LLD for near (SKILL score) and contrast sensitivity (CS) were assessed in both eyes. MAIA microperimetry and frequency doubling threshold (FDT) perimetry were performed on the selected study eye.

GEE logistic models corrected for age and sex for all measures of vision [BCVA, LLA, LLD, NVA, CS, SKILL score, FDT mean deviation (MD), MAIA mean sensitivity (MS) and MAIA central mean sensitivity (CMS)] compared (a) controls vs DM without DR and (b) DM without DR vs DR.

Results: While none of the measure of vision distinguished between pre-diabetes and controls, several measures of vision distinguished between controls and those with DM but no DR including SKILL Dark side $(\beta=-4.80$, $\mathrm{p}<0.001)$, reading index $\beta=-11.4, p=0.002$, reading speed $\beta=2.61 \mathrm{p}=0.019)$ and FDT mean deviation $($ Beta $=-2.54 \mathrm{p}<0.001)$.

Conclusion: Visual function impairment exists in patients with DM before detectable vasculopathy and suggests the presence of neural dysfunction.

17. A quality of life study in a group of patients with hereditary angioedema residing in south-west Ireland Ellen G. Ahern, BSc Student [1], Paula O'Leary, MD, FRCPI, FRCPath [2, 3]

[1] School of Medicine, University College, Cork, Ireland T12 AK54

[2] Department and School of Medicine, University College, Cork, Munster, Ireland T12 AK54

[3] Department of Immunology, Cork University Hospital, Cork, Munster, Ireland T12 DC4A

Introduction: Hereditary Angioedema (HAE) is an autosomal dominant genetic disorder caused by a deficiency in C1 inhibitor, characterised by recurrent attacks of swelling [1]. Attacks interfere with daily life, causing embarrassment, discomfort and pain. Laryngeal swelling is potentially life-threatening [2]. This study aims to identify specific and relevant information regarding the precise impact of disease on quality of life (QoL).

Methods: This is a descriptive, cross-sectional study. Convenience sampling was used to recruit patients attending the specialist in CUH. QoL was evaluated using the AE-QoL validated questionnaire, via postal survey packet. Data was analysed under 4 headings; functioning, fatigue/mood, fears/shame and nutrition. A total score for each patient and overall mean was calculated. Results: Of the data collected from 9 patients, 5 had total scores greater than $50 \%$ in their self-assessed AE-QoL. Three scored greater than $61 \%$, with one scoring $78 \%$. Only one participant scored $0 \%$. A $50 \%$ score indicates a moderate impact on QoL, with scores above $70 \%$ indicating great adverse impact. Of the 4 domains evaluated, on average, fatigue/mood reported the highest impact on QoL (46.67\% mean). Functioning was the domain with the least reported impact on QoL, with a mean score 31.94\%.

Conclusion: HAE is a serious condition with significant impact on QoL. Patients report a relatively high disease burden, with significant effect on their energy levels and mood, causing considerable problems with fatigue and depression. Use of this questionnaire has exposed areas of importance for patients which might not otherwise come to light in standard clinical consultation settings.

Shaw et al. | URNCST Journal (2020): Volume 4, Issue 3

Page A9 of A46

DOI Link: https://doi.org/10.26685/urncst.182 
UNDERGRADUATE RESEARCH IN NATURAL AND CLINICAL SCIENCE AND TECHNOLOGY (URNCST) JOURNAL

Read more URNCST Journal articles and submit your own today at: https://www.urncst.com

\section{References}

[1] Donaldson VH, Evans RR. A biochemical abnormality in hereditary angioneurotic edema. Absence of serum inhibitor of C'1-esterase. Am J Med. 1963 Jul; 35(1): 37-44. https://doi.org/10.1016/0002-9343(63)90162-1

[2] Agostoni A, Aygören-Pürsün E, Binkley KE, Blanch A, Bork K, Bouillet L, et al. Hereditary and acquired angioedema: Problems and progress: Proceedings of the third $\mathrm{C} 1$ esterase inhibitor deficiency workshop and beyond. J Allergy Clin Immunol. 2004 Sep; 114(3): S51-131. https://doi.org/10.1016/j.jaci.2004.06.047

18. Mid-term outcomes of ceramic-on-ceramic total hip arthroplasties in Cork, Ireland: A research study Danielle M. Gillette BSc [1], Kas Linehan RN [2], Lindsay A. Tetreault PhD [1], James Harty MB, MSc, MRCSI, FRCSI, (Tr\&Orth) [2, 3], Rehan Gul AFRCSI, FRCSI, FRCS (Tr\&Orth) [2, 3]

[1] Graduate Entry Medicine, University College Cork, Cork, Ireland

[2] Department Department of Orthopaedics, South Infirmary Victoria University Hospital, Cork, Ireland

[3] Department Department of Trauma and Orthopaedics, Cork University Hospital, Cork, Ireland

Introduction: We conducted a prospective study of ceramic-on-ceramic total hip arthroplasties (THA) in Cork, Ireland to determine the 5-year functional outcomes and evaluate mid-term survivorship.

Methods: Between January 2011 and December 2014, 238 hips underwent THA via an anterior (110 hips; $46.22 \%)$ or posterior approach (128 hips; 53.78\%). Only patients who received ceramic-on-ceramic implants were included. At each follow-up, clinical outcomes of WOMAC and SF-12 functional scores were recorded, and a paired t-test was used to compare pre- and post-operative scores. A Kaplan-Meier analysis was undertaken to estimate survival.

Results: The patient demographics included 147 (61.76\%) men and 91 (38.24\%) women with a mean age at surgery of 51.68 years (16-74). The mean pre-operative WOMAC score of 38.70 improved to 87.93 ( $\mathrm{p}<0.0001$ ), while the SF-12 score of 29.82 improved to 31.61 ( $\mathrm{p}<0.0001$ ), at final follow-up. The five-year survivorship without re-operation was $98.32 \%$ (4/238 were revised).

Conclusion: These findings demonstrate that surgery significantly improved functional scores and that rates of surgical revision of THA in Cork are low. This is comparable to rates found in Australia, who benefit from a national joint registry which provides evidence-based guidelines. It is shown that the current practice in Cork is aligned with best practice in Australia. It is therefore suggested that once Ireland's national joint registry begins to output data analysis, the wealth of information could be used to influence guidelines and allow for future research to compare outcomes in a global setting.

19. Mesenchymal stem cell-derived extracellular vesicles for the treatment of acute respiratory distress syndrome: Concise review

Aswin Abraham, MB, BCh, BAO Student [1], Anna Krasnodembskaya, PhD [2]

[1] School of Medicine, Dentistry and Biomedical Sciences, Queen's University, Belfast, Northern Ireland, BT9 7BL United Kingdom

[2] Wellcome-Wolfson Institute for Experimental Medicine - School of Medicine, Dentistry and Biomedical Sciences, Queen's University, Belfast, Northern Ireland, BT9 7BL United Kingdom

Introduction: Acute respiratory distress syndrome (ARDS) is a potentially fatal acute inflammatory lung condition affecting approximately 3 million people each year, with a 32\% 60-day mortality rate. Despite this, ARDS has no pharmacological treatments. However, mesenchymal stem cells (MSCs) have shown very promising therapeutic potential, and recently, it was established that their effect is largely due to the transfer of MSC derived extracellular vesicles (MSC EVs). The review discusses preclinical studies investigating the therapeutic potential of MSC EVs in ARDS.

Methods: We conducted a review of 12 papers found using PubMed search. Search criteria included papers published between 2010 and 2019 involving the use of MSC EVs in the treatment of either ARDS or acute lung injury (ALI) either within in-vivo or ex-vivo models.

Results: MSC EVs transfer substances such as mRNA, miRNA, and mitochondria to ameliorate ARDS in preclinical models. Molecularly, they reduce inflammatory cell influx into alveoli, decrease levels of proinflammatory cytokines such as IL-8, TNF-, MIP-2, IL-1 ${ }^{\circledR}$, IL-6 and IL-17 and increase levels of anti-inflammatory cytokines such as IL-10. Macroscopic effects include an increase in alveolar fluid clearance, reduction in lung weight gain and decrease in pulmonary artery pressure and resistance. Importantly, they have been proven to have the same effect as their parent cells.

Conclusion: MSC-derived EVs improve the causal factors of ARDS in preclinical conditions, both in vivo and ex vivo. Further research into their mechanism of action, biodistribution, standardisation and development of better manufacturing techniques is required before translation to clinical studies [1].

Shaw et al. | URNCST Journal (2020): Volume 4, Issue 3

DOI Link: https://doi.org/10.26685/urnest.182 
UNDERGRADUATE RESEARCH IN NATURAL AND CLINICAL SCIENCE AND TECHNOLOGY (URNCST) JOURNAL Read more URNCST Journal articles and submit your own today at: https://www.urncst.com

\title{
Reference
}

[1] Abraham A, Krasnodembskaya A. Mesenchymal stem cell-derived extracellular vesicles for the treatment of acute respiratory distress syndrome: Concise Review. Stem Cells Transl Med. 2020 Jan; 9(1): 28-38. https://doi.org/10.1002/sctm.19-0205

\author{
20. Spreading the word: A participatory approach to developing evidence-based education and awareness resources \\ for the rare disease inflammatory arthritis of down syndrome \\ Amirah. Alzaki, MBBC Student [1], Aisling. McNiffe [2], Andre. Valente, B.Sc, PhD [3] and Emma. Dorris, PhD [4] \\ [1] UCD School of Medicine, University College Dublin, Belfield, Dublin 4, Ireland \\ [2] The Patient Voice in Arthritis Research, hosted by the UCD Centre for Arthritis Research, Belfield, Dublin 4, Ireland \\ [3] Champalimaud Foundation, Lisbon, Portugal \\ [4] UCD Centre for Arthritis Research, School of Medicine, University College Dublin, Belfield, Dublin 4, Ireland
}

Introduction: Children with Down syndrome (DS) are at increased risk of developing inflammatory arthritis. Inflammatory arthritis of DS (IADS) is a clinically distinct form of arthritis with a higher prevalence rate (20 per 1000) compared to the rate of juvenile idiopathic arthritis within the general population (1 per 1000) [1, 2]. IADS is often under-reported and diagnosis is often greatly delayed. As a result, children with IADS present with significant joint damage and disability at diagnosis [1]. The aim of this research was to increase knowledge translation of the novel findings of recent research to the gatekeepers of early intervention: primary caregivers, carers, parents and people with DS.

Methods: We worked with two parents of children with IADS to discuss challenges and identify which communication tools would be most useful to raise awareness of those key challenges of IADS. Involvement partners were part of the project from conception to dissemination. In response, a video and an information brochure were designed. For both resources, we used an iterative design process with multiple review rounds from both the target audiences and the clinical professionals.

Results: Production of an information animation video designed for social media circulation, primarily aimed at people with and carers of those at risk of IADS. Also, we created an information brochure targeted to primary care professionals, patients, their families, tertiary care professionals who do not know IADS well, the R\&D community and the general population.

Conclusion: Overall, parents greatly favoured an easily sharable resource (the video) and a visual brochure with easy to remember facts.

\section{References}

[1] Foley C, Killeen O. Musculoskeletal anomalies in children with Down syndrome: an observational study. Archives of Disease in Childhood. 2018; 104(5): 482-487. http://dx.doi.org/10.1136/archdischild-2018-315751

[2] Foley C, Deely D, MacDermott E, Killeen O. Arthropathy of Down syndrome: an under-diagnosed inflammatory joint disease that warrants a name change. RMD Open. 2019; 5(1): e000890. https://doi.org/10.1136/rmdopen-2018-000890

\author{
21. An exploration of the expectations of a new Primary Care Centre in North Cork city \\ F. Cooney, BSc Student [1], WH. Smithson, MD [2], M. Kugadas, PhD [3] \\ [1] School of Medicine, University College Cork, Cork, Ireland \\ [2] Department of General Practice, University College Cork, Cork, Ireland \\ [3] Department of Medicine, Bons Secours Hospital, Cork, Ireland
}

Introduction: 140 primary care centres (PCC) have become operational in Ireland, between 2001 and 2019 [1]. These new facilities aim to make health services easily accessible, integrated and locally based [2]. This is a qualitative study of a community's expectations and knowledge of a new primary care centre that is being built in their area.

Methods: A total of three semi-structured focus groups were carried out ( 20 participants in total) in NICHE community centre, with people from the local community. Written consent was obtained from all participants prior to taking part. All focus groups were audio-recorded and then transcribed verbatim. Thematic analysis was then carried out, identifying themes and sub-themes. Results: Several issues were highlighted by participants. The types of services that will be available in the PCC and the services participants hoped to be in the centre. Current issues, such as waiting times and access to services were discussed, which participants hope will improve with the opening of the PCC. Participants also stressed the importance of community involvement and information sharing. They also expressed their own fears and concerns with regards to the new facility.

Conclusion: The findings indicate poor community involvement and poor provision of information leading to concern about the size and speed of the build and whether this is good use of public money. Many incorrect assumptions have been made about planned services and the focus groups were seen as information gathering sessions. Engagement would limit those misassumptions and lead to better use of the centre. 
UNDERGRADUATE RESEARCH IN NATURAL AND CLINICAL SCIENCE AND TECHNOLOGY (URNCST) JOURNAL

Read more URNCST Journal articles and submit your own today at: https://www.urncst.com

\section{References}

[1] Mercille, J. The Public-Private Mix in Primary Care Development: The Case of Ireland. Int J Health Serv. 2019 Jul; 49(3): 412-430. https://doi.org/10.1177/0020731419836079

[2] Houses of the Oireachtas Committee on the Future of Healthcare. Sláintecare Report. May 2017.

22. Bariatric surgery improves cardiovascular risk in the early postoperative period Aaron Kwun Hang Ho, MB, BCh, BAO Student [1], Colm J O'Boyle, MB, BCh, BAO [2], Hayder Shabana, MD [2] [1] School of Medicine, University College Cork, Cork, Ireland [2] Department of Surgery, Bons Secours Hospital, Cork, Ireland

Introduction: The effect of bariatric surgery (BS) on improvement in hypertension is well documented. Far-less is known regarding the timing and duration of this improvement and the concurrent effect on antihypertensive usage.

Methods: This study was a retrospective review of 618 patients undergoing primary laparoscopic sleeve gastrectomy (LSG) and gastric bypass (LGBYP) under the care of a single Consultant Surgeon between January-2009 and June-2019. Weight, height, BMI, systolic blood pressure (SBP), diastolic blood pressure (DBP) and antihypertensive usage were recorded preoperatively and postoperatively at 1-month and 1-year. Statistical analysis was performed using SPSS and p<0.05 was considered significant.

Results: $468(76 \%)$ patients were female. The mean age was $46 \pm 11$ years and the mean BMI was $49 \pm 8 \mathrm{~kg} / \mathrm{m}^{2} .423(68 \%)$ underwent LGBYP and the remainder underwent LSG. Postoperatively, the mean BMI fell to $43 \pm 7 \mathrm{~kg} / \mathrm{m}^{2}$ and $33 \pm 77 \mathrm{~kg} / \mathrm{m}^{2}$ at 1 -month and 1-year respectively $(\mathrm{p}<0.001$, t-test). The mean SBP reduced from $140 \pm 18 \mathrm{mmHg}$ preoperatively to $129 \pm 17$ $\mathrm{mmHg}$ and $127 \pm 18 \mathrm{mmHg}$ at 1 -month and 1 -year postoperatively respectively $(\mathrm{p}<0.001, \mathrm{t}$-test). The mean DBP decreased from $87 \pm 10 \mathrm{mmHg}$ preoperatively to $83 \pm 10 \mathrm{mmHg}$ and $72 \pm 24 \mathrm{mmHg}$ at 1 -month and 1 -year postoperatively respectively $(\mathrm{p}<0.001$, t-test). $125(52 \%)$ patients were normotensive and stopped all antihypertensives by 1-year postoperatively. Of patients taking only one or two antihypertensives preoperatively, $100(60 \%)$ were normotensive and stopped all antihypertensives by 1-year postoperatively.

Conclusion: Bariatric surgery for morbidly obese patients improves cardiovascular risk from early in the postoperative period. The improvement in blood pressure parameters occurs in conjunction with a reduction in the need for antihypertensive usage.

\section{The effect of anxiety and depression on the perception of heart failure symptoms: A research study [JN1] Hafsa B. Ali, BSc Student [JN2] [1] \\ [1] Institute of Psychology, Psychiatry and Neurosciences, King's College London, London, Greater London, England, SE5 8AF}

Introduction: Heart failure is a chronic debilitating condition that often presents with comorbid anxiety and depression, management of which can improve psychological outcomes and perception of heart failure symptoms. However, gender differences in the presentation of anxiety and depression may moderate the efficaciousness of indicated treatments. Therefore, this study aims to assess the correlation between anxiety and depression to heart failure symptom perception, and whether this is moderated by gender.

Methods: This was a cross sectional observational study scoring 61 participants on the Hospital Anxiety and Depression Scale and the revised Illness Perception Questionnaire via telephone interviews. Anxiety and depression were regressed against heart failure symptom perception, with an independent samples t- Test conducted to detect relevant gender differences.

Results: A significant positive correlation was demonstrated between the number of typical heart failure symptoms reported with anxiety $(\mathrm{t}=2.38, \mathrm{p}=0.02)$ and depression $(\mathrm{t}=2.79, \mathrm{p}<0.01)$. The proportion of reported symptoms being personally attributed to heart failure also followed the same pattern, and this was seen to greater effect in females $(t=-2.10, p=0.04)$.

Conclusion: In those who were anxious, females reported more typical symptoms of heart failure, with the gender discrepancy amongst depressed persons being more variable. Correct identification and treatment of psychiatric co-morbidities is difficult to achieve in the entire heart failure population, therefore it may be of benefit to focus energies in identifying and offering more focused treatment to anxious females, who may show more efficacious gains from psychological interventions. 
UNDERGRADUATE RESEARCH IN NATURAL AND CLINICAL SCIENCE AND TECHNOLOGY (URNCST) JOURNAL Read more URNCST Journal articles and submit your own today at: https://www.urncst.com

\author{
24. A study comparing the angiogenic properties of CD362+ Mesenchymal Stromal Cells derived from Bone Marrow \\ and Umbilical Cord \\ Ahmed Eltayeb, MB, BCh, BAO Student [1], Michael Creane, BSc, MSc, PhD [2], Timothy O'Brien, MB, BCh, BAO, PhD [2, 3] \\ [1] School of Medicine, NUI Galway, Galway City, Co. Galway, Ireland, H91 V4AY \\ [2] Regenerative Medicine Institute, NUI Galway, Galway City, Co. Galway, Ireland H91 CF50 \\ [3] University Hospital Galway, Galway City, Co. Galway, Ireland H91 YR71
}

Introduction: By 2045, Diabetes Mellitus (DM) will affect 629 million people. [1] Diabetic Foot Ulcers (DFUs) lead to amputations in 14-20\% of patients. Mesenchymal Stromal Cell (MSC) therapies target this unmet medical need. Bone Marrow-derived MSCs (BM-MSCs) promote wound-healing in DFU models in animals and humans. Umbilical Cord-MSCs (UC-MSCs) have demonstrated longer culture, higher proliferation capacity, lower senescence markers and higher Ang-1 secretion. [2]

Methods: Ethically approved UC-MSCs and BM-MSCs from healthy adults were isolated and culture expanded at Passage 3 until $>90 \%$ confluency was achieved. Conditioned Media (CM) containing BM- \& UC-MSC secretions after 24 hrs of incubation $\left(37^{\circ}, 5 \% \mathrm{CO}_{2}, 5 \% \mathrm{O}_{2}\right)$ was used in Matrigel assays to measure Human Umbilical Vein Endothelial Cell (HUVEC) tubulogenesis along with positive/negative controls $(\mathrm{N}=1, \mathrm{n}=4)$. Six images were taken at random per well after $18 \mathrm{hrs}$ and mean number of tubules was calculated. Scratch assays measured percentage closure of scratches in HUVEC monolayers, using images at time $0 \mathrm{hrs}$ and $8 \mathrm{hrs}$ of incubation, utilizing BM- \& UC-MSC-CM along with positive/negative controls (N=1, n=8). Results: UC-MSC-CM significantly increased tubulogenesis (Mean (SD) 13.50(4.44), p-value $=0.0001$ ) compared to the negative control (3.80(2.00)), while BM-MSC-CM showed no significant increase (3.87(1.42)). UC- and BM-MSC-CM significantly increased HUVEC migration (32.99(5.33) and 26.70(10.58), p value=0.0001) compared to the negative control (3.90(3.24)). No difference was observed in HUVEC migration between UC- and BM-MSC-CM (p-value=0.3652).

Conclusion: UC-MSC-CM was superior to BM-MSC-CM at inducing tubulogenesis but was no different at stimulating HUVEC migration. BM-MSC-CM demonstrated no tubulogenic effect on HUVECs but did improve migration compared to the control.

\title{
References
}

[1] IDF Diabetes Atlas. 8th edition ed. Brussels, Belgium: International Diabetes Federation 2017.

[2] Hye JJ, Yun KB, Miyeon K, Soon-Jae K, Hong BJ, Soo JC, Seong WK, Yoon SY, Wonil O, Jong WCh Comparative analysis of human mesenchymal stem cells from bone marrow, adipose tissue, and umbilical cord blood as sources of cell therapy. Int J Mol Sci. 2013 Sep 3; 14(9): 17986-8001. https://doi.org/10.3390/ijms140917986

\author{
25. Aetiology and functional outcomes following hypoxic ischaemic brain injury \\ Sian Roberts-Walsh, MB, BCh, BAO Student [1], Prasanth Sukumar, PhD Fellow [1], Valerie Twomey, PhD [2], \\ Áine Carroll, $M B, B C h, B A O, M D, F R C P, F R C P I, D L Q[1,2]$ \\ [1] UCD School of Medicine, University College Dublin, Belfield, Dublin, Ireland \\ [2] National Rehabilitation Hospital, Rochestown Avenue, Dún Laoghaire, Co. Dublin, Ireland
}

Introduction: Hypoxic ischaemic brain injury (HIBI) is a leading cause of long-term neurologic disability. This study aimed to investigate the aetiology and outcome patients with HIBI admitted to a National Rehabilitation Hospital (NRH) over a 10-year period.

Methods: Retrospective analysis of the healthcare records of all patients discharged from the NRH with an ICD-10 code for HIBI from 2000-2018. Descriptive analysis and analysis of level of disability (using Modified Barthel Index [MBI] and Disability Rating Scale [DRS]) and discharge destination was carried out using SpSS version 24.0.

Results: 572 episodes were recorded under the code g391. After exclusion of duplicate entries, the number of records analysed was 139. A further 35 were excluded, not having met the inclusion criteria. 104 records were reviewed systematically using a standardised proforma. $69(66 \%)$ were male and $35(33 \%)$ were female. Cardiovascular causes were most common (35.6\%), followed by overdose $(22.1 \%)$. Most had moderate to severe disability on admission (MBI and DRS). Severe disability was associated with respiratory arrest, overdose and neurological causes, while independence was most likely with cardiovascular causes. MBI and DRS improved in the majority (49\% and 38.5\%) with the greatest improvements seen with cardiovascular aetiology. $45.2 \%$ of patients were discharged home, $18.3 \%$ to a nursing home and $18.3 \%$ to an acute hospital.

Conclusion: Improvement in functional outcomes following HIBI is correlated to aetiology. This may have implications in helping to predict patient outcome post-HIBI. Study limitations include incomplete recordings on charts and varying sample sizes within a etiological groupings.

Shaw et al. | URNCST Journal (2020): Volume 4, Issue 3

DOI Link: https://doi.org/10.26685/urnest.182 
UNDERGRADUATE RESEARCH IN NATURAL AND CLINICAL SCIENCE AND TECHNOLOGY (URNCST) JOURNAL Read more URNCST Journal articles and submit your own today at: https://www.urncst.com

\title{
26. EGFR mutated lung adenocarcinoma and tumour staging
}

H. Abuali, BSc Student [1], A Kelly, M.B.B.S [2], M. Keane, Prof [2], C. McCarthy, M.R.C.I.,Ph [2]

[1] UCD school of Medicine, University College Dublin, Belfield, Dublin 14, Ireland

[2] Department of Medical Respiratory, St Vincent's University Hospital, Dublin 4, Ireland

Introduction: Lung cancer was responsible for 2.09 million deaths in 2018 globally. Adenocarcinoma of the lung is the most common type and accounts for $45 \%$ of all lung cancer cases [1]. Mutations in genes encoding components of Epidermal Growth Factor Receptors (EGFR) can be present and may dictate personalised therapy approaches.

Methods: The aim of this study was to determine whether there were differences in staging at diagnosis between EGFR and non-EGFR mutated adenocarcinoma. Data was extracted, for the period June 2016 to June 2019, from the National Cancer Clinical Pathway Rapid Access Lung Cancer Clinic at St Vincents' University Hospital.

Results: 730 patients were diagnosed with lung cancer over this 3-year period. 678 (93\%) of patients had non-small cell lung cancer and 46(6.3\%) were small cell lung cancer. Of 678 patients, 221 (32.5\%) were squamous cell carcinoma and 447 (61\%) were adenocarcinoma. EGFR mutations were present in $36(8 \%)$ of adenocarcinoma cases. 24\% of EGFR positive cases presented with advanced stage IV disease compared to $12 \%$ of EGFR negative cases., however this was not statistically significant (chi-square $=3.5157 . \mathrm{p}=0.318$ )

Conclusion: EGFR mutated adenocarcinoma may be associated with more advanced stage lung adenocarcinoma, however larger studies are needed to clarify this and mechanistic studies are needed to understand the pathobiology underlying this.

\section{Reference}

[1] Paul A. Bunn Jr. Worldwide Overview of the Current Status of Lung Cancer Diagnosis and Treatment. 2012. Arch Pathol Lab Med. 2012 Dec; 136(12): 1478-81. https://doi.org/10.5858/arpa.2012-0295-SA

\author{
27. Concussion in Ulster Schoolboy Rugby: Incidence, risk factors and reporting \\ Michael Corless, BSc Student Medical Sciences intercalated degree QUB [1] \\ [1] School of Medicine, Dentistry and Biomedical Sciences, Queen's University Belfast, University Road, Belfast BT7 1NN
}

Introduction: Rugby union is one of the most popular field sports in the world. Rugby became professional in 1995, which has advanced the strength and athleticism of players. Concussion is an increasingly prevalent problem in rugby union. A concussion is a traumatic brain injury, and research has shown that repeated concussions can be seriously detrimental to long term health. Concussion in schoolboy rugby is a subject that hasn't been extensively researched.

Methods: Players were recruited from 6 rugby playing schools across Ulster. A data collection system was set up online. A team of researchers travelled to the schools and the players initially completed questionnaires before key anthropometric measurements were taken. Researchers then completed a series of tests including the Y-balance test to assess both physical and cognitive capabilities.

Results: Out of the 418 total injuries reported, 114 were concussions (27.9\%). 37.9\% of players reported a history of concussion. $87.3 \%$ of players who have suffered a concussion strength train at least once per week, compared to $57.7 \%$ nonconcussed players. In the concussed player group, the number of subsequent non-concussion related injuries was 76 . This compares to 61 injuries suffered pre-concussion in this group. Second row forwards had a higher risk of concussion than any other position.

Conclusion: Rugby is an inherently physical game and head injuries are inevitable. However, this study adds to the growing body of evidence on concussion in rugby. Future work can investigate the risk factors identified in this study with a view to making the game safer for players.

28. The prognostic significance of muscle fibrillation in patients with Motor Neuron Disease Morgan J. Walsh, MB, BCh, BAO Medicine [1]

[1] School of Medicine, University College Cork, College Road, Cork, T12 K8AF, Ireland

Introduction: Motor Neuron Disease (MND) describes a group of fatal neurodegenerative diseases. Median survival time is 20-48 months however a minority of patients live greater than 10 years after diagnosis [1]. Despite a number of prognostic indicators in MND, formulation of an accurate prognosis remains an ongoing challenge.

Aim: To assess the prognostic value of the number of muscle regions at which fibrillation was detected using electromyography at initial assessment of patients with MND. 
UNDERGRADUATE RESEARCH IN NATURAL AND CLINICAL SCIENCE AND TECHNOLOGY (URNCST) JOURNAL Read more URNCST Journal articles and submit your own today at: https://www.urncst.com

Methods: A retrospective chart review was conducted of 67 patients with suspected MND assessed in the Department of Neurophysiology in Cork University Hospital (CUH) during 2006-2012. Age, initial electromyogram (EMG) results and clinical impressions were collected. Exclusion and inclusion criteria were then applied. Survival times from diagnosis were compared against the number of areas out of three distinct muscle regions at which fibrillation was detected. Data was analysed by Cox-regression and Kaplan-Meier (KM) graphs using SPSS v26.

Results: 34 patients were included in the study (21 male; 13 female). Mean survival from time of diagnosis for the entire cohort was 24.9 months (standard deviation (SD) 4.3). Mean survival was 46.5 months (SD 12.1) for 0 regions group, 28.8 months (SD 8.3) for 1 region, 13.4 months (SD 4.1) for 2 regions and 15.9 months (SD 5.9) for 3 regions. Survival rates decreased similarly on KM and Cox-regression analysis. Overall statistical result was found to be significant $(\mathrm{p}<0.011)$.

Conclusion: Patients with MND presenting with muscle fibrillation at a greater number of muscle regions on initial EMG testing had an overall decreased survival time.

29. Characteristics and outcomes of paediatric patients with chronically high EBV viral loads post renal transplant Sorcha O'Rourke, MB, BCh, BAO Student [1], Alanna Dolan MB, BCh, BAO Student [1], Samira Smith BSc [2], Michael Riordan MD [3], Maria Stack MD [3], Clodagh Sweeney MD [3], Jeff Connell PhD [2], Jaythoon Hassan PhD [2], Atif Awan MD [1, 3], Tara Raftery PhD [3].

[1] UCD School of Medicine, University College Dublin, Belfield, Dublin 4, Ireland

[2] UCD National Virus Reference Laboratory, University College Dublin, Belfield, Dublin 4, Ireland

[3] Children's Hospital Ireland, Temple Street, Dublin 1, Ireland

Introduction: Epstein Barr Virus (EBV) is a major cause of morbidity and mortality for recipients of solid organ transplantation and is related to early post-transplant lymphoproliferative disease. The study profiled EBV Loads (EBVLs), medical management and clinical outcomes in a cohort of paediatric kidney transplant recipients with chronically high EBVLs. Methods: A retrospective chart review 2005-2019 was performed. Chronically high EBVLs were defined as $>10^{5} \mathrm{copies}$ per/ml whole blood for $>6$ months in $>50 \%$ of samples tested. Statistical tests included Mann-Whitney test and correlation coefficient tests [SPSS (v24)], with a $\mathrm{p}$ value of $<0.05$ considered significant.

Results: Twenty six patients were followed up for between 3-5 years. At transplant median age was 5.7(4.09-9.47) years and 23 patients were EBV naïve. Time to activation/reactivation of the virus was 42(33-51) days, time to peak EBVL was 245(94-523) days and peak levels were $\log 5.6(5.3-6.1)$ copies/ml whole blood. 22 patients were symptomatic and 4 asymptomatic with EBV. Immunosuppression was reduced using standard protocols, two patients had acute rejection and 1 patient developed PTLD (after 7 years). IVIG $(n=4)$ and anti-viral therapy were used $(n=12)$ with no change in viral loads. Rituximab was used ( $n=2)$ and resolved the viral load for 3 months and >18 months respectively. No correlation between EBVLs and renal function was identified.

Conclusion: The clinical efficacy of antiviral therapy in this patient group is unconfirmed. Larger studies examining the efficacy of rituximab in this patient group are warranted. The current reduced immunosuppression regimen appears protective against rejection compared to higher rates reported in the literature.

\section{A study in immuno-virology techniques and application of genotype identification in the diagnosis of chronic hepatitis B infection}

Liam J. Ryan, MB, BCH, BAO Student [1], Liam Fanning, Gabriella Rizzo, PHD [1]

[1] Department of Medicine, University College Cork, Cork University Hospital, Cork Ireland

Introduction: Chronic Hepatitis B Virus (HBV) is a serious global health problem and a major cause of liver failure, liver cirrhosis and hepatocellular carcinoma. HBV is prone to mutations which has resulted in a variety of genotypes and sub-genotypes. Evidence reveals that HBV genotype is associated with differing HBV endemicity, transmission mode and clinical outcomes. The objective of this study was to understand and apply the laboratory techniques that are used in the diagnosis and classification of Chronic HBV Infection and utilising HBV genotype databases to assess the purified samples.

Methods: The methods utilised to do this included:

- Purification of IgG Antibody specific to Hepatitis B Virus.

- Isolation of HBV DNA from the serum sample.

- Amplification of the HBV DNA using Polymerase Chain Reaction.

- Confirmation the DNA is present and is HBV DNA.

- Identifying the genotype of the HBV DNA.

- Analyse the genotypes for their phylogenetics.

Shaw et al. | URNCST Journal (2020): Volume 4, Issue 3

DOI Link: https://doi.org/10.26685/urnest.182 
UNDERGRADUATE RESEARCH IN NATURAL AND CLINICAL SCIENCE AND TECHNOLOGY (URNCST) JOURNAL

Read more URNCST Journal articles and submit your own today at: https://www.urncst.com

Results: Results showed that the serum samples that were provided had been isolated successfully and were subsequently identified correctly using the methods above. Each Genotype's accession number was used to identify the genotype of HBV that was isolated and various information on the genotype was collected.

Conclusion: In conclusion, the techniques currently used for the identification of Chronic HBV genotype are effective \& efficient. The depth of knowledge on immuno-virology of Chronic Hepatitis B Infection possessed by the students was improved as a result of this study. Further analysis of how genotypes of HBV came to be present in the Irish population is required.

\title{
31. A research study on fibroblast-derived pluripotent stem cell differentiation to neural stem cells Kate J. O'Brien, MB Student [1], Timothy O'Brien, MB, BCh, BAO, PHD [1, 3], Sanbing Shen, BSc, MSc, PhD [1, 3], Jun Ma, PhD [2, 3], Huixian Cui, PhD [2, 3], Meimei Yang, PhD [1], Claire M. Coleman BSc Student [1] [1] REMEDI, National University of Ireland Galway, Galway, Connaught, Ireland \\ [2] Department of Anatomy, Hebei Medical University, Shijiazhuang, Hebei, China \\ [3] Joint Stem Cell Research Centre, National University of Ireland Galway and Hebei Medical University, Ireland
}

Introduction: Amyotrophic lateral sclerosis (ALS) is a devastating neurodegenerative disease characterized by the rapid death of upper and lower motor neurons. This degeneration leads to progressive muscle atrophy and paralysis. The disease is fatal, with most patients' death occurring within an average timeframe of 3 to 5 years, typically from respiratory failure [1] (Fujimori, Ishikawa et al. 2018). The use of neural stem cells (NSCs) may present as a novel form of cell replacement therapy to treat ALS. In this project, we differentiated induced pluripotent stem cells (iPSCs) from two sporadic ALS patients and two healthy controls into NSCs. The cell lines were then tested for appropriateness for drug screening.

Methods: The iPSCs were derived from skin fibroblasts using Sendai virus (CytoTune-iPS Sendai Reprogramming kit), from Thermofischer. NSCs were derived from human iPSCs using dual SMAD inhibition, after 7 days of neural induction. At the end of the 7 days, the cells were fixed and immunocytochemically processed using NESTIN, TUJ1, and Hoechst to confirm the identity of NSCs.

Results: The NSCs tested positive to the chosen NSC marker: NESTIN and neuronal cell marker: TUJ1. Indicating that all iPSC lines had differentiated to NSCs successfully.

Conclusion: The iPSC lines of ALS52C3, ALS53C5, ALSH84C2, and ALSH47C1 had differentiated into approximately $100 \%$ NSCs. These NSCs could be used in drug screening.

\section{Reference}

[1] Fujimori, K., et al. 2018; Modeling sporadic ALS in iPSC-derived motor neurons identifies a potential therapeutic agent. Nature Medicine. 2018; 24(10): 1579-1589. https://doi.org/10.1038/s41591-018-0140-5

\author{
32. E.coli causing de Novo Bile Duct Stone Association Revisited \\ Jeremy Kay Hock Lee, MB, BCH, BAO Student [1], Stephen Kin Yong Chang, MBBS [2, 3] \\ [1] College of Medicine and Health, University College Cork, Ireland \\ [2] GLAD Clinic, Farrer Park Hospital, Singapore \\ [3] Department of Surgery, Yong Loo Lin School of Medicine, National University of Singapore, Singapore
}

Introduction: Biliary complications remain a common source of morbidity and mortality in patients who had undergone living donor liver transplant (LDLT). However, the finding of a common bile duct (CBD) stone after LDLT is a rare occurrence. We report a case of a post-transplant patient who was admitted for gastroenteritis caused by Escherichia coli (E.coli). Three weeks later, following the acute episode, he developed a CBD stone. We hypothesize that ascending infection by E.coli in immunocompromised patients plays a role in the pathogenesis of CBD stone formation.

Methods: A single patient who developed a CBD stone following an episode of gastroenteritis and LDLT performed 5 months ago, was studied in detail. We tracked a series of his Liver Function Tests (LFTs) in the 5 month period following his LDLT. The changes in his LFTs were plotted on a graph and the results were analysed.

Results: Our patient presented with gastroenteritis with E.coli as the causative organism. He was subsequently treated with Azithromycin. Following the resolution of his gastroenteritis, his LFTs showed normalization and improvement. Total bilirubin (73 to $15 \mathrm{umol} / \mathrm{L}$ ), ALT (249 to $106 \mathrm{U} / \mathrm{L})$, AST (210 to $50 \mathrm{U} / \mathrm{L}), \mathrm{GGT}$ (900 to $221 \mathrm{U} / \mathrm{L}$ ) and ALP (163 to $84 \mathrm{U} / \mathrm{L}$ ) decreased towards near normal levels. However one month later, there was an acute exacerbation of his LFTs, which showed a marked increase in ALT (106 to $527 \mathrm{U} / \mathrm{L}$ ), AST (50 to $248 \mathrm{U} / \mathrm{L}$ ), GGT (221 to $1049 \mathrm{U} / \mathrm{L}$ ) and ALP (84 to 260U/L). A magnetic resonance 
UNDERGRADUATE RESEARCH IN NATURAL AND CLINICAL SCIENCE AND TECHNOLOGY (URNCST) JOURNAL Read more URNCST Journal articles and submit your own today at: https://www.urncst.com

cholangiopancreatography (MRCP) was conducted, revealing biliary dilatation and CBD stone. An endoscopic retrograde cholangiopancreatography (ERCP) was later performed to remove the CBD stone.

Conclusion: In summary, patients with E.coli septicaemia may be more prone to bile duct stone formation especially in immunocompromised patients. A high index of suspicion should prompt early radiological investigations of the biliary system.

\section{A research study in immune-virology techniques and application of genotype identification in the diagnosis of chronic Hepatitis B infection}

Eimear Hennessy, MB, BCh, BAO Student, [1]

[1] Department of Molecular Virology Diagnostic \& Research Laboratory, Cork University Hospital, University College Cork, City, Ireland

Introduction: The Hepatitis B Virus (HBV) is an alarming global health problem today. An estimated 257 million people are chronic carriers worldwide. HBV is a partially double stranded DNA virus, which replicates via an RNA intermediate by reverse transcription, catalysed by the viral polymerase which lacks proof reading ability. This leads to sequence heterogeneity and the evolution of at least 9 genotypes and over $35 \mathrm{sub}$ genotypes. The aim of this study is to explore the genetic diversity of $\mathrm{HBV}$ and the laboratory techniques used in genotype identification of HBV.

Methods: 2 chronic HBV positive serum samples (HBsAg positive > 6 months, C.U.H). The HBV-DNA extracted was bi-directionally sequenced using primary and secondary PCR. Reverse Line Probe Hybridisation was used for genotype identification. Phylogenetic Analysis (MEGA 7 software) mapped the evolutionary history inferred, by using the Maximum Likelihood method based on the Tamura-Nei model. The Bootstrap Method calculated the statistical significance. A PubMed Nucleotide Pairway BLAST compared both genotypes to analyse the genetic similarities.

Results: The results of my research found both HBV Genotype A1 (accession number: AB010289) and HBV Genotype B2 (accession number: AB076678) positive serum samples to be present. The Pairway BLAST revealed 0\% gaps, 92\% identities and 2953bp between the aligned genotype A1 and B2. HBV genotype B2 is a recombinant genotype, the genetic framework is HBV genotype B with a precure region from genotype $\mathrm{C}$. Recombinant genotypes have a high mutation rate and correspond with more severe disease. Genotypes A1 and B2 have a relatively low mutation rate and better disease outcome in terms of response to antiviral therapy and rate of complications than other genotypes.

Conclusion: This research successfully achieved the application of genotype identification techniques to extract HBV DNA from the chronically infected serum samples. Our understanding of the molecular involvement and mutation variants of the HBV has been greatly deepened. Advancing our understanding of the HBV genotype distribution present in Ireland today is necessary to effectively prevent, treat and manage the hepatitis B infection.

\section{Detection of CTX-M 15 gene via LAMP reaction in Escherichia coli and its impact in antibiotic resistance in UTIs Ana Vaz, BSc Medical Sciences Student [1], Jeroen Nieuwland, PhD [1] [1] School of Applied Sciences, University of South Wales, Pontypridd, Cardiff, UK CF37 4BD, United Kingdom}

Introduction: Antibiotic resistance is a public health threat that is predicted to be a greater killer than cancer. This phenomenon has also been growing amongst uropathogens, the causative agents of UTIs, which are one of the most frequent bacterial infections [1]. Escherichia coli is the major uropathogen and its resistance to common antibiotics used to treat UTIs such as third-generation cephalosporins has become greater. Its resistance is associated with fact it carries ESBLs, predominantly the CTX-M 15 gene [2]. One of the main reasons for the spread of antimicrobial resistance is a poor antibiotic stewardship, due to lack of fast diagnostic test tools [1]. The viability of a point-of-care diagnostic tool based on LAMP was tested.

Methods: LAMP performs an ultra-rapid, specific and sensitive DNA synthesis, capable to detect infectious bacteria and their resistance profile. The feasibility of the detection of the CTX-M 15 gene of ESBL E. coli via LAMP was investigated by first, designing the LAMP primers and then setting up multiple LAMP reactions and gel electrophoresis to confirm the viability of the results. PCR was also utilised to confirm the efficiency of the primers before they were tested in LAMP.

Results: LAMP reaction revealed able to detect $E$. coli containing the CTX-M 15 gene resistant to certain $3 \mathrm{GCs}$ and to be more specific than PCR. The gene CTX-M 15 was identified in one of the colonies and its resistance to 3GCs confirmed by an antibiotic resistance test.

Conclusion: Many problems with primer-primer interactions, non-specific amplification and contamination occurred. The discovery of the ideal primer design, temperature reaction and false-positives results are the greatest disadvantages of LAMP. Sequencing results from the 3GCs resistant colonies proved bacteria's genetic plasticity by displaying variances in the DNA sequence of the same gene in distinct colonies. 
UNDERGRADUATE RESEARCH IN NATURAL AND CLINICAL SCIENCE AND TECHNOLOGY (URNCST) JOURNAL

Read more URNCST Journal articles and submit your own today at: https://www.urncst.com

\section{References}

[1] O'Neill J. Tackling drug-resistant infections globally: Final report and recommendations. HM Government; 2016.

[2] Peirano G, van Greune C, Pitout J. Characteristics of infections caused by extended-spectrum $\beta$-lactamase-producing Escherichia coli from community hospitals in South Africa. Diagn Microbiol Infect Dis. 2011 Apr; 69(4): 449-53. https://doi.org/10.1016/j.diagmicrobio.2010.11.011

\section{The contribution of a rapid multiplex PCR (rmPCR) blood culture identification panel to antimicrobial stewardship and patient outcomes}

Jack P. Finn Medical Student [1], Deirdre O'Brien MB, MRCSI, FRCPath [1, 2]

[1] School of Medicine, University College Cork, Ireland

[2] Department of Clinical Microbiology, Mercy University Hospital, Cork, Ireland

Introduction: With antibiotic resistance looming, efforts must be made to de-escalate and cease antibiotic therapy promptly while optimising outcomes for patients. Rapid diagnostic techniques may provide earlier evidence supporting safe de-escalation of antibiotic therapy than conventional blood culture techniques. This study aims to establish the impact of an rmPCR panel on the time from blood culture collection to communicating the result.

Methods: This retrospective cohort study consisted of two groups of patients with blood cultures that were positive for gram positive cocci; one from 2015 of 15 patients (before rmPCR) and one from 2017 of 19 patients (after rmPCR). The times of blood culture collection, arrival at lab and result communication were recorded and the intervals between each compared.

Results: In the 2015 cohort the mean time from collection of blood culture to result communication and time from culture arrival in lab to result communication were $36 \mathrm{hrs} 52$ minutes and 30 hours 20 minutes respectively. In the 2017 cohort the time from collection to report and arrival in lab to report were 22 hours 31 mins (39\% decrease) and 17 hours 52 minutes (41\% decrease) respectively. However, these reductions were not significant $(p=0.179$ and $p=0.13$, respectively) when the two groups were compared by Mann-Whitney $U$ tests. There was a significant time reduction $(p=0.033)$ found in the time from blood collection to delivery to lab between the two cohorts.

Conclusion: This study demonstrates the potential for rmPCR to reduce the time until a blood culture result is available to guide antibiotic de-escalation. It echoes trends shown in similar studies and while it does not achieve statistical significance, this is most likely due to small sample size.

36. Strategies for enhancing medical student resilience: A group concept mapping study Jessica L. Donohoe, [1] Colm O'Tuathaigh, [2] Margaret O'Rourke, [3] Sean Hammond, [4] Slavi Stoyanov [5]

[1] Medical Student, School of Medicine, University College Cork, Cork, Ireland, 903

[2] Senior Lecturer, Lead for Development of Undergraduate Research, University College Cork, Cork, Ireland, 903

[3] Senior Lecturer, Behavioural Science and Medicine, University College Cork, Ireland, 903

[4] Statutory Lecturer, Applied Psychological, University College Cork, Cork, Ireland, 903

[5] Faculty of Psychology and Educational Sciences, Open Universiteit in the Netherlands, Valkenburgerweg 177,6419 AT Heerlan, Netherlands

Introduction: Medical students are faced with constant stressors throughout their degree. Research has consistently shown that medical students have greater rates of mental-ill health in comparison to non-medical students. A need exists to explore the resilience strategies medical students employ to address these demands. Insight into these specific strategies may guide potential adaptation and incorporation of resilience-promoting interventions into the medical curriculum.

Methods: Group concept mapping was utilised incorporating qualitative and quantitative methodologies. A stratified sample of $3^{\text {rd }}$ year DEM and $2^{\text {nd }}$ year GEM medical students attending UCC were invited to take part in the GCM process on the group concept software. This process involved brainstorming $\left(1^{\text {st }}\right.$ step), categorisation $\left(2^{\text {nd }}\right.$ step) and rating $\left(3^{\text {rd }}\right.$ step) of resilience strategies the students employ to address stress during medical school. The data was analysed utilizing The Concept System ${ }^{\circledR}$ software through multidimensional scaling (MDS) and hierarchical clustering.

Results: 6 resilience strategy categories emerged organically from the data based on students coding of the text into perceived similarities. These categories included 'friends and family', 'de-stress through exercise/sport', 'extra-curricular non-medical activities', 'self-enabled distraction', 'organisation', 'caring for mental wellbeing'. The 'friends and family' category rated the highest amoung students in terms of effectiveness whereas the 'de-stress through sport and exercise' category rated the highest in value of importance to incorporate into a resilience based intervention programme.

Conclusion: Resilience strategies rated by medical students to be effective and important to incorporate in a resilience promoting, stress reduction programme are accessible and amenable for implementation into the medical curriculum. 
UNDERGRADUATE RESEARCH IN NATURAL AND CLINICAL SCIENCE AND TECHNOLOGY (URNCST) JOURNAL

Read more URNCST Journal articles and submit your own today at: https://www.urncst.com

37. Has there been a reduction in codeine-related intentional drug overdose presentations to Irish hospitals following national guidance in $\mathbf{2 0 1 0}$ ?

Emma Birchall, Medical Student [1], Ivan J. Perry, Professor Public Health [2], Eve Griffin, PhD [3]

[1] School of Medicine, University College Cork, Cork, Ireland

[2] Department of Epidemiology and Public Health, University College Cork, Ireland

[3] National Suicide Research Foundation, Cork, Ireland

Introduction: In Ireland, the most common method of hospital-presenting self-harm is intentional drug overdose (IDO). Concerns about the misuse of codeine products led to introduction of guidance for pharmacists in 2010, restricting supply of over-the-counter (OTC) codeine-containing products.

Our aim was to examine codeine-related IDO presentations to Emergency Departments before and after the introduction of guidance by the Pharmaceutical Society of Ireland in 2010.

Methods: IDO presentations to Emergency Departments, recorded by the National Self-Harm Registry Ireland between $1^{\text {st }}$ January 2007 and 31 st December 2013 were analysed. Event-based rates per 100,000 were calculated using national population data. Poisson regression models were used to assess rate changes between pre- and post-guidance periods.

Results: Between January 2007 and December 2013, a total of 57,759 IDOs were recorded. 4,789 (8.3\%) involved a codeine product. The rate of codeine-related IDOs was $21 \%$ lower in the period following implementation of the guidance (17.1 vs 13.5 per 100,000; IRR $=0.79 ; 95 \% \mathrm{CI}: 0.74-0.84)$. The rate of all IDOs decreased by $6 \%$ in the same period (0.94; 0.93-0.96). More pronounced reductions were seen in females than males (25\% vs $14 \%$ ) and among those aged under 25 compared to over 25 years $(29 \%$ vs $17 \%)$.

Conclusion: Our findings indicate that the guidance was associated with reductions in the rate of codeine-related IDOs. They provide evidence supporting restriction of potentially harmful medication as an effective strategy in suicide prevention. However, as overall rates of IDOs also fell during this period, the reductions may not be fully attributed to the guidance.

38. The association between monocyte subset numbers and significant disease on coronary angiography Power, R. [1], Cormican, S. [1, 2], Níc Aodha Bhuí, B. [3], Doran, J. [3], Short, P. [3], MacNeill, B. [3], Griffin, M.D. [1, 2] [1] Regenerative Medical Institute, National University of Ireland, Galway, Ireland

[2] Nephrology Department, Galway University Hospital, Newcastle Rd, Galway, H91 YR71, Ireland

[3] Cardiology Department, Galway University Hospital, Newcastle Rd, Galway, H91 YR71, Ireland

Introduction: Monocytes are keys cells in the development of atherosclerotic coronary artery disease (CAD) and are comprised of three distinct subsets [1]. Our group recently described HLA-DR ${ }^{\text {mid }}$ and HLA-DR ${ }^{\text {hi }}$ subpopulations within the intermediate subset [2]. We aimed to determine whether total monocyte or monocyte subset/subpopulation numbers are altered in individuals with CAD.

Methods: Ethics approval for this cross-sectional observational study was granted by the Clinical Research Ethics Committee at Galway University Hospital. Healthy volunteers $(\mathrm{HV}, \mathrm{n}=20)$ or individuals undergoing elective coronary angiography (ECA, $\mathrm{n}=42$ ) donated blood samples after informed consent. Peripheral blood mononuclear cells were isolated and monocyte subset numbers determined using multicolour flow cytometry. Clinical data, including angiography results, were recorded. Flow cytometry files were analysed using FlowJo ${ }^{\circledR}$ software and statistical analysis performed with GraphPad Prism ${ }^{\circledR}$ (unpaired t-test or Mann-Whitney-U test after normality testing).

Results: Participants undergoing ECA had higher total $(362,460$ cells $/ \mathrm{ml} v \mathrm{vs} .282,680 ; \mathrm{p}=0.0037)$, intermediate $(39,324 \mathrm{cells} / \mathrm{ml}$ vs.22,935; $\mathrm{p}=0.0001)$, nonclassical $(45,772$ cells $/ \mathrm{ml}$ vs.33,184; $\mathrm{p}=0.0357)$ and HLA-DR ${ }^{\text {hi }}$ intermediate $\left(25,136\right.$ cells $/ \mathrm{ml}^{2}$ vs. 14,$467 ; \mathrm{p}<0.0001)$ monocytes compared to HVs.

Individuals with CAD requiring further intervention had higher total $(419,520$ cells $/ \mathrm{ml} \mathrm{vs.346,260;} \mathrm{p}=0.0060)$, classical $(344,078$ cells $/ \mathrm{ml}$ vs. 259,$951 ; \mathrm{p}=0.0178)$ and HLA-DR ${ }^{\text {hi }}$ intermediate $(31,284$ cells $/ \mathrm{ml} v \mathrm{vs} .20,164 ; \mathrm{p}=0.0326)$ monocytes than those not requiring intervention after diagnostic angiography (Table 1).

Shaw et al. | URNCST Journal (2020): Volume 4, Issue 3

Page A19 of A46

DOI Link: https://doi.org/10.26685/urnest.182 
UNDERGRADUATE RESEARCH IN NATURAL AND CLINICAL SCIENCE AND TECHNOLOGY (URNCST) JOURNAL

Read more URNCST Journal articles and submit your own today at: https://www.urncst.com

Table 1: Total monocyte subset/ subpopulation numbers by individuals requiring intervention vs. those who didn't require further intervention post ECA. *Asterisk indicates statistical significance.

\begin{tabular}{|c|c|c|c|}
\hline & Intervention $(\mathbf{n}=\mathbf{1 2})$ & No Intervention $(\mathbf{n}=\mathbf{3 0})$ & p-value \\
\hline Total Monocytes & 419520 & 346260 & $.0060^{*}$ \\
\hline Classicals & 344078 & 259951 & $0.0178^{*}$ \\
\hline Intermediates & 42854 & 39324 & $.4909^{*}$ \\
\hline Nonclassicals & 54773 & 39025 & .0567 \\
\hline HLA-DR $^{\text {id }}$ & 9737 & 14867 & .2427 \\
\hline HLA-DR $^{\text {hi }}$ & 31284 & 20164 & $.0326^{*}$ \\
\hline
\end{tabular}

Conclusion: We observed that individuals with CAD requiring intervention have increased total, classical, and HLA-DR ${ }^{\text {hi }}$ intermediate monocyte numbers compared to those who underwent angiography but didn't require further intervention. Consideration of total monocyte or monocyte subset/subpopulation numbers could be a useful adjunct to current risk stratification systems.

\section{References}

[1] Zawada AM, Rogacev KS, Schirmer SH, Sester M, Bohm M, Filser D, Heine GH. Monocyte heterogeneity in human cardiovascular disease. Immunobiology 2012; 217(12): 1273-84. https://doi.org/10.1016/j.imbio.2012.07.001

[2] Connaughton Eanna P, Naicker S, Hanley Shirley A, Slevin S, Eykelenboom JK, Lowndes NF, O’Brien T, Ceredig R, Griffin MD, Dennedy MC. Phenotypic and functional heterogeneity of human intermediate monocytes based on HLA-DR expression. Immunol Cell Biol. 2018 Mar 5 [Online ahead of print]. https://doi.org/10.1111/imcb.12032

39. Lung tumor diagnoses in people living with HIV in a large clinical centre in Canada: A retrospective review of cases over 3 decades

Beatrice Bichara, BSc Student [1], Jean-Pierre Routy, PhD [2, 3], Nicole Ezer, PhD [4, 5], Cecilia T. Costiniuk, PhD [2, 6]

[1] Department of Medicine, University of Ireland, Galway, Galway Co., Ireland

[2] Chronic Viral Illness Service and Research Institute of McGill University Health Centre, Montreal, Quebec, Canada H4A $3 \mathrm{JI}$

[3] Division of Hematology, McGill University Health Centre, Royal Victoria Hospital: Glen Site, Montreal, Quebec, Canada H4A $3 \mathrm{JI}$

[4] Division of Respirology, McGill University Health Centre, Royal Victoria Hospital: Glen Site, Montreal, Quebec, Canada H4A $3 J 1$

[5] Clinical Outcomes Research and Evaluation (CORE), Research Institute of the McGill University Health Centre, Montreal Quebec, Canada

[6] Division of Infectious Diseases, McGill University Health Centre, Royal Victoria Hospital: Glen Site, Montreal, Quebec, Canada H4A 3J1

Introduction: Lung cancer is a leading cause of non-AIDS defining cancers and the most frequent cancer- related death in people living with HIV (PLWH). Our aim was to conduct a review of cases of lung cancer in one academic center to better understand areas needed for quality improvement at the McGill University Health Centre (MUHC).

Methods: The clinical database the of the Chronic Viral Illness Service (CVIS) was queried to identify individuals who had a pathology-confirmed diagnosis of lung cancer from 1980-2018.

Results: 21 PLWH received a pathology-confirmed diagnosis of primary lung cancer. Median age was 55 years and $19 \%$ were female. 3, 13, and 5 cases were diagnosed between 1990-1999, 2000-2010 and 2011-2018 respectively. Median CD ${ }^{4}$ count was 197 cells/mm3 [70, 450] and 52\% were on ARVs with suppressed viral loads. Type of lung cancer included: non-small cell lung cancer (15 cases), Small-cell Lung cancer accounted (4 cases) and bronchial carcinomas ( 2 cases). Metastases at diagnosis were present in $11(52 \%)$ persons, 2 underwent surgical lobectomy and 7 received radiation therapy alone or combined with chemotherapy. Mean overall survival was 8 months (IQR 1.75, 11.75) and 4 persons survived without recurrence. 
UNDERGRADUATE RESEARCH IN NATURAL AND CLINICAL SCIENCE AND TECHNOLOGY (URNCST) JOURNAL Read more URNCST Journal articles and submit your own today at: https://www.urncst.com

Conclusion: A high proportion (52\%) of lung cancers were diagnosed at advanced clinical stages. The implementation of a lung cancer screening program, a tobacco cessation program and the integration of cancer care in the HIV setting at the MUHC should set the stage for earlier diagnosis and treatment of lung cancer, thus reducing morbidity and mortality in PLWH.

40. Prevalence of risk of obstructive sleep apnoea among patients with cardiac diseases in an Irish population ZY Tai, MB, BCh, BAO Student [1], DM Kerins, MD, FACC, FAHA, FESC, FASE, FBPHS [2, 3]

[1] College of Medicine and Health, University College Cork, Cork, Co Cork, Ireland, T12 YT2O

[2] Department of Pharmacology and Therapeutics, University College Cork, Cork, Co Cork, Ireland, T12 YT20

[3] Department of Cardiology, Mercy University Hospital, Cork, Co Cork, Ireland, T12 WE28

Introduction: Obstructive Sleep Apnoea (OSA) is a commonly undiagnosed condition. Risk factors include hypertension and diabetes mellitus, which are shared with many cardiovascular diseases (CVD). OSA has many cardiovascular complications. However, the prevalence of OSA in patients with cardiovascular disease is poorly documented. Thus, the aim of this project is to study the prevalence of patients with CVD with high risk for OSA, and to stratify into specific cardiac diseases, namely ischaemic heart disease, arrhythmia, valvular heart disease and congestive heart failure.

Methods: This study was conducted in the Cardiovascular Outpatient Clinic in Mercy University Hospital between September 2018 and March 2019. The Berlin Questionnaire was administered by a trained medical student to patients with cardiovascular diseases. The patients are then stratified into either high risk or low risk for OSA.

Results: In a study population of 150 patients, 118 were male and 32 were female. The mean age was $66.5 \pm 12.7$ years, and mean BMI was $27.0 \pm 4.31 \mathrm{~kg} / \mathrm{m}^{2} .32 \%$ of the study population are classified as high risk for OSA. Stratifying by type of heart disease, the percentage of high risk OSA is $42.4 \%$ for ischemic heart disease, $17.1 \%$ for arrhythmia, $20 \%$ for valvular heart disease, and $66.7 \%$ for congestive heart failure.

Conclusion: There is a significant proportion of patients with cardiovascular diseases who are at high risk of OSA, and this would merit clinicians' attention and assessment. More research is warranted to study the incidence, morbidity and mortality of OSA in this patient group.

\section{Acceptability and effectiveness of a mindfulness App and Heart-Rate monitor for U.S. Veterans Lorcan O'Byrne, BSc, MB, BCh, BAO Student [1], Rumi K. Price, PhD [2] \\ [1] UCD School of Medicine, University College Dublin, Belfield, Dublin 4, Ireland \\ [2] School of Psychiatry, Washington University of St Louis School of Medicine, Missouri, United States}

Introduction: Several barriers (stigma, financial concerns, geographic constraints and medication concerns) discourage U.S. Veterans from seeking effective posttraumatic stress disorder (PTSD) treatment. Thus, despite the availability of evidence-based treatments, rates of PTSD remain high in this population, highlighting a need for innovative management tools. Previous studies have shown mobile applications to be efficacious in treating PTSD symptoms. Mindset is a similar application, designed to help users manage their mental health symptoms; however, it is novel as it utilizes heart rate-related data via a smartwatch to monitor user's stress level and deploy app e-therapies.

Methods: This pilot study evaluates the effectiveness and acceptability of Mindset and the applications capacity in managing PTSD symptoms. Twenty-four community-residing Veterans meeting inclusion criteria completed both baseline and follow-up interviews. Following baseline interview, participants used the Mindset app and related heart rate watch continuously for approximately one month until their follow-up interview. Interview assessments included pre- and post-deployment experiences, standardized screeners for PTSD (PCL-M), anxiety (GAD-7), depression (PHQ-9), alcohol use problems (AUDIT-10), and user experience with Mindset, among others.

Results: Using SAS 9.4, a significant decrease ( $\mathrm{p}<0.05$ ) was found in PCL-M, PHQ-9 and AUDIT-10 between baseline and follow-up interviews. Respondents reported moderate to high acceptance and satisfaction with Mindset features. Mindset use may be associated with decreasing symptoms of PTSD and alcohol abuse in this sample of U.S. Veterans

Conclusion: This study highlights a Mindfulness app, such as Mindset, as a useful tool to augment existing therapies for patients suffering from post-traumatic stress disorder. 
UNDERGRADUATE RESEARCH IN NATURAL AND CLINICAL SCIENCE AND TECHNOLOGY (URNCST) JOURNAL Read more URNCST Journal articles and submit your own today at: https://www.urncst.com

42. The relationship of strength and power measures at 9 months post anterior cruciate ligament reconstruction surgery and patient reported outcomes at 2 years: A research study David P. Synnott, BSc Student [1], Katherine A.J. Daniels PhD [2, 3], Enda King [2, 4], Éanna Falvey PhD [1, 2]

[1] Department of Medicine, University College Cork, Cork, Ireland T12 AK54

[2] Sports Medicine Department, Sports Surgery Clinic, Santry, Dublin 9, Ireland D09 C523

[3] Queen's School of Engineering, University of Bristol, Bristol, United Kingdom BS8 1TR

[4] Department of Life Sciences, University of Roehamptom, London, United Kingdom SW15 4JD

Introduction: One in five professional athletes do not return to their pre-injury level of participation following ACLR Surgery [1]. 23\% of those who do, suffer re-injury [2]. A lack of consensus around return-to-sport guidelines may contribute to re-injury rates and fear-avoidance behaviour. This study aims to define the relationship between strength and power measures at 9 months post-op and determine if these measures can be used to direct patients on when it is safe to return to sport.

Methods: A prospective cohort study was performed of 240 consecutive, eligible ACLR patients at the Sports Surgery Clinic, Dublin. A single-leg drop jump (SLDJ), a single-leg counter-movement jump (SLCMJ), and isokinetic testing were carried out in a 3D motion capture and muscle dynamometry laboratory 9 months post-surgery. At 2 years, PROMs were evaluated by 5 questionnaires, 4 validated. Multiple logistic regressions were used in analysing data and the Benjamini-Hochberg procedure employed due to multiple testing.

Results: Twenty four percent of participants were at participation levels worse than pre-injury. SLDJ and SLCMJ heights on the injured leg had Marx Score (validated questionnaire) regression coefficients of $0.275(\mathrm{p}=0.000)$ and $0.243(\mathrm{p}=0.001)$. A $1 \mathrm{~cm}$ increase in injured SLDJ height was associated with a $10 \%$ decrease in the odds $(\mathrm{OR}=0.90,95 \% \mathrm{CI} 0.83$ to 0.99$)$ of a worse level of performance. Isokinetic results showed no correlation with PROMs.

Conclusion: Superior jump scores on the injured leg at 9-months post-ACLR are associated with improved PROMs at 2 years. Isokinetic strength results do not have an association with PROMs.

\section{References}

[1] Lai CCH, Ardern CL, Feller JA, Webster KE. Eighty-three percent of elite athletes return to preinjury sport after anterior cruciate ligament reconstruction: a systematic review with meta-analysis of return to sport rates, graft rupture rates and performance outcomes. Br J Sports Med. 2018 Jan; 52(2): 128-38. https://doi.org/10.1136/bjsports-2016-096836

[2] Wiggins AJ, Grandhi RK, Schneider DK, Stanfield D, Webster KE, Myer GD. Risk of Secondary Injury in Younger Athletes After Anterior Cruciate Ligament Reconstruction: A Systematic Review and Meta-analysis. Am J Sports Med. 2016 Jul; 44(7): 1861-76. https://doi.org/10.1177/0363546515621554

43. 22Q11 deletion syndrome (22q11DS)- Review of the mental health profiles of children referred to a specialist clinic for a psychiatric assessment

Maryam Albreiki, [1], Veselina Gadancheva [1, 2], Fiona McNicholas [1, 2]

[1] UCD School of Medicine, University College Dublin, Belfield, Dublin 4, Ireland

[2] CHI Psychiatry Department, Children's Health Ireland at Crumlin, Dublin 12, Ireland

Introduction: Di George syndrome or 22Q11DS is the most common micro-deletion syndrome in humans. Children with 22Q11DS may present with a range of congenital abnormalities and developmental delay [1]. Psychiatric conditions such as autistic spectrum disorder (ASD), Attention Deficit Hyperactivity Disorder (ADHD), anxiety and psychosis are prevalent in this group [2]. The Child Behavioral Checklist (CBCL), completed by a caregiver, and the Teacher's Report Form (TRF) are commonly used to evaluate children's and adolescents' emotional and behavioral difficulties [1]. Based on CBCL and TRF scores, children could be screened for a spectrum of mental health difficulties.

Aim \& Method: This study aims to establish mental health profiles of children with 22q11DS, referred for a psychiatric assessment, based on CBCL and TRF questionnaires. Anonymous data were extracted and analysed with SPSS.

Results: A total of 35 children (20 males; 15 females) aged 4-16 (mean age 9.11, SD=3.26) were seen in the clinic. Based on the CBCL score for Internalising Problems N=20, 69\% of the children were within the borderline/clinical range. $\mathrm{N}=13$, 45\% scored in the borderline/clinical range for Externalising Problems.

TRFs were available for 23 children. $\mathrm{N}=13,59 \%$ of children had borderline/clinical scores on internalising problems. The externalising score was in the borderline/clinical range for $\mathrm{N}=3,13 \%$ of the young people. Following an assessment $\mathrm{N}=21,60 \%$ out of 35 children were given a clinical diagnosis predominantly anxiety disorder and ADHD.

Conclusion: Interestingly, more Externalising Problems were reported by parents than teachers. CBCLs and TRF are useful tools contributing to a detailed clinical assessment.

Shaw et al. | URNCST Journal (2020): Volume 4, Issue 3

DOI Link: https://doi.org/10.26685/urnest.182 
UNDERGRADUATE RESEARCH IN NATURAL AND CLINICAL SCIENCE AND TECHNOLOGY (URNCST) JOURNAL

Read more URNCST Journal articles and submit your own today at: https://www.urncst.com

\title{
References
}

[1] Klaassen P, Duijff S, Sinnema G, Beemer F, Swanenburg de Veye H, Vorstman J. Behavioral phenotype in children with 22q11DS: Agreement between parents and teachers. Psychological Assessment. 2015; 27(1):272-279. https://doi.org/10.1037/a0038102

[2] Briegel W, Schneider M, Schwab K. 22q11.2 deletion syndrome: behaviour problems of children and adolescents and parental stress. Child Care Health Dev. 2008 Nov; 34 (6): 795-800. https://doi.org/10.1111/j.1365-2214.2008.00850.x

\author{
44. Sequential cochlear implantation in children: Functional outcomes and the effect of inter-implant delay and \\ device parameters \\ C. McCormack, Student [1], C. Simoes-Franklin [2], R. O’Halpin [2], E. Walshe [2], \\ F. Glynn [2], P. Walshe [2], L. Viani [1, 2] \\ [1] Royal College of Surgeons in Ireland, Dublin 2, Ireland \\ [2] National Hearing Implant and Research Centre, Beaumont Hospital, Dublin 9, Ireland
}

Introduction: Cochlear implants are used to enable children with severe to profound sensorineural hearing loss to hear. In 2014, funding was approved for paediatric bilateral cochlear implants in Ireland. Therefore, children who had received one implant (CI1) were offered a second (bilateral sequential implantation). The purpose of this study is to evaluate the effect of inter-implant delays (IID) on the success of a sequential implant to determine if children should always receive a second cochlear implant.

Methods: The focus of this longitudinal study is a population of 207 children who underwent bilateral sequential surgery for a second cochlear implant (CI2). Patients were divided into three groups on the basis of their IID, ranging from 0.1 to 15.7 years. Outcomes were assessed using BKB sentence testing of speech perception and specific device parameters: device usage, dynamic range and generation of implant. Data analysis was completed using standard student t-tests and $\mathrm{p}$-values of $<0.05$.

Results: IIDs < 10 years (Group 1 and Group 2) showed significant improvements $(\mathrm{p}<0.01)$ in speech perception bilaterally when compared with unilateral performance of the first implant and were more inclined to use their second implant. However, longer IIDs >10years (Group 3) were associated with no improvement in speech perception, decreased device usage and narrower dynamic ranges.

Conclusion: Inter-implant delay is known to be an important predictor of outcomes in sequential cochlear implant recipients. Patients should be made aware of the complexities of receiving a second implant so that they can make an informed choice about sequential implantation.

45. Clinical profile of adult patients with type 1 diabetes attending an Irish hospital S Gannon [1], D O’Keeffe [1, 2], ES O'Sullivan [1, 2]

[1] School of Medicine, National University of Ireland, Galway, Ireland

[2] Centre for Endocrinology, Diabetes and Metabolism (CDEM), Galway University Hospital, Galway, Ireland

Introduction: Type 1 diabetes is an autoimmune disorder associated with increased mortality and lower quality of life. The aim of this study was to create a profile of the clinical values of all patients with type 1 diabetes, who attend an Irish Hospital. This will then be compared to the ADA recommendations [1], other similar studies as well as a profile of the same location done at an earlier date [2]. The clinical values were compared between types of insulin regime (Multiple Daily Injections, premixed, Continuous Subcutaneous Insulin Infusion).

Methods: This was a retrospective cross-sectional analysis of patients with type 1 diabetes, who attended the centre for endocrinology, diabetes and metabolism between June 2013 and June 2019.

Results: There were 1501 patients managed at the centre within the timeframe, $49.23 \%$ male, 50.77\% female. 1311 (87.34\%) patients managed their insulin with Multiple Daily Injections, $10 \%$ with CSII and $2.66 \%$ with premixed. The mean HbA1c was $68.04 \mathrm{mmol} / \mathrm{mol}$, with $15.35 \%$ of the cohort $<53 \mathrm{mmol} / \mathrm{mol} .92 .88 \%$ had a dBP $<90 \mathrm{mmHg}, 78.68 \% \mathrm{has}$ a sBP $<140 \mathrm{mmHg}$. The total cholesterol, HDL-cholesterol, LDL-cholesterol and triglycerides were within ADA recommendations for 75.12\%, $88.02 \%, 61.89 \%$ and $81.42 \%$ of the population respectively. The mean HbA1c for CSII users was $62.95 \mathrm{mmol} / \mathrm{mol} \mathrm{compared}$ to $69.26 \mathrm{mmol} / \mathrm{mol}$ for non-CSII users, T-test returning a p-value of <.0001.

Conclusion: Most of the targets had a majority of patients meet them except HbA1c and BMI. This is comparable to other studies. The lower mean HbA1c for patients using CSII supports its efficacy in improving glycaemic control.

Shaw et al. | URNCST Journal (2020): Volume 4, Issue 3

DOI Link: https://doi.org/10.26685/urnest.182 
UNDERGRADUATE RESEARCH IN NATURAL AND CLINICAL SCIENCE AND TECHNOLOGY (URNCST) JOURNAL

Read more URNCST Journal articles and submit your own today at: https://www.urncst.com

\section{References}

[1] American Diabetes Association. Standards of Medical Care-2019. Diabetes Care. 2019; 42(supplement 1): S61-S70. https://doi.org/10.2337/dc19-S006

[2] Cotter TG, Dinneen SF, Healy DA, Bell MJ, Cunningham A, O'Shea PM, Dunne F, O'Brien T, Finucane FM. Glycaemic control is harder to achieve than blood pressure or lipid control in Irish adults with type 1 diabetes. Diabetes Res Clin Pract., 2014 Dec; 106 (3): e56-9. https://doi.org/10.1016/j.diabres.2014.09.036.

46. Prevalence and significance of anginal equivalent in patients attending a Rapid Access Chest Pain Clinic Bruno M. Chan Chin, MB, BCh, BAO Student [1], Ronan J. Curtin, MB, BCh, NUI [2]

[1] Department of Medicine, University College Cork, Cork, Ireland

[2] Department of Cardiology, Cork University Hospital, Cork, Ireland

Introduction: Coronary artery disease (CAD) is the main cause of death globally. While patients with CAD often present with typical angina, there is a subset of patients that present with dyspnoea instead, termed "anginal equivalent" [1]. The aim of this project is to establish the prevalence of patients referred to the RACPC with an anginal equivalent and to determine its significance.

Methods: A retrospective analysis of summary letters from the RACPC identified 397 individuals between January and April 2017. These were divided into three groups according to their presenting complaint, typical angina (TypAng), dyspnoea or reference group. The demographic characteristics, co-morbidities and outcome of their exercise stress test were recorded and the results from coronary angiograms performed in patients with abnormal stress tests were analysed to determine the proportion of patients with CAD.

Results: From the 397 patients identified, $11.6 \%$ of individuals presented with dyspnoea, $6.8 \%$ with TypAng and the majority of patients $(81.6 \%)$ were in the reference group. There was no statistically significant difference between TypAng and dyspnoea in mean age, hypertension, diabetes or dyslipidaemia. However, TypAng had a higher number of abnormal stress tests than dyspnoea $(29.6 \%$ vs $10.9 \%, \mathrm{p}=0.043)$. Results of coronary angiograms showed that $18.5 \%$ of TypAng had obstructive CAD, compared to $2.2 \%$ of dyspnoea $(\mathrm{p}=0.015)$.

Conclusion: The prevalence of dyspnoea in the RACPC between January and April 2017 was higher than TypAng. However, TypAng was associated with a higher rate of obstructive CAD than dyspnoea, demonstrating a lower yield for cardiac testing in the latter population.

\section{Reference}

[1] Nakanishi R, Rana JS, Rozanski A, Cheng VY, Gransar H, Thomson LE, et al. Relationship of dyspnea vs. typical angina to coronary artery disease severity, burden, composition and location on coronary CT angiography. Atherosclerosis. 2013; 230(1): 61-6. https://doi.org/10.1016/j.atherosclerosis.2013.06.008

\section{Head and neck cancer in IV drug users: A retrospective study}

Matthew Condon, BSc Student [1], Fergal G. Kavanagh, MD [2], Paul Lennon, MD [1, 2].

[1] School of Medicine, Trinity College Dublin, College Green, Dublin 2, Ireland

[2] Department of Otorhinolaryngology, Head and Neck Surgery, St James Hospital, James Street, Dublin 8, Ireland

Introduction: While smoking and alcohol are well recognized as risk factors for developing head and neck cancer (HNC), the literature is sparse on the impact IV drug use has on patients with the disease. Our aim was to examine the presentation by age, site, risk factors and stage, and to analyse the outcomes of both current and ex-IVDU.

Methods: A retrospective observational review of known current and former IVDU, as well as those enrolled in methadone programmes, identified in our institution over a 10 year period was undertaken. We compared the presentation and outcomes to an audit of all HNC patients treated in 2016 at our institution.

Results: Thirty-one patients were identified. The average age of these patients was 46 years vs 62.5 years in the 2016 audit group $(\mathrm{p}<0.001)$. The most common malignancy was laryngeal cancer followed by oral cancer. Supraglottic tumours, and tumours of the floor of the mouth were disproportionately common in the IVDU group. The distribution of staging was similar across the two groups. Cigarette smoking (24 cases) and past/current alcohol abuse (18 cases) were common. Patients were often co-morbid with HCV (14 cases) and HIV (5 cases) infections.

Conclusion: Our results suggest an earlier presentation, with distinct sites of distribution of HNC in the IVDU population, when compared to the general population. Further research is required to examine IV drug use as an independent risk factor for the development of HNC.

Shaw et al. | URNCST Journal (2020): Volume 4, Issue 3

DOI Link: https://doi.org/10.26685/urnest.182 
UNDERGRADUATE RESEARCH IN NATURAL AND CLINICAL SCIENCE AND TECHNOLOGY (URNCST) JOURNAL Read more URNCST Journal articles and submit your own today at: https://www.urncst.com

\section{Coeliac and thyroid screening in children with Type 1 Diabetes}

Shaira Wignarajah, MD Student [1], Victoria Griffiths, MD Student [1], Niall Dalton, MD Student [1], Julie Evers, MD Student [1], Susan Giblin, PHD [1], Aisha Iajaz [2], Orla Neylon, MD [1, 2], Paul Scully, MD [1], Clodagh O'Gorman, MD [1, 2]

[1] Department of Paediatrics, Graduate Entry Medical School, University of Limerick, Limerick, Ireland, V94 T9PX

[2] Department of Paediatrics Endocrinology, University Hospital Limerick, Limerick, Ireland, V94 F858

Introduction: Coeliac disease and thyroid disorders are significantly higher in patients with Type 1 Diabetes (T1D). Thus, appropriate screening of children with T1D patients is crucial in optimising diabetes management. This study aimed to measure adherence to current screening practice for coeliac disease and thyroid disorders in children with T1D.

Methods: This study was a retrospective analysis conducted within the regional T1D paediatric outpatient clinic at University Hospital Limerick and analysed children with T1D who attended the clinic between June and August 2019. A chart review for each attendee was undertaken and examined for demographics, diabetes diagnosis and previous coeliac and thyroid screening data. Results: 76 eligible children attended the outpatient clinic during the study period. Demographic data showed a mean age of 12.1 years (range 3-18 years), 45\% male gender, and a mean duration of diabetes of 7.3 years. Further analysis showed that $98.7 \%$ underwent thyroid screening for thyroid stimulating hormone (TSH), with $82 \%$ undergoing thyroid peroxidase antibody testing. $96 \%$ of attendees were screened for coeliac disease by tissue transglutaminase (TTG), with $9 \%$ showing an elevated TTG level. $75 \%$ of attendees were concurrently tested for IgA levels.

Conclusion: There is close to universal screening for thyroid and coeliac disease within the cohort examined. Concurrent IgA level measurements was undertaken in only three out of four attendees, but the remainder may have had a previously documented normal IgA level. Auditing and adherence to screening guidelines optimises the screening practice for thyroid and coeliac dysfunction in a paediatric T1D population.

49. Democratising science: Development of low-cost, flexible and open-source lab tools Niamh Burke, BSc [1], George O.T. Merces BSc [1, 2], Amy Courtney BSc [1, 2], Mark Pickering B.A, PhD [1, 2]

[1] UCD School of Medicine, University College Dublin, Dublin, Leinster, Dublin 4, Ireland

[2] UCD Centre for Biomedical Engineering, University College Dublin, Leinster, Dublin 4, Ireland

Introduction: Many modern research and analytical tools are characterised by high purchase and running costs, putting them out of reach of many, particularly in developing countries. However, the advent of recent technologies, such as 3D printing and inexpensive microcontrollers (Arduino) and single-board computers (Raspberry Pi, RPi) allows the deployment of low-cost, simple yet powerful tools.

Methods: To test the principal of low-cost manufacture, we developed three prototypes of commonly used analytical equipment. The first device was a portable centrifuge based on a brushless DC motor with 3D printed rotor in an extruded aluminium frame. The second was a water quality monitor capable of long-term logging of $\mathrm{pH}$, temperature, and volume with remote wireless monitoring. The third was a dual view automated microscope, based on the 3D printed open flexure design which allowed simultaneous low and high magnification viewing of the same sample, utilising 3D gesture control to minimize vibration.

Results: All three devices were manufactured at significantly lower cost than commercial devices ( €30: centrifuge, $€ 150$ : microscope, $\sim € 50$ : water monitor), and all devices were shown to be functional with performance comparable to commercial devices.

Conclusion: We present a group of lab tools that are affordable and adaptable, and easily made without specialised skills. All three systems can be easily customised to suit the users' needs, including battery operation for diagnostic and research use in the field. This approach may place laboratory tools in the hands of many users for the first time, opening science to a new and diverse population. 
UNDERGRADUATE RESEARCH IN NATURAL AND CLINICAL SCIENCE AND TECHNOLOGY (URNCST) JOURNAL Read more URNCST Journal articles and submit your own today at: https://www.urncst.com

50. Can the Wender Utah Rating Scale (WURS)-61 predict other mental health disorders in adulthood except ADHD? Miss Cliodhna Hanley [1, 2], Dr Faisal Saleem [1], Dr Ignazio Graffeo [1], Prof Geraldine McCarthy [1], Prof Fiona McNicholas [2], Dr Dimitrios Adamis [1]

[1] Sligo Mental Health Services, Clarion Rd, Sligo, Ireland

[2] University College Dublin, Dublin, Ireland

Introduction: The WURS is a widely used retrospective questionnaire for detection of childhood ADHD symptoms in adults presenting for ADHD evaluations. Our aim was to find out if certain symptoms during childhood (including ADHD) are associated with specific mental disorders in adulthood.

Methods: Consecutive patients attending outpatient clinics in Sligo/Leitrim area, and people without mental disorders (assessed with GHQ-12) had completed the WURS-61. ADHD was diagnosed using Conners' scale and psychiatric evaluation. Other psychiatric disorders have been classified according to ICD-10 using clinical criteria, and grouped into major categories. Results: Total sample 726 (630 with mental disorders and 96 without). Mean age 39.8, SD: 12.9, 54\% females. There were not significant differences between cases and controls in terms of age and gender. Parallel analysis indicated that the best factor solution of the sample was the one with five factors. Using the factors scores and after cross-tabulation we found that: items of WURS which indicate conduct behaviours during childhood are significantly associated with disorders due to psychoactive substance use, items which indicate conduct symptoms and items which indicate impulsivity, emotional lability and distress are associated with personality disorders, items which indicate impulsivity, emotional lability and distress plus disorganisation are significantly associated with ADHD, and items which indicate popularity/sociability were significantly associated with the "normal" controls.

Conclusion: There is overlap of symptoms between ADHD, personality disorders, and substance abuse during the adulthood. Personality disorders and psychoactive substance use disorders perhaps can be detected at earlier stages during the childhood.

\section{Low and high LET radiation induced changes in tumour cells invasion under hypoxia Shannen Smyth, BSc, Medical Student [1], Pankaj Chaudhary, PhD [2] and Kevin M Prise [2] \\ [1] Medical Student, Queen's University, Belfast, Northern Ireland, BT7 1NN \\ [2] Centre for Cancer research and Cell Biology, Queen's University Belfast, Northern Ireland, BT71NN}

Introduction: Hypoxia is a common characteristic of solid tumours; proven to be associated with increased radioresistance, cell invasion and activation of the epithelial to mesenchymal transition (EMT) [1]. Low linear energy transfer (LET) radiation has shown to be insufficient at overcoming these hypoxia-associated barriers [2]. High LET radiation has displayed the ability to overcome hypoxia-mediated radioresistance, however its effects on cell invasion remain unclear.

Methods: We used normal prostate epithelium (PNT2C2) and metastatic prostate cancer cells (DU145) to study effects of low and high LET radiations under hypoxia. $225 \mathrm{kVp}$ X-rays were used for low LET radiations. High LET radiations were performed using Americium 241 Alpha particle source, with $5 \mathrm{MeV}$ of energy. We detected HIF-1 $\alpha$ protein expression using western blots. Radiation induced cell death was studied using the clonogenic assay. Cell migration and invasion was assessed using wound scratch assay. EMT was studied using immunofluorescent detection of E-cadherin and $\mathrm{N}$ cadherin.

Results: Both, the normal (PNT2C2) and metastatic prostate epithelial cells (DU145) showed upregulation of HIF-1 $\alpha$ under hypoxic conditions. Low LET radiations under hypoxia in both cell lines showed an increased cell survival, invasion and EMT. High LET irradiation led to an enhanced cell death and reduced invasion however, we noticed slight increase in the transition of epithelial to mesenchymal cells under oxic and hypoxic conditions.

Conclusion: Cells irradiated with low LET radiation under hypoxia displayed radioresistance, increased cell invasion and EMT. High LET radiation induced more cell-killing and less invasion than low LET radiations however, effects on the induction of the EMT require further investigation.

\section{References}

[1] Kim Y, Lin Q, Glazer PM, Yun Z. Hypoxic Tumor Microenvironment and Cancer Cell Differentiation. Curr Mol Med. 2009 May; 9(4): 425-434. https://doi.org/10.2174/156652409788167113

[2] Shannon AM, Bouchier-Hayes DJ, Condron CM, Toomey D. Tumour hypoxia, chemotherapeutic resistance and hypoxiarelated therapies. Cancer Treatment Reviews. 2003 Aug; 29(4): 297-307. https://doi.org/10.1016/S0305-7372(03)00003-3 
UNDERGRADUATE RESEARCH IN NATURAL AND CLINICAL SCIENCE AND TECHNOLOGY (URNCST) JOURNAL

Read more URNCST Journal articles and submit your own today at: https://www.urncst.com

52. Incidence and aetiology of stroke in patients with non-valvular atrial fibrillation on anticoagulation: A research study

Helena hobbs, BM BS student [1], Paul Cotter, MB, BAO, BCh, BMed Sci (NUI), MPhil, MD (Cantab), FRCPI [2]

[1] Graduate Entry Medical School, University of Limerick, Limerick, Ireland

[2] Department of General Medicine, St. Luke's Hospital, Kilkenny, Ireland

Introduction: Non-Valvular Atrial Fibrillation (NVAF) is a major risk factor for stroke, sudden death and heart failure [1]. An ischaemic stroke secondary to AF carries high mortality and morbidity. There is strong evidence recommending the use of Nonvitamin $\mathrm{K}$ antagonist Oral Anticoagulant (NOAC) over warfarin for NVAF for anticoagulation. The objective was to investigate the aetiologies of stroke in patients with $\mathrm{AF}$ on anticoagulation.

Methods: This study retrospectively identified adult (aged 18-99) patients who presented with a stroke (ischaemic and haemorrhagic) to a tertiary referral centre who had known AF and were on anticoagulation (warfarin, NOAC e.g. apixaban, dabigatran, rivaroxaban or edoxaban) between 2013 and 2017 inclusive. The National Stroke Registry was used to identify these patients.

Results: 67 patients were admitted with a stroke that had known AF and were on anticoagulation. 82\% ( $\mathrm{n}=55)$ of these patients admitted suffered an ischaemic stroke, $18 \%(\mathrm{n}=12)$ suffered a haemorrhagic stroke. Of these patients; 52 were anticoagulated with warfarin and 15 with a NOAC. The findings for ischaemic stroke; $60 \%$ were due to sub-therapeutic INR, $13 \%$ undetermined, $11 \%$, stopped for medical reason, $9 \%$ non-compliant, $5 \%$ held for procedure and $2 \%$ sub-therapeutic dose.

Conclusion: The leading cause of ischaemic stroke in NVAF patients was sub-therapeutic INR on warfarin.

\section{Reference}

[1] Kirchhof, P, Benussi, S, Kotecha, D, Ahlsson, A, Atar, D, Casadei, B, et al. 2016. ESC Guidelines for the management of atrial fibrillation developed in collaboration with EACTS. Eur J Cardiothorac Surg. 2016 Nov; 50(5): e1-e88. http://doi.org/10.1093/ejcts/ezw313

53. Digital reconstruction of the vitellointestinal duct and it's relations to the developing mesentery Olwyn R Conlon BSc, MBBS student [1], Kevin G Byrnes MBBS, PhD [1], Calvin J Coffey BSc, BMedSci, MB, PhD, AFRCSI, FRCSI [1], Dara Walsh BSc [1]

[1] Department of Surgery, University Hospital Limerick, St. Nessan's Road, Dooradoyle, Limerick, V94F858

Introduction: The vitellointestinal duct (VD) or omphalomesenteric duct (OMD) is an extension of the endodermal gut tube connecting the embryonic midgut to the extraembryonic yolk sac and remains patent from the fifth to the ninth gestational week. It is observed histologically at Carnegie Stage 13 (day 28-35). This study aimed to elucidate the embryological course and development of the VD and determine whether the VD, like abdominal digestive organs such as the intestines, liver, spleen and pancreas develops within primitive mesentery.

Methods: Data in the form of digitized histological cross-sectional embryological sections from Carnegie Stage 13 were obtained with the permission of Dr Kevin Byrnes. Within the aligned data set, manual tracing highlighted mesodermal and endodermal anatomical regions of interest. Image analysis software (Cinema4D; Maxon Computer GmbH, Germany) generated a 4-dimensional model exploring the course of the VD through the mesodermal mesentery.

Results: Computer rendered reconstruction of the VD established the duct to be surrounded by mesenteric mesoderm along its entire length. Three-dimensional exploratory videos were rendered of the mapped areas of interest.

Conclusion: It is evident from our 3D reconstructions of embryonic mesenteric midgut structures that the VD develops within ventral mesodermal mesentery along its entire length. Primitive mesentery may promote growth and development of the VD supporting its role as a communicating tract between the yolk sac and embryonic mid gut. This finding is consistent with previous studies identifying ventral and dorsal mesenteric regions as providing a mesodermal platform within which abdominal digestive organs such as the intestines, liver, spleen and pancreas can develop. 
UNDERGRADUATE RESEARCH IN NATURAL AND CLINICAL SCIENCE AND TECHNOLOGY (URNCST) JOURNAL Read more URNCST Journal articles and submit your own today at: https://www.urncst.com

\author{
54. A pilot of simulation based training programme for Renal Unit Staff in the Royal Free Hospital Group \\ Colm O'Brien MB, BCh, BAO Student [1], Áine Burns MD, FRCP, MSc, Med Ed [2, 3] \\ [1] Undergraduate Medical Student, Royal College of Surgeons in Ireland, Dublin, Ireland \\ [2] Professor of Postgraduate Education, University College London, London, United Kingdom, WC1E 6DE \\ [3] Consultant Nephrologist, Royal Free Hospital Group, London, United Kingdom, NW3 2QG
}

Introduction: High fidelity mannequin simulation based training is a growing area, used throughout undergraduate and post graduate medical training. Recent studies have shown the benefits of simulation based for medical professionals of all levels, as it allows for the detection of procedural and caregiver failings, in a safe and risk- free environment [1].

The basis for this pilot was to follow an externally developed, pre-prescribed curriculum [2] for high fidelity simulation based training, in relation to medical emergencies that can occur during haemodialysis, and attempting to apply this curriculum within the Royal Free Hospital Group.

Methods: The pilot simulation took place with the use of a Laerdal 3G Sim Man, high fidelity mannequin, under the guidance of senior University College London Simulation and Medical Tutors. It was run in accordance with the UCL Simulation Teaching Guidelines, in the UCL Simulation Education Centre in the Royal Free Hospital. The simulation scenario involved tunnelled line sepsis for a haemodialysis patient, and the progression of management over a 25 minute period. A 20 minute debrief session took place following the scenario, to allow for breakdown of the scenario, and questionnaire completion.

Results: The pilot simulation took place with the participation of one Foundation Year 2 Doctor, one Core Medical Trainees (SHO) and two Clinical Fellow Doctors. Participants all responded favourably, both verbally and on paper, in relation to the effectiveness of the simulation in improving their knowledge, and perceived confidence, in dealing with medical emergencies in haemodialysis. There were also issues in relation to the structure of the simulation scenarios, and equipment used, which did not match up with the clinical set up in RFH.

Conclusion: Although this was a single pilot scenario that was carried out, the simulation identified a clear desire for further simulation based practices within RFH. Simulation based scenarios such as the above trialled curriculum have been implemented formally into renal specialist training within the Royal Free Hospital Group, with further studies to take place over the coming months.

\title{
References
}

[1] Gordon JA, Wilkerson WM, Shaffew DW, Armstrong EG. Practicing Medicine without risk: Students' and educators' response to high-fidelity patient simulation. Acad Med. 2001 May; 76(5): 469-72. http://doi.org/10.1097/00001888-200105000-00019

[2] Watson K, Calvert S, Lasoye T, Rankin A. In Situ Simulation Team Training for the Management of Medical Emergencies occurring on haemodialysis: a practical guide. King's College Clinical Teaching. Accessed January 9th, 2019. Available from: https://renal.org/wp-content/uploads/2019/01/In-situ- haemodialysis-team-simulation-training-_A-practical-guide.pdf.

\author{
55. SOCS3 gene therapy prevents intimal hyperplasia and restenosis following balloon angioplasty in coronary artery \\ disease \\ Devendra K. Agrawal, M.Sc., Ph.D. (Biochemistry), Ph.D. (Medical Sciences), MBA, MS (ITM), FAAAAI, FAHA, FAPS, \\ FIACS [1], Victoria E. D. Wilson, MSc, Medical Student [2] \\ [1] Senior Vice President for Research \& Biotechnology; Professor, Department of Translational Research, \\ Western University of Health Sciences, Pomona, California, United States of America, 91766 \\ [2] School of Medicine, Dentistry, and Biomedical Sciences, Queen's University Belfast, Belfast, County Antrim, \\ Northern Ireland BT9 7BL
}

Introduction: Neointimal hyperplasia and restenosis following coronary balloon angioplasty is a significant problem. Noting absence of SOCS3 genes in neointima and inflamed sites, we predicted decreased SOCS3 expression post-angioplasty results in uncontrolled atherogenic cytokine-induced inflammation. We examined SOCS3 gene therapy effects on intimal hyperplasia and restenosis following intervention and determined underlying mechanisms.

Methods: Yucatan microswine consumed a high cholesterol diet for 24 weeks. Serum lipids, complete metabolic panel, and blood counts were regularly measured. Animals were subjected to angioplasty and intravascular stenting. The pAAV2/9-SM22SOCS3-GFP or pAAV2/ 9-SM22-PTEN-GFP vector delivered SOCS3 or PTEN genes to the procedural site, whereas controls received pAAV2/9-SM22-GFP vector. After 20-24 weeks, stenosis was examined by angiography, optical coherence tomography, histopathology, and immunohistochemistry for inflammatory markers. Complete blood profile and inflammatory markers evaluated potential vector immunogenicity. Epigenetically-induced effector proteins possibly involved in neointimal hyperplasia were examined. Angioplasty and intravascular stenting initially increased arterial lumen; however, at 20-24 weeks, significant intimal hyperplasia and restenosis developed.

Shaw et al. | URNCST Journal (2020): Volume 4, Issue 3

DOI Link: https://doi.org/10.26685/urnest.182 
UNDERGRADUATE RESEARCH IN NATURAL AND CLINICAL SCIENCE AND TECHNOLOGY (URNCST) JOURNAL Read more URNCST Journal articles and submit your own today at: https://www.urncst.com

Results: SOCS3 transgene significantly attenuated intimal hyperplasia and restenosis following angioplasty together with decreased TNF- and IGF-1. No significant hematology differences occurred pre- or post-coronary intervention with AAV2/9, nor did AAV2/9 \pm SOCS3 affect plasma fibrinogen, serum amylase, or C-reactive protein. DNMT1 appears the major epigenetic regulator, as it was differentially induced in intimal hyperplasia lesions post-angioplasty. AAV2/9 did not induce systemic inflammatory or immune responses.

Conclusion: Findings support potential application of SOCS3 gene therapy to prevent intimal hyperplasia and restenosis of coronary arteries following balloon angioplasty.

56. Evaluating the effect of ethnicity on dysglycemia and markers of type 2 diabetes risk in postpartum women with previous gestational diabetes mellitus Onyi Maduekwe, MB, BCh, BAO Student [1, 2], Stephanie Read, PhD [2], Lorraine Lipscombe, MD, MSc [2, 3]

[1] Department of Medicine, Queen's University Belfast, Belfast, Northern Ireland

[2] Division of Endocrinology, Department of Medicine, Women's College Hospital, Toronto, ON, Canada

[3] Division of Endocrinology and Metabolism, Department of Medicine, University of Toronto, Toronto, ON, Canada

Introduction: The prevalence of gestational diabetes mellitus (GDM) has increased substantially in developed countries. Postpartum dysglycemia persists in one third of women with GDM [1] and one in five will develop type 2 diabetes (T2DM) within 5 to 10 years of childbirth, which varies by ethnicity [2]. This study investigated the effect of ethnicity on postpartum dysglycemia and the role of T2DM risk factors on that association among women with recent GDM.

Methods: This cross-sectional study evaluated baseline characteristics from a pilot trial of a T2DM prevention program in women with recent GDM. We compared various T2DM risk factors such as BMI and waist circumference, across ethnicity. We then compared likelihood of dysglycemia between non-Caucasian (Afro-Caribbean, South Asian, Southeast Asian, other) and Caucasian women, using logistic regression.

Results: Compared to Caucasian women, South Asian women had a lower waist circumference $(83.8 \mathrm{vs} .95 .8 \mathrm{~cm}, \mathrm{P}<0.0001)$; and Southeast Asian women had a lower BMI (26.8 vs. $\left.31.3 \mathrm{~kg} / \mathrm{m}^{2}, \mathrm{P}<0.022\right)$. Overall $30.8 \%$ of postpartum women had dysglycemia, with higher rates in non-Caucasian women vs. Caucasian. After adjustment, Southeast Asian and South Asian women were significantly more likely to have dysglycemia than Caucasian women (adjusted odds ratios, aOR: 6.18 [95\% confidence interval, CI 1.81, 23.68] and 3.16 [1.01, 10.68] respectively).

Conclusion: South Asian and Southeast Asian women tended to have a lower burden of T2DM risk factors but were more likely to have postpartum dysglycemia than Caucasian women. This highlights a population of women with GDM who may be at higher risk for T2DM without traditional risk factors.

\section{References}

[1] Benhalima K, Jegers K, Devlieger R, Verhaeghe J, Mathieu C. Glucose Intolerance after a Recent History of Gestational Diabetes Based on the 2013 WHO Criteria. PLoS ONE. 2016; 11(6): e0157272. http://doi.org/10.1371/journal.pone.0157272

[2] Kousta E, Efstathiadou Z, Lawrence NJ, et al. The impact of ethnicity on glucose regulation and the metabolic syndrome following gestation diabetes. Diabetologia. 2006 Jan; 49(1): 36-40. http://doi.org/10.1007/s00125-005-0058-6

\section{A cadaveric study on lung fissure variations}

Conor Sheahan, MB, BCh, BAO Student [1], Gerges Abdelsayed [1], Harumi Ramanayake [1], Cara Connolly [1]

[1] Department of Anatomy and Regenerative medicine, The Royal college of Surgeons in Ireland (RCSI), Dublin, Ireland

Introduction: The purpose of this study was to observe variances in lung fissures within an Irish Cohort.

Methods: In adult cadavers, paired lungs were examined. A general inspection of the lungs was performed: Lung fissures were measured and aberrant fissures recorded. Fissures were graded by the 'Craig Walker' classification whereby Grade 1 is a complete fissure, Grade 2 is complete in length with parenchymal fusion at the lung base, grade 3 is incomplete by length and grade 4 is a total fusion. Exclusion criteria included the presence of lung malignancy, localized fibrosis, or previous lobectomy. Results: 28 lungs (14 pairs) were examined in 5 males and 9 females with a mean age of 82. Results are given as Mean $+/-$ S.D in males and females respectively: Grade 1 \& 2 right oblique fissures (ROF) were $33.00 \mathrm{~cm}+/-$ S.D 4.3 and $29.3 \mathrm{~cm}+/-$ S.D $6.8 \mathrm{~cm}$. Grade $1 \& 2$ Right Transverse Fissures (RTT) were $20.6 \mathrm{~cm}+/-$ S.D $3.5 \mathrm{~cm}$ and $18.3 \mathrm{~cm}+/-$ S.D 1.6 . The grade $1 \& 2$ left oblique fissures (LOF) were $32.3 \mathrm{~cm}+/-$ S.D $6.2 \mathrm{~cm}$ and $33.7 \mathrm{~cm}+/-$ S.D 5.01. No statistical difference between male and female findings.

(Students TTest) 
UNDERGRADUATE RESEARCH IN NATURAL AND CLINICAL SCIENCE AND TECHNOLOGY (URNCST) JOURNAL Read more URNCST Journal articles and submit your own today at: https://www.urncst.com

Of the 14 pairs of lungs examined, incomplete fissures (Grade 3) were identified in 1 ROF, 1 LOF and 6 RTF. Only one fissure was completely absent (grade 4); a LOF. Aberrant fissures were identified on 2 right lungs and 4 left.

Conclusion: Variable lung fissure morphology was more typical than standard lung fissures descriptions. We believe that these findings are clinically and anatomically significant as they can inform both surgical techniques and the interpretation of radiological images.

58. Dentate gyrus volume is associated with memory performance in pediatric-onset multiple sclerosis Nilaksa Sivanenthiran, MBBS Student [1] Giulia Longoni, MD [2, 3]

[1] University College Dublin, Dublin, Ireland

[2] University of Toronto, Toronto, Ontario, Canada

[3] Hospital for Sick Children, Toronto, Ontario, Canada

Introduction: Selective disruption of the dentate gyrus (DG) subfield of the hippocampus has been associated with memory impairment in mice at the early stage of experimental multiple sclerosis (MS) [1]. Such relationship has not been investigated in humans. This study aims to investigate the association between DG volume (DGv) and episodic memory in pediatric-onset MS (poMS) patients.

Methods: 15 poMS patients and $28 \mathrm{HC}$ were included. Participants underwent standardized research MRI and completed 3 episodic memory sub-tests included in the Penn Computerized Neurocognitive Battery. Composite memory (CM) scores for accuracy or reaction time (RT) were obtained by averaging scores from the 3 sub-tests. The DG was automatically segmented and manually edited using dedicated softwares. The association between DGv and memory scores were modelled in each group using generalized linear models (GLMs) adjusting for age, sex, and intracranial volume. Results were Bonferroni adjusted for multiple comparisons.

Results: DGv did not differ between groups $\left(\mathrm{HC}+9.2 \mathrm{~mm}^{3} v s\right.$ poMS, $\left.\mathrm{p}=0.7\right)$. CM-RT and nDGv were positively associated in the poMS group $\left(68 \mathrm{~mm}^{3} /\right.$ unit-increase of MC-RT score, $\left.\mathrm{p}=0.003\right)$. HC experienced smaller changes of DGv with increasing memory compared to poMS $\left(-6.8 \mathrm{~mm}^{3} \mathrm{DGv} / \mathrm{unit}\right.$-increase of MC-RT score, $\left.p=0.0011\right)$.

Conclusion: DGv is positively associated with CM-RT in youth with poMS. Reasons for weaker association in HC are unclear, although possibly explained by a decreased functional reserve with higher dependence of memory performance on hippocampal integrity in poMS. Future steps involve increasing sample size and continuing to investigate the association of memory outcomes with all hippocampal subfields.

\title{
Reference
}

[1] Planche V, Panatier A, Hiba B, Ducourneau EG, Raffard G, Dubourdieu N, et al. Selective dentate gyrus disruption causes memory impairment at the early stage of experimental multiple sclerosis. Brain Behav Immun. 2017 Feb; 60: $240-254$. https://doi.org/10.1016/j.bbi.2016.11.010

\author{
59. Paediatric Liaison Psychiatry Services (PLPS) at Our Lady's Children's Hospital Crumlin (OLCHC) - Who are \\ they seeing? \\ Alix B Petit, BSc, GEM Student [1], Fiona McNicholas, Professor [1, 2] \\ [1] UCD School of Medicine, University College Dublin, Belfield, Dublin 4, Ireland \\ [2] Department of Child and Adolescent Psychiatry, Our Lady's Children's Hospital, Crumlin, Dublin 12, Ireland
}

Introduction: Paediatric Liaison Psychiatry Services (PLPS) is a specialized tertiary service aimed at addressing co-morbid mental health $(\mathrm{MH})$ difficulties with medical illnesses.

Methods: Prior audits conducted in 2016 and 2018 showed that the majority of cases seen by PLPS were acute MH presentations to the emergency department (ED) as opposed to the classic liaison cases [1]. A six-month retrospective audit was conducted using anonymized referral letters of all children and adolescents who presented to PLPS (N=118) in January-June 2019.

Results: Patients presenting ranged from 5-18 $(\mathrm{M}=13.6, \mathrm{SD}=2.53)$ and predominantly female $(67,57 \%)$. The majority of cases were acute psychiatry presentations to the $\operatorname{ED}(75,66 \%), 26(23 \%)$ were referred by college paediatricians as true liaison cases, $5(4 \%)$ were eating disorder cases, and $8(7 \%)$ were seen as outpatients. $90(80 \%)$ had an Axis 1 diagnosis, most commonly depression (30\%), ADHD (32\%), Anxiety (14\%). Of acute MH presentations to the ED, 45\% (34) presented out of hours, $51 \%$ (38) were admitted with a mean length stay 4.9 days. 62\% (69) of patient were referred to Child and Adolescent Mental Health Services (CAMHS), 6\% (7) to a specialized inpatient psychiatry unit, and 26\% (29) back to the medical team/GP.

Shaw et al. | URNCST Journal (2020): Volume 4, Issue 3

DOI Link: https://doi.org/10.26685/urnest.182 
UNDERGRADUATE RESEARCH IN NATURAL AND CLINICAL SCIENCE AND TECHNOLOGY (URNCST) JOURNAL Read more URNCST Journal articles and submit your own today at: https://www.urncst.com

Conclusion: The majority of the cases seen by the PLPS were acute MH presentation to the ED, with a smaller focus on true liaison cases. Of these, $43 \%$ (31) were previously known to CAHMS. Sourcing an emergency and 'out of hours' CAMHS service is supported by this audit.

\section{Reference}

[1] Lynch F, Kehoe C, MacMahon S, McCarra E, McKenna R, D'Alton A et al. Paediatric Consultation Liaison Psychiatry Services (PCLPS) -what are they actually doing?. Irish Medical Journal. 2017 Dec; 110(10): 652.

60. Gestational folate and folic acid intake across five countries: A secondary analysis of FACT Elaine G. Rose, MB, BCh, BAO Student, MSc [1, 2], Malia S.Q. Murphy, PhD [1], Katherine A. Muldoon, PhD [1], Alysha L.J. Harvey, MSc [1], Ruth Rennicks White, RN, BScN [1, 3], Shi Wu Wen, PhD [1, 3-5], Mark C. Walker, MD, FRCPC, $\mathrm{MSc}, \mathrm{MScHCM}[1,3-6]$

[1] OMNI Research Group, Clinical Epidemiology Program, Ottawa Hospital Research Institute, Ottawa, Ontario, Canada [2] School of Medicine and Medical Science, University College Dublin, Belfield, Dublin, Ireland

[3] Department of Obstetrics, Gynecology \& Newborn Care, The Ottawa Hospital, Ottawa, Ontario, Canada

[4] Department of Obstetrics and Gynecology, University of Ottawa, Ottawa, Ontario, Canada

[5] School of Epidemiology and Public Health, University of Ottawa, Ottawa, Ontario, Canada

[6] BORN Ontario, Children's Hospital of Eastern Ontario, Ottawa, Ontario, Canada

Introduction: Folic acid supplementation is widely recommended to prevent fetal neural tube defects. The objective of this study was to evaluate differences in dietary food folate intake and folic acid supplementation amongst pregnant women in Canada, Australia, Argentina, Jamaica and the United Kingdom (UK).

Methods: The study was nested in FACT [1], an international multi-centre, randomized, double-blinded, placebo- controlled, phase III trial investigating folic acid for the prevention of pre-eclampsia in high-risk pregnancies. Participants completed the Block dietary folate equivalent (DFE) screener at 8-16 weeks of gestation. Differences in natural food folate intake, early gestational folic acid supplementation and total DFE were assessed. Statistical associations were assessed by Kruskal-Wallis rank-sum tests for continuous data, and chi-square test or fisher's exact test for categorical data.

Results: 2440 women completed DFE screener surveys. 81.4\% (n=1987) participants reported taking folic acid supplements. Folic acid intake from supplements was highest in Canada (med:1000 ug/day, IQR:1000-1000) and lowest in Argentina (med:150 ug/day, IQR:0-1000). Natural food folate intake was highest in Argentina (med:154.7 ug/day, IQR:106.1-198.0) and lowest in the UK (med:118.1ug/day, IQR:84.0-157.6). Total DFE levels were as follows: Canada (med:1835.7ug/day, IQR:1783.7-1897.1), Jamaica (med:1159.8 ug/day, IQR:300.7-1486.6), Australia (med:1056.37 ug/day, IQR:892.4-1480.3), UK (med:769.1ug/day, IQR:160.4-821.5) and Argentina (med:452.5ug/day, IQR:163.3-1833.5).

Conclusion: The majority of women in our cohort received above recommended daily levels of folate intake (400 ug/day). Our data do not consider national folic acid fortification programs in Canada, Australia and Argentina. Given that many women exceeded the upper tolerable limit of folate (1000 ug/day) from natural food sources and supplements alone, re-examination of common supplementation practices is warranted.

\section{Reference}

[1] Wen S. W., Rennicks White R., Rybak N, Gaudet L. M., Robson S, Hague W., et al. Effect of high dose folic acid supplementation in pregnancy on pre-eclampsia (FACT): double blind, phase III, randomised controlled, international, multicentre trial. BMJ Clinical Research. 2018 Sep: 362, k3478. https://doi.org/10.1136/bmj.k3478 
UNDERGRADUATE RESEARCH IN NATURAL AND CLINICAL SCIENCE AND TECHNOLOGY (URNCST) JOURNAL

Read more URNCST Journal articles and submit your own today at: https://www.urncst.com

61. Neuroinflammation and neuroplasticity in the spinal cord and substantia nigra following chronic hypercapnia in goats

Raghad Alshammasi., MB, BCh, BAO Student [1], Hubert M. Forester, PhD [2, 3]

[1] UCD School of Medicine, University College Dublin, Belfield, Dublin 4, Ireland

[2] Department of Physiology, Medical College of Wisconsin.

[3] Neuroscience Research Center, Medical College of Wisconsin, Milwaukee, WI 53226.

Introduction: Hypercapnic patients in respiratory disease have worse prognoses than those with equal impairment without it. Time-dependent physiologic effects of chronic hypercapnia that correspond to glutamatergic and inflammatory changes in the brainstem respiratory nuclei (BRN) have recently been characterized [1]. Elevated steady-state ventilation was observed, during 30 days of chronic hypercapnia in goats; due to an unknown stimulus [2]. The aims of this research were to explore the involvement of: (1) the spinal cord; being the major output to the muscles of respiration, (2) the substantia nigra; a dopaminergic population of neurons that is connected to the retrotrapezoid nucleus (RTN) through the periaqueductal grey (PAG).

Methods: Tissue was obtained from healthy goats after 24 hours or 30 days of chronic exposure to $6 \%$ In $\mathrm{CO}_{2}$ or room air. Spinal cords and brains were rapidly extracted and flash frozen for western blot analysis to assess inflammation and glutamate receptor expression.

Results: In the spinal cord, results show no significant differences between hypercapnic and control goats, neither at 24 hours, nor at 30 days. However, in the substantia nigra, results show a $60 \%$ decrease of the AMPA receptor subunit GluR2 after 24 hours, that returns to near control levels at 30 days; it is also apparent that there is a $52 \%$ increase of the inflammation marker IL $1 \beta$ at 30 days.

Conclusion: Damage to AMPA receptors indicates decreased excitability and connectivity. An increase of inflammation at 30 days is unusual, and suggests long-term rewiring within the substantia nigra that may contribute to the concomitant physiologic changes that occur during chronic hypercapnia.

62. The perioperative management of nonvalvular atrial fibrillation patients on long-term oral anticoagulation therapy: A dilemma for GPs (Systematic Review) Aisling Egan, Medical Student [1], Peter Hayes, General Practitioner [2]

[1] Graduate Entry Medical Student, University of Limerick, Ireland

[2] Senior Clinical Tutor in General Practice, University of Limerick, Ireland

Introduction: The aim of this systematic review was to assess the perioperative management of nonvalvular atrial fibrillation (AF) patients on long-term oral anticoagulation therapy in a Primary Care setting.

Methods: A comprehensive search strategy was performed on varying databases including, EMBASE, Pubmed, NIH clinical trials and Cochrane database of systematic reviews for clinical trials, cohort studies, randomised control trials, meta-analysis, systematic reviews relating to 'perioperative management', 'Nonvalvular Atrial fibrillation', 'bridging', 'Primary Care', 'General Practitioner' and 'anticoagulation'. The identified studies from the literature search were then further evaluated using our strict inclusion and exclusion criteria. The Critical Appraisal Skills Programme (CASP) tools ('CASP Qualitative assessment.' (2017), Available at: https://casp-uk.net/casp-tools-checklists) and Cochrane database of systematic reviews was used for critical appraisal and evaluate for possible bias of the selected papers [1].

Results: Studies analysed, in this systematic review, suggest that further research is warranted to investigate perioperative management of nonvalvular AF patients on long-term anticoagulation therapy in a Primary Care setting. From the results gathered, it could be argued that GPs should consider an individualised approach to the administration of treatment for these patients' optimal perioperative care.

Conclusion: Standardised protocols and larger studies may help to further evaluate the clinical significance of patient and procedure specific perioperative management of nonvalvular AF patients on long-term oral anticoagulation therapy in a Primary Care setting.

\section{Reference}

[1] CASP Qualitative assessment. in (2017). Available at: https://casp-uk.net/casp-tools-checklists 
UNDERGRADUATE RESEARCH IN NATURAL AND CLINICAL SCIENCE AND TECHNOLOGY (URNCST) JOURNAL

Read more URNCST Journal articles and submit your own today at: https://www.urncst.com

63. Clinical characteristics of IPF patients: A ten year single centre experience

Dalia Abualsaud, MB, BCh, BAO [1], Alan Kelly, B.A. Mod Biochem, MBBS [1, 2] Carita Bramhill, MPH [1], Michael Keane, MB, BCh, BAO, MRCPI, MD [1, 2], Cormac McCarthy, MD, PhD, MRCPI [1, 2]

[1] UCD School of Medicine, University College Dublin, Dublin, Belfield Dublin 4, Ireland

[2] Department of Respiratory Medicine, St. Vincent's University Hospital Dublin, Dublin 4, Ireland

Introduction: Idiopathic pulmonary fibrosis (IPF) is a chronic progressive lung disease with significant mortality and it is essential to understand the natural progression of this condition.

Methods: The project aimed to describe the clinical characteristics of an Irish IPF population. Data was retrieved from St. Vincent's University Hospital ILD registry for 87 IPF patients enrolled from 2009-2017 and 202 patient/physician encounters in the ILD clinic were assessed prospectively [1].

Results: Mean age of diagnosis was 69 years and the prominent symptoms were dyspnoea $(72 \%, \mathrm{n}=52)$ and cough $(60 \%, \mathrm{n}=43)$. $37 \%$ of all patients had undergone lung biopsy, however, this decreased from 39\% prior to 2013 to 30\% between 2013-2017. The annual rates of decline for FVC and DLCO were $114 \mathrm{ml} \pm 157 \mathrm{ml}$ and $7 \% \pm 7.7 \%$, respectively. Patients were grouped according to the Gender-Age-Physiology index (Table 1). Of 202 physician encounters, 49\% ( $\mathrm{n}=99$ ) did not document cough as a queried symptom or its absence. Leicester cough questionnaire demonstrated that cough was not particularly impactful on quality of life in this population (mean LCQ 16/21).

Table 1: Patient Stages.

\begin{tabular}{|l|l|}
\hline Stage & Percentage/frequency \\
\hline 1 & $23(39 \%)$ \\
\hline 2 & $25(42 \%)$ \\
\hline 3 & $11(19 \%)$ \\
\hline
\end{tabular}

Conclusion: While biopsy rates were high, there was a slight decrease over time. Most patients were diagnosed at stage 2, requiring close monitoring. IPF is a progressive lung disease, cough is a common presenting symptom, however in this group it appears to be poorly correlated with quality of life. To better understand the importance of cough, it is important to address it in all consultations.

\section{Reference}

[1] Cho PSP, Birring SS, Fletcher HV, Turner RD. Methods of Cough Assessment. J Allergy Clin Immunol Pract. 2019

Jul - Aug; 7(6): 1715-1723. https://doi.org/10.1016/j.jaip.2019.01.049

64. The relationship of athlete factors and patient reported outcomes on return to play 1-year post-ACL reconstruction

Liana Balaghi, MB, BCh, BAO Student [1], Enda King, PhD [2], Lindsay Tetreault, PhD [1],

Eanna Falvey, MB, BCh, PhD, MRCPI, MmedSci (SEM), FFSEM [1,2]

[1] School of Medicine, University College Cork, Cork, Ireland

[2] Sports Surgery Clinic, Dublin, Ireland

Introduction: Anterior cruciate ligament (ACL) tears are the most frequently reported knee injury in athletes. For those who wish to return to play (RTP), ACL reconstruction (ACLR) is recommended to restore knee function and stability. Knowledge of important predictors of RTP post-ACLR can aid surgeons and allied health professionals to effectively manage athletes' rehabilitation expectations. The purpose of this study was to determine which athlete factors and patient reported outcomes predict RTP at 1-year post-ACLR.

Methods: This prospective cohort study recruited 336 participants who underwent ACLR at SSC in Dublin. Data collected included; baseline demographics, details of sport participation, injury mechanism, intent to RTP and patient-reported outcome measure (PROM) questionnaires at baseline (Marx, ACL-RSI) and at 1-year post-ACLR (Marx, ACL-RSI, Cincinnati, IKDC). Participants were categorised based on successful RTP 1-year post-ACLR. Univariate and multivariate logistic regression analysis were used to evaluate the association between RTP and athlete factors and PROMs. 
UNDERGRADUATE RESEARCH IN NATURAL AND CLINICAL SCIENCE AND TECHNOLOGY (URNCST) JOURNAL
Read more URNCST Journal articles and submit your own today at: $\underline{\text { ttps://www.urncst.com }}$

Results: Two hundred twenty (65.45\%) participants RTP at 1-year post-ACLR. Participants were more likely to RTP if they were younger (OR:0.972, 95\%CI:0.952-0.995), intended to return to a higher level of sport (OR:2.125, 95\%CI:1.169-3.861), had higher baseline Marx scores (OR: 1.066, 95\%CI:1.022-1.111), and higher Marx (OR:1.291, 95\%CI:1.214-1.373) and IKDC scores at 1-year postoperative (OR:1.065, 95\%CI:1.041-1.088). Higher Marx scores at 1-year postoperative (OR:1.291, 95\%CI:1.214-1.373) were the only significant predictors of RTP.

Conclusion: Patients were more likely to RTP if they had better functional activity outcomes at 1-year post-ACLR, suggesting that PROMs can be used to assess how likely an athlete is to RTP.

\section{Low falls causing major injury: A retrospective cohort study}

Kathryn A. Lesko, BSc, MHS, MB, BAO, BCH student [1],

Conor Deasy, MB, Dip IMC, Dip Tox, DCH, FCEM, FACEM, PhD [1, 2]

[1] School of Medicine, University College Cork, Cork, Ireland T12 X838

[2] Consultant in Emergency Medicine, Cork University Hospital, Wilton, Cork, Ireland

Introduction: Falling from a height of under 2 meters is one of the most common presentations to the emergency department [1]. This presentation encompasses a wide patient cohort, from paediatric sport injuries to elderly falls. Our aim is to characterise this entire patient population and its various sub-populations, to identify preventative strategies and care pathways to improve outcomes for patients.

Methods: We used the Trauma Audit and Research Network (TARN) database [2] to retrospectively identify patients presenting to the Cork University Hospital Emergency Department with trauma resulting from a fall of under 2 meters from January 2015 to June 2018

Results: The database returned 1066 qualifying cases, with a mean age of 67.3 years $(\mathrm{SD}=21)$ and a median age of 71.3 years $(\mathrm{IQR}=23) ; 44 \%$ were male. Mechanical falls accounted for $\mathrm{n}=513(48 \%)$ of low fall injuries, followed by stationary falls $\mathrm{n}=265$ $(25 \%)$. Injuries occurred most often at home $n=515(48 \%)$, followed by public places $n=208(19.5 \%)$. The most severely injured body region was limbs $n=526(49.3 \%)$, followed by head $n=253(23.7 \%)$. The number of patients with Glasgow Outcome Scores of 4 (moderate disability) and 5 (good recovery) were $n=488(45.8 \%)$, and $n=390(36.6 \%)$ respectively. There were 79 deaths in the cohort.

Conclusion: This cohort represent middle-age to elderly (55-85) population who suffer significant morbidity as a result of their injuries. Wait times, length of stay, and mortality increase with age. Mechanical falls at home are the most common cause of low-fall major trauma.

\section{References}

[1] National Office of Clinical Audit. Major Trauma Audit National Report 2017. National Office of Clinical Audit 2019.

[2] National Office of Clinical Audit. The Trauma Audit Research Network (TARN). Available from: tarn.ac.uk 
UNDERGRADUATE RESEARCH IN NATURAL AND CLINICAL SCIENCE AND TECHNOLOGY (URNCST) JOURNAL Read more URNCST Journal articles and submit your own today at: https://www.urncst.com

Table 1: Demographics.

\begin{tabular}{|c|c|c|c|c|c|}
\hline & Paeds $(<18)$ & Adult (18-74.9) & Elderly (75+) & Total & p test \\
\hline $\mathrm{n}$ & 52 & 546 & 468 & 1066 & \\
\hline Sex $=$ Male $(\%)$ & $32(61.5)$ & $\overline{281(51.5)}$ & $156(33.3)$ & $4469(44.0)$ & 40.001 \\
\hline Description (\%) & & & & & $<0.001$ \\
\hline Stationary fall & $2(3.8)$ & $118(21.6)$ & $145(31.0)$ & $265(24.9)$ & \\
\hline Mechanical fall & $10(19.2)$ & $252(46.2)$ & $251(53.6)$ & $513(48.1)$ & \\
\hline Athletic fall & $9(17.3)$ & $5(0.9)$ & $1(0.2)$ & $15(1.4)$ & \\
\hline Fall down stairs & $1(1.9)$ & $62(11.4)$ & $36(7.7)$ & $99(9.3)$ & \\
\hline Fall up stairs & $1(1.9)$ & $4(0.7)$ & $6(1.3)$ & $99(9.3)$ & \\
\hline External Forces fall & $3(5.8)$ & $26(4.8)$ & $2(0.4)$ & $31(2.9)$ & \\
\hline Impact fall & $23(44.2)$ & $48(8.8)$ & $10(2.1)$ & $81(7.6)$ & \\
\hline Seizure-induced & $1(1.9)$ & $18(3.3)$ & $1(0.2)$ & $20(1.9)$ & \\
\hline Unknown Mechanism & $2(3.8)$ & $13(2.4)$ & $16(3.4)$ & $31(2.9)$ & \\
\hline Location (\%) & & & & & $<0.001$ \\
\hline Home & $16(30.8)$ & $247(45.2)$ & $252(53.8)$ & $515(48.3)$ & \\
\hline Assisted Living & $0(0.0)$ & $7(1.3)$ & $33(7.1)$ & $40(3.8)$ & \\
\hline Hospital & $0(0.0)$ & $8(1.5)$ & $7(1.5)$ & $15(1.4)$ & \\
\hline Public & $20(38.5)$ & $122(22.3)$ & $66(14.1)$ & $208(19.5)$ & \\
\hline Sports Arena & $6(11.5)$ & $25(4.6)$ & $0(0.0)$ & $31(2.9)$ & \\
\hline Workplace & $2(3.8)$ & $22(4.0)$ & $4(0.9)$ & $28(2.6)$ & \\
\hline Not Specified & $8(15.4)$ & $115(21.1)$ & $106(22.6)$ & $229(21.5)$ & \\
\hline Mode of arrival (\%) & & & & & $<0.001$ \\
\hline Aircraft & $0(0.0)$ & $0(0.0)$ & $1(0.2)$ & $1(0.1)$ & \\
\hline Ambulance & $22(43.1)$ & $391(75.2)$ & $388(87.0)$ & $801(78.8)$ & \\
\hline Ambulance and helicopter & $1(2.0)$ & $2(0.4)$ & $2(0.4)$ & $5(0.5)$ & \\
\hline Car / personal vehicle & $25(49.0)$ & $72(13.8)$ & $27(6.1)$ & $124(12.2)$ & \\
\hline Helicopter & $0(0.0)$ & $3(0.6)$ & $1(0.2)$ & $4(0.4)$ & \\
\hline Walking & $1(2.0)$ & $21(4.0)$ & $11(2.5)$ & $33(3.2)$ & \\
\hline Not applicable & $2(3.9)$ & $25(4.8)$ & $15(3.4)$ & $42(4.1)$ & \\
\hline Other & $0(0.0)$ & $6(1.2)$ & $1(0.2)$ & $7(0.7)$ & \\
\hline Wait Times (median [IQR]) & $\begin{array}{r}40[14.25, \\
81.5] \\
\end{array}$ & $81[32,153.5]$ & $\begin{array}{r}95[42.25, \\
173.75]\end{array}$ & $82[36,155]$ & $<0.001$ \\
\hline GCS (median [IQR]) & $\begin{array}{r}15.00[15.00, \\
15.00]\end{array}$ & $\begin{array}{r}15.00[15.00, \\
15.00]\end{array}$ & $\begin{array}{r}15.00[15.00 \\
15.00]\end{array}$ & $\begin{array}{r}15.00[15.00, \\
15.00]\end{array}$ & $\begin{array}{r}0.080 \\
\text { nonnorm }\end{array}$ \\
\hline GCS Range (\%) & & & & & 0.149 \\
\hline Mild (13-15) & $47(90.4)$ & $486(89.0)$ & $433(92.5)$ & $966(90.6)$ & \\
\hline Moderate (9-12) & $0(0.0)$ & $23(4.2)$ & $9(1.9)$ & $32(3.0)$ & \\
\hline Severe (3-8) & $2(3.8)$ & $22(4.0)$ & $12(2.6)$ & $36(3.4)$ & \\
\hline Not Recorded & $3(5.8)$ & $15(2.7)$ & $14(3.0)$ & $32(3.0)$ & \\
\hline ISS (median [IQR]) & $\begin{array}{r}9.00[9.00, \\
17.00] \\
\end{array}$ & $\begin{array}{r}9.00[9.00, \\
16.00] \\
\end{array}$ & $\begin{array}{r}9.00[5.00, \\
16.00] \\
\end{array}$ & $\begin{array}{r}9.00[9.00, \\
16.00] \\
\end{array}$ & $\begin{array}{r}0.068 \\
\text { nonnorm } \\
\end{array}$ \\
\hline ISS band $(\%)$ & & & & & $<0.001$ \\
\hline $1-8$ & $6(11.5)$ & $88(16.1)$ & $125(26.7)$ & $219(20.5)$ & \\
\hline $9-15$ & $27(51.9)$ & $301(55.1)$ & $208(44.4)$ & $536(50.3)$ & \\
\hline$>15$ & $19(36.5)$ & $157(28.8)$ & $135(28.8)$ & $311(29.2)$ & \\
\hline Intubation $=$ Yes $(\%)$ & $1(1.9)$ & $36(6.6)$ & $12(2.6)$ & $49(4.6)$ & 0.006 \\
\hline Most severely injured body region $(\%)$ & & & & & $<0.001$ \\
\hline Limbs & $27(51.9)$ & $286(52.4)$ & $213(45.5)$ & $526(49.3)$ & \\
\hline Head & $19(36.5)$ & $122(22.3)$ & $112(23.9)$ & $253(23.7)$ & \\
\hline Spine & $1(1.9)$ & $52(9.5)$ & $60(12.8)$ & $113(10.6)$ & \\
\hline Chest & $1(1.9)$ & $47(8.6)$ & $49(10.5)$ & $97(9.1)$ & \\
\hline Multiple & $0(0.0)$ & $25(4.6)$ & $26(5.6)$ & $51(4.8)$ & \\
\hline Face & $0(0.0)$ & $10(1.8)$ & $7(1.5)$ & $17(1.6)$ & \\
\hline Abdomen & $4(7.7)$ & $4(0.7)$ & $0(0.0)$ & $8(0.8)$ & \\
\hline Other & $0(0.0)$ & $0(0.0)$ & $1(0.2)$ & $1(0.1)$ & \\
\hline LOS (median [IQR]) & $\begin{array}{r}4.00[3.00, \\
5.25] \\
\end{array}$ & $\begin{array}{r}8.00[5.00, \\
16.00]\end{array}$ & $\begin{array}{r}12.00[6.00, \\
23.00]\end{array}$ & $\begin{array}{r}9.00[5.00, \\
19.00] \\
\end{array}$ & $\begin{array}{r}<0.001 \\
\text { nonnorm }\end{array}$ \\
\hline Outcome at 30 days $=$ Dead $(\%)$ & $0(0.0)$ & $28(5.1)$ & $43(9.2)$ & $71(6.7)$ & 0.005 \\
\hline \multicolumn{6}{|l|}{ Comorbidities } \\
\hline Hypertension $=$ Yes $(\%)$ & $0(0.0)$ & $151(27.7)$ & $271(57.9)$ & $422(39.6)$ & $<0.001$ \\
\hline Hypercholesterolaemia = Yes $(\%)$ & $0(0.0)$ & $99(18.1)$ & $132(28.2)$ & $231(21.7)$ & $<0.001$ \\
\hline Osteoporosis = Yes (\%) & $3(5.8)$ & $54(9.9)$ & $88(18.8)$ & $145(13.6)$ & $<0.001$ \\
\hline Alcohol abuse $=$ Yes $(\%)$ & $0(0.0)$ & $115(21.1)$ & $29(6.2)$ & $144(13.5)$ & $<0.001$ \\
\hline Depression $=$ Yes $(\%)$ & $0(0.0)$ & $73(13.4)$ & $53(11.3)$ & $126(11.8)$ & 0.015 \\
\hline
\end{tabular}


UNDERGRADUATE RESEARCH IN NATURAL AND CLINICAL SCIENCE AND TECHNOLOGY (URNCST) JOURNAL

Read more URNCST Journal articles and submit your own today at: https://www.urncst.com

66. The use of fibrinogen concentrate (FC) in an acute obstetric setting at Cork University Maternity Hospital (CUMH)

Jun Marc Feng MB, BCh, BAO Student [1], Connie Foley [2], Greg O'Conner MSc [3], John Sheehy MSc [3],

Mary R. Cahill MB, BCh, BAO, $M D$ [4]

[1] School of Medicine, University College Cork, Cork, Ireland

[2] Cork University Maternity Hospital, Cork, Ireland

[3] Cork University Hospital Blood Transfusion Laboratory, Cork, Ireland

[4] Department of Haematology, Cork University Hospital, Cork, Ireland

Introduction: Before 23/07/14, FC was transported from CUH transfusion laboratory to CUMH theatres during an obstetric hemorrhage $(\mathrm{OH})$. The policy was amended to place an emergency stock of FC in CUMH to save time and allow easier access to FC. This study aims to audit if FC in CUMH led to increased use during $\mathrm{OH}$, and to investigate the correlation between the use of FC with red cell product (RCC).

Methods: A study was conducted comparing patients that received FC in CUMH from 23/07/12 to 23/07/14 (control group) to the patients that received $\mathrm{FC}$ from 23/07/14 to 23/07/16 (case group) during $\mathrm{OH}$.

Results: There was a $6.12 \%$ increase in the use of FC from the control to the case group. Both groups showed a positive association between FC and RCC transfused. There was no statistically significant difference in the average fibrinogen administered per patient when comparing the two groups. The average amount of blood products (RCC, SDP and PLT) transfused per patient was lower in the case group. Also, the average pre- and post-treatment fibrinogen levels were higher in the case group.

Conclusion: This study showed that the change to place FC in CUMH coincided with increased use. However, the data demonstrated that as more FC was administered, the more blood products were used. Also, the case group showed a decrease in average blood products used per patient compared to the control group, which could suggest that in time, earlier FC use may lead to a further reduction in the use of blood products.

Table 1: Comparison of Average Fibrinogen Administered and Blood Products Transfused.

\begin{tabular}{|c|c|c|c|}
\hline & $\begin{array}{c}\text { Pre-Local } \\
\text { Availability Policy } \\
\text { Group (Control) }\end{array}$ & $\begin{array}{c}\text { Post-Local } \\
\text { Availability Policy } \\
\text { Group (Case) }\end{array}$ & P-Value \\
\hline Fibrinogen Administered (Units) per Patient & $2.50 \pm 2.014$ & $2.00 \pm 2.508$ & 0.133 \\
\hline RCC Transfused (Units) per Patient & $4.50 \pm 3.538$ & $3.00 \pm 2.873$ & $<0.05$ \\
\hline SDP Transfused (Units) per Patient & $2.00 \pm 2.687$ & $1.00 \pm 2.276$ & $<0.05$ \\
\hline PLT Transfused (Units) per Patient & $1.00 \pm 1.172$ & $0 \pm 0.903$ & $<0.05$ \\
\hline Total Blood Products Transfused (RCC+ SDP & $8.50 \pm 6.67$ & $4.0 \pm 5.506$ & $<0.05$ \\
\hline + PLT) per Patient (Units) & & $2.879 \pm 1.221$ & $<0.05$ \\
\hline Pre-Fibrinogen Level (g/L) per Patient & $1.3958 \pm 0.85541$ & $3.5 \pm 1.0563$ & $<0.05$ \\
\hline
\end{tabular}

67. Investigating phenotypic and metabolic profiles in rheumatoid arthritis macrophages and monocytes Jacob W. Gilchrist, BSc, MB, BCh, BAO (Hons) Student [1, 2]

[1] School of Medicine, Trinity College Dublin, the University of Dublin, Dublin 2, Ireland

[2] School of Medicine, University College Dublin, Belfield, Dublin 4, Ireland

Introduction: Rheumatoid Arthritis (RA) is a chronic autoimmune disease characterized by abnormal angiogenesis and leucocyte infiltration that stimulates erosion within the joint [1]. Macrophages form a vital component of the cellular infiltrate, however their specific functional phenotype has yet to be fully elucidated [2]. The aim of this study therefore was to assess if phenotypic differences in diseased and healthy monocytes and macrophages exist. 
UNDERGRADUATE RESEARCH IN NATURAL AND CLINICAL SCIENCE AND TECHNOLOGY (URNCST) JOURNAL Read more URNCST Journal articles and submit your own today at: https://www.urncst.com

Methods: RA and Healthy Control (HC) monocytes were isolated from peripheral blood samples, with macrophages derived from monocyte sample stimulation. Expression of M1 and M2 macrophage-associated gene markers was assessed in monocytes and M0 macrophages using RT-PCR. Phagocytic capacities of monocytes and M0, M1 and M2 macrophages was quantified using FITC-conjugated ovalbumin flow cytometry. Expression of glycolysis-associated proteins (GLUT1, HK2, PFKFB3, pSTAT3) was assessed in RA M0, M1 and M2 macrophages and HC M0 macrophages using western blotting and densitometry. Results: RA monocytes and M0 macrophages exhibited similar expression levels of M1 macrophage-associated genes, but decreased expression levels of M2 macrophage-associated genes compared to HC samples. RA monocytes showed an enhanced phagocytic response to LPS-stimulation compared to HC populations. Finally, RA M1 macrophages demonstrated increased expression of HK2, PFKFB3 and pSTAT3 compared to RA M0 and M2 macrophages, and increased expression of HK2 and PFKFB3 compared to HC M0 macrophages.

Conclusion: RA monocytes and macrophages show an increased phagocytic and glycolytic phenotype, which is paralleled by a decreased resolving capacity. Thus, infiltrating macrophages are implicated as vital drivers of inflammation and damage within the RA joint.

\section{References}

[1] Fearon U, Hanlon MM, Wade SM, Fletcher JM. Altered metabolic pathways regulate synovial inflammation in rheumatoid arthritis. Clin Exp Immunol. 2019 Aug; 197(2): 170-180. https://doi.org/10.1111/cei.13228

[2] Veale, D.J., Orr, C. \& Fearon, U. Cellular and molecular perspectives in rheumatoid arthritis. Semin Immunopathol. 2017; 39: 343-354. https://doi.org/10.1007/s00281-017-0633-1w

\section{Paramedical perceptions of end of life care in Ireland}

S. Murphy, MB, BCh, BAO Student [1], J. Van der Velde, MB, BCh, CAO [1, 2]

[1] School of Medicine, University College Cork, Ireland

[2] Emergency Department, Cork University Hospital, Cork, Ireland

Introduction: Critical decisions made in the field by paramedical practitioners influence where patients die, if their end of life (EOL) wishes are upheld and how appropriately health-care resources are used. Despite this, paramedical services rarely have practice guidelines or protocols that address the unique challenges presented by patients at EOL. This study aims to explore current practice, identify if there is a rural/urban variation in current practice and gauge perceptions amongst paramedical staff as to their future role in enhancing EOL care in Ireland.

Methods: A qualitative approach was employed to collect data through two focus group interviews. A convenience sample of fifteen practitioners (group $1 \mathrm{n}=7$, group $2 \mathrm{n}=8$ ) took part in this study. Focus groups were audio recorded, transcribed and analysed using Attride-Stirling's framework for thematic network analysis.

Results: The global theme 'Paramedical Perceptions of Their Role in End of Life Care' emerged from five organising themes: 1. education and training; 2. current clinical practice guidelines; 3. communication; 4. environment and 5. staff support. Poor communication between those involved in patient care, lack of support from current clinical practice guidelines, limited training in managing EOL scenarios and inadequate staff supports were highlighted by participants. The clinical environment also effected how challenging practitioners found the call.

Conclusion: The pathway to improving EOL care must include an emphasis on improvements in practitioner education and training, enhanced communication between all those involved in a patient's care and offering non didactic practice guidelines that are practitioner driven and patient-focused. It must also include increased psychological supports for paramedical practitioners dealing with EOL patients.

69. Bowel preparation for inpatient colonoscopy at Naas General Hospital: Audit of adherence to gastrointestinal endoscopy national quality improvement programme guidelines Sarah-Jane O'Connor [1], Dr. Mohamed Elsiddig [2], Alice Whelan [1], Dr. Ion Cretu [2]

[1] Graduate Entry Medical School, University of Limerick, Limerick, Ireland

[2] Department of Gastroenterology, Naas General Hospital, Naas, Co. Kildare W91 AE76, Ireland

Introduction: It has been documented that inpatient colonoscopy can be more challenging than outpatient colonoscopy, with poorer quality of bowel preparation and reduced rates of successful completion of the procedure. This audit aimed to investigate the quality of bowel preparation for, and success rate of, inpatient colonoscopy at Naas General Hospital.

Shaw et al. | URNCST Journal (2020): Volume 4, Issue 3

DOI Link: https://doi.org/10.26685/urnest.182 
UNDERGRADUATE RESEARCH IN NATURAL AND CLINICAL SCIENCE AND TECHNOLOGY (URNCST) JOURNAL Read more URNCST Journal articles and submit your own today at: https://www.urncst.com

Methods: All patients undergoing inpatient colonoscopy at Naas General Hospital between $1^{\text {st }}$ June 2018 and $1^{\text {st }}$ June 2019 were identified retrospectively. Endoscopy reports were obtained using EndoRAAD software. Successful colonoscopy was defined as intubation of the caecum with satisfactory bowel preparation as per "Guidelines for the Implementation of a National Quality Improvement Programme in GI Endoscopy" [1]. A further group of patients undergoing outpatient colonoscopy during the same time period were identified for comparison purposes.

Results: 112 individuals were identified as having undergone inpatient colonoscopy, representing $5.4 \%$ of all procedures performed during this period. One-third (33\%) were completed due to 'unexplained anaemia' and 15\% due to 'rectal bleeding', with several other indications comprising the remainder. Only 49.1\% (55/112) of inpatient bowel preparation was rated by the endoscopist as satisfactory [2], compared with $90 \%$ of outpatient preparation for the same period.

Conclusion: This audit demonstrated that the quality of inpatient colonoscopy falls short of the recommended standard. The reasons for this are likely complex and multifactorial but may include reduced mobility and poorer adherence to bowel preparation and oral hydration. Deferring colonoscopy until after discharge from hospital should therefore be considered whenever the indication for the procedure allows.

\section{References}

[1] Working Group GI Endoscopy National QI Programme, Conjoint Board of RCPI \& RCSI. Guidelines for the Implementation of a National Quality Improvement Programme in GI Endoscopy - Version 5.2 (2017).

[2] Enns R, Krygier D. The inpatient colonoscopy: A difficult endeavour. Can J Gastroenterol. 2008 Dec; 22(11): 900-2. https://doi.org/10.1155/2008/826271

70. Retrospective assessment of blood culture sampling practice in a regional Paediatric Emergency Department Patricia Bacus [1], Deirdre Foley [2], Patrick Stapleton [3], Elizabeth Mahony [2]

[1] Graduate Entry Medical School, University of Limerick, Limerick, Ireland

[2] Department of Pediatric, University Hospital of Limerick, Limerick, Ireland

[3] Department of Microbiology, University Hospital of Limerick, Limerick, Ireland

Introduction: Blood culture (BC) sampling can be a challenging process in paediatrics. Cultures are often taken without correct indications and using a sub-standard technique. We wish to assess compliance with correct indications for blood culture sampling, results of cultures taken and subsequent management of same.

Methods: We obtained records of all blood culture results sampled over a four week period in September and October 2019. We included all patients attending our paediatric Emergency Department (ED) who were reviewed by a paediatric medical doctor. We cross-referenced $\mathrm{BC}$ records and results with patient attendance notes, to determine what proportion of the cultures were taken under correct indications.

Results: A total of 110 blood cultures were tested during the study period. Only 21 patients (19\%) had a fever of 38 degrees Celsius or higher at time of assessment. 59\% of our patients sampled were tachycardic at time of assessment (referenced by age-appropriate PEWS charts). $86 \%$ of patients had no documentation of skin appearance on presentation, with the remaining patients described as 'mottled' $(n=1)$, 'pale' $(n=1)$, 'non-blanching' $(n=3)$, 'blanching rash' $(n=9)$ and 'pink' (n=1). 27\% had clinically significant indications for blood cultures documented in notes $(n=30)$. This included suspected sepsis $(n=5)$, urinary tract infection $(n=8)$, and suspected meningitis $(n=4)$ amongst others. The remaining 80 patients sampled had no documented reason for BC sampling. $10(9.1 \%)$ of $\mathrm{BC}$ samples grew bacteria consistent with contamination from skin or mouth flora. 32 patients $(29 \%)$ were started on antibiotics

Conclusion: Blood culture sampling technique is poor, and this is evident from our high rates of contaminant growth in culture bottles $(9.1 \%)$. Blood culture samples being taken where no indication is found. This results in important patient-care and cost implications. We require further guidelines on indications for blood culture sampling, and education on correct sampling technique in our paediatric ED. 
UNDERGRADUATE RESEARCH IN NATURAL AND CLINICAL SCIENCE AND TECHNOLOGY (URNCST) JOURNAL Read more URNCST Journal articles and submit your own today at: https://www.urncst.com

71. An exploration of patients' knowledge and anxiety associated with incidentally found pulmonary nodules and development of an Irish Thoracic Society endorsed national patient information leaflet for pulmonary nodules Jessica McCarthy, Medical Student [1], Aisling Farrell, Respiratory SpR [2], John Alickolli, Specialist Respiratory Nurse [3], Oisin O'Connell, Respiratory Consultant [3]

[1] Department of Medicine, University College Cork, Co Cork, Ireland

[2] Department of Respiratory Medicine, Cork University Hospital, Co Cork, Ireland

[3] Department of Respiratory Medicine, Bon Secours Hospital, Co Cork, Ireland

Introduction: Pulmonary nodules are common incidental findings on CT imaging. The primary aim of this study is to identify patient's understanding, anxiety and perceived cancer risk of pulmonary nodules. The second aim is to develop a national patient information leaflet on pulmonary nodules.

Methods: A cross-sectional survey of patients who were under active surveillance for pulmonary nodules was carried out in a private hospital. 60 patients were analysed to evaluate patients' level of understanding, concerns and anxiety surrounding nodules. A patient information leaflet was developed with input from the 8 Irish Cancer Centres of excellence to address patient concerns.

Results: $73 \%$ patient response rate to study (44/60). Mean age $64.20 .5 \%$ experienced clinically significant distress on diagnosis of a pulmonary nodule, higher amongst female patients $(p<0.05)$. Those with significant distress estimated their perceived risk of lung cancer to be $13 \%$, those who were not significantly distressed estimated their risk to be $8 \%(\mathrm{p}<0.05)$. Knowledge gaps surrounding the natural history and need for surveillance were identified. These knowledge gaps and patient questions were answered in the patient information leaflet.

Conclusion: Pulmonary nodules are a significant cause of patient distress, with inadequate patient understanding surrounding pulmonary nodules. The implementation of an Irish Thoracic Society endorsed pulmonary nodule patient information leaflet will help bridge knowledge gaps and alleviate distress.

72. Quality improvement study in hypertension managed in primary care Shivani Bhat, MPH [1], Mary Murphy [2], David Molony BMBS [3]

[1] Graduate Entry Medical School, University of Limerick, Limerick, Ireland

[2] The 4th Practice, Cardiology, Mallow Primary Healthcare Centre, Mallow, Ireland

[3] The Red House Clinic, Mallow Primary Healthcare Centre, Mallow, Ireland

Introduction: Elevated BP was the leading global contributor to premature death accounting for more than 10 million deaths. $24 \mathrm{hr}$ ambulatory blood pressure monitoring (ABPM) has emerged as the gold standard in establishing the presence of hypertension disease. We investigate the current state of play in conducting clinic BP and 24 ABPM to determine future hypertension management in primary care patients at the Red House Clinic.

Methods: Retrospective cross-sectional chart audit from November 2016 to 2019 was conducted where patients who had a latest clinical BP systolic $>150 \mathrm{mmHg}$ or a diagnosis of hypertension were included in the analysis. Output variables included current $\mathrm{BP}$ reading, latest 24ABPM readings, patient demographics, presence of cardiovascular related co-morbidities and cardiac medication intake.

Results: Off a total of 951 patient searched, 928 were included in analysis where the mean age was 64.2 years, $42 \%$ were females, mean BP was $145 / 80 \mathrm{mmHg}$, mean BMI 23.8 and $53 \%$ of the patients had a 24ABPM reading. Based on the latest clinic BP reading, $19 \%$ fell into the high normal category of hypertension, $41 \%$ grade $1 \mathrm{HTN}$, and $17 \%$ grade $2 \mathrm{HTN}$. More than $70 \%$ of the patients had a coded diagnosis of hypertension and $45 \%$ of the patients were on an ACE inhibitor or ARB. Off the patients with a BP reading 140/90 - 159/99 $\mathrm{mmHg}, 53 \%$ had a 24ABPM.

Conclusion: This chart audit reveals a considerable gap in hypertension management where only $53 \%$ of the patients with a $\mathrm{BP}$ systolic $\mathrm{BP}>150 \mathrm{mmHg}$ had a $24 \mathrm{ABPM}$ reading. To increase the $24 \mathrm{ABPM}$ to determine hypertension management, we have designed a hypertension protocol to be standardized across the Mallow Primary Health Centre to ensure adequate referral for ABPM, follow-up and drug efficacy.

Shaw et al. | URNCST Journal (2020): Volume 4, Issue 3

DOI Link: https://doi.org/10.26685/urnest.182 
UNDERGRADUATE RESEARCH IN NATURAL AND CLINICAL SCIENCE AND TECHNOLOGY (URNCST) JOURNAL Read more URNCST Journal articles and submit your own today at: https://www.urncst.com

73. Multimodal retinal imaging of autosomal dominant PRPH2-associated retinitis pigmentosa

Ethan Waisberg, MB, BCH, BAO student [1, 3], Michalis Georgiou, MD [1, 2] and Michel Michaelides MD, PhD [1, 2]

[1] UCL Institute of Ophthalmology, University College London, 11-43 Bath Street, London, EC1V 9EL, UK

[2] Moorfields Eye Hospital NHS Foundation Trust, City Road, London, EC1V 2PD, UK

[3] UCD School of Medicine, University College Dublin, Belfield, Dublin 4, Ireland

Introduction: To present the retinal imaging findings of a patient with retinitis pigmentosa.

Methods: Clinical investigation was performed including ophthalmologic examination, electroretinography (ERG), and retinal imaging with optical coherence tomography (OCT), fundus autofluorescence (FAF) and adaptive optics scanning light ophthalmoscopy (AOSLO). Sanger sequencing was used to screen genes with variants associated with retinal degeneration.

Results: The patient was severely affected, with reduced visual acuity and constricted visual field. AOSLO imaging revealed irregularities in the photoreceptor mosaic. OCT imaging depicts ellipsoid zone loss and FAF shows areas of atrophy of the retinal pigment epithelium.

Conclusion: Herein we characterize a case of a novel PRPH2 variant causing autosomal dominant retinitis pigmentosa, and we describe the disruption of the retinal structure. AOSLO imaging can be used effectively to analyse abnormalities in the photoreceptor mosaic.

74. Cognitive impairment and dementia in parkinsonian syndromes

Kimberly ZX. Tan, Medical Student [1], Suzanne Timmons, MD, MB, BCh, BAO, MSc [1, 2]

[1] School of Medicine, University College of Cork, Cork, Munster, Ireland

[2] Centre for Gerontology and Rehabilitation, Saint Finbarr's Hospital, Cork, Munster, Ireland

Introduction: As PD progresses, cognitive impairment develops in both iPD and aPD. The latter includes PSP, MSA, CBD. Deterioration of PDMCI to PDD affects daily independence [1]. Although the MoCA is recommended for cognitive assessment, it is not routinely administered [2]. Furthermore, the UPDRS, has only one cognitive self-report item. Thus, much PDMCI and PDD is undetected. We aim describe the prevalence of PD-MCI and PDD in PD Syndromes.

Methods: A retrospective cohort study $(\mathrm{n}=152)$ of iPD and aPD patients aged $\geq 60$ years-old, attending a geriatrician-provided Movement Disorder clinic, was performed. The prevalence and incidence of clicician diagnosed PD-MCI and PDD; scores and frequency of MoCA and UPDRS (total and motor sub-scores) were collected from chart reviews.

Results: Overall, there were 143 iPD and 9 aPD patients. Of which, 85 had or developed PDMCI (56\%) and 37 had or developed PDD (24\%) over a median follow-up of around 2 years. At first clinic review, a small number already had PDMCI (7 iPD and $1 \mathrm{aPD}$ ). PDMCI and PDD prevalence increased with time, with peak incidence of PDMCI and PDD in iPD being around 4 and 6 years from symptom onset respectively. At first clinic review, worse cognitive MoCA score, had a weakmoderate correlation with worse UPDRS motor score $(\mathrm{p}=0.012)$ and total score $(\mathrm{p}=0.014)$.

Conclusion: As PD progresses, patients develop PDMCI, and around 1/4 developed PDD by a median of around 2 years since symptom onset. Cognitive scores are associated with PD motor scores, but not age. Regular cognitive assessment is warranted in elderly with PD.

\section{References}

[1] Diagnostic and statistical manual of mental disorders. 5th ed. Arlington, Va.: American Psychiatric Association; 2013.

[2] Nilsson FM. Mini mental state examination (MMSE)-probably one of the most cited papers in health science. Acta Psychiatrica Scandinavica. 2007 Aug; 116(2): 156-7. https://doi.org/10.1111/j.1600-0447.2007.01037.x 
UNDERGRADUATE RESEARCH IN NATURAL AND CLINICAL SCIENCE AND TECHNOLOGY (URNCST) JOURNAL

Read more URNCST Journal articles and submit your own today at: https://www.urncst.com

\section{Effects of $\mathrm{CD}^{4+}$ and $\mathrm{CD}^{8+} \mathrm{T}$-cell subsets on changes in the $\mathrm{CD}^{4}: \mathrm{CD}^{8}$ ratio in ambulatory ART-treated people living with HIV}

Emmanuel I. Ohaa, MPH [1], Alan Macken, PhD [2], Gerard Sheehan, PhD [2, 3], John S. Lambert, PhD [1-3], Evan G. Muldoon, PhD [2], Aoife G. Cotter, PhD [2, 3], Patrick W. Mallon, PhD [1-3], Willard Tinago, PhD [2]. [1] School of Medicine, University College Dublin, Belfield, Dublin 4, Ireland

[2] Centre for Experimental Pathogen-Host Research, University College Dublin, Belfield, Dublin 4, Ireland

[3] Department of Infectious Disease, Mater Misericordiae University Hospital, Dublin 7, Ireland

Introduction: Immune recovery on effective antiretroviral therapy (ART) is likely a composite of initial redistribution of trapped memory $\mathrm{CD}^{4+}$ and $\mathrm{CD}^{8+} \mathrm{T}$-cells, accompanied by a continuous slow repopulation with newly produced naïve $\mathrm{T}$-cells. Associations between higher naïve T-cell subsets with higher $\mathrm{CD}^{4}: \mathrm{CD}^{8} \mathrm{~T}$-cell ratio have been demonstrated in cross-sectional analyses. This study aimed to examine if any T-cell subset predicted changes in $\mathrm{CD}^{4}: \mathrm{CD}^{8}$ ratio over time.

Methods: In an observational prospective cohort of people living with HIV (PLWH), data on $\mathrm{CD}^{4}: \mathrm{CD}^{8}$ ratio and expanded T-cell subsets (naïve $\left[\mathrm{CD} 45 \mathrm{RO}^{-} \mathrm{CD} 62 \mathrm{~L}^{+}\right]$, central memory $\left[\mathrm{CD} 45 \mathrm{RO}^{+} \mathrm{CD}^{2} \mathrm{~L}^{+}\right]$and effector memory $\left[\mathrm{CD}^{2} 5 \mathrm{RO}^{+} \mathrm{CD} 62 \mathrm{~L}^{-}\right]$) were evaluated using flow cytometry on fresh blood samples. Demographic and clinical data were collected from patients' records. Association between T-cell subsets measured at study entry and changes in $\mathrm{CD}^{4}: \mathrm{CD}^{8}$ ratio over follow-ups were assessed using linear mixed-effects models.

Results: 173 ART-treated subjects, age 43( \pm 9$)$ years, $63 \%$ male and 66\% Caucasian, were followed up for a median(IQR) of 6.4(5.9-6.6) years. 96(55\%) individuals maintained viral suppression throughout follow-up. In multivariable analyses adjusting for demographic, clinical and treatment-related factors, higher $\% \mathrm{CD}^{8+}$ naïve $(+0.012, \mathrm{p}=0.005)$ was associated with a higher $\mathrm{CD}^{4}: \mathrm{CD}^{8}$ ratio at cohort entry and lower subsequent increase $(-0.005, \mathrm{p}<0.001)$ in $\mathrm{CD}^{4}: \mathrm{CD}^{8}$ ratio over time. No significant effect was observed with other T-cell subsets.

Conclusion: $\mathrm{CD}^{8+}$ naïve T-cells were shown to be independently associated with changes in the $\mathrm{CD} 4: \mathrm{CD}^{8} \mathrm{~T}$-cell ratio in this cohort of PLWH and might have a potential role in determining which subjects would achieve greater improvements in their T-cell ratio.

76. Mental health psycho-education: Are we making the grade?

Friars D [1], Minihan E [2], McNicholas F [1-3]

[1] UCD School of Medicine, University College Dublin, Belfield, Dublin 4, Ireland

[2] Our Lady's Children's Hospital, Dublin 12, Ireland

[3] Lucena Clinic Services, Rathgar, Dublin 6, Ireland

Introduction: With mental health services under-resourced and underfunded in Ireland today, clinicians are under pressure to find innovative means of delivering care. Psychoeducation is an evidence based therapeutic intervention for patients and families, providing information and support to better cope with illness [1, 2]. In Child and Adolescent Mental Health Services (CAMHS), parent psychoeducation is offered alongside traditional interventions, and empowers families to support optimised mental wellbeing in their young person. Since 2006, parent psycho-education, along with clinician training has been an aim of the Lucena Foundation. The aim of this study is to evaluate recent psychoeducation events organised by Lucena CAMHS. The study will reveal aspects of the sessions which appeal to attendees and will shed light on some of their limitations.

Methods: At 7 events overall satisfaction, along with interest and relevance were rated on a Likert scale of 1-5. Three events were open to the public, while 4 were targeted at particular cohorts. Anonymous data were input into SPSS for analysis and descriptive and correlational statistics obtained. Qualitative feedback was also collected. 
UNDERGRADUATE RESEARCH IN NATURAL AND CLINICAL SCIENCE AND TECHNOLOGY (URNCST) JOURNAL Read more URNCST Journal articles and submit your own today at: https://www.urncst.com

\begin{tabular}{|l|l|l|l|l|l|l|l|}
\hline & $\begin{array}{l}\text { Eating } \\
\text { Disorder } \\
\text { Awareness } \\
\text { Evening }\end{array}$ & $\begin{array}{l}\text { TICS, } \\
\text { Tourette's } \\
\text { and OCD } \\
\text { Evening }\end{array}$ & $\begin{array}{l}\text { ADHD } \\
\text { Evening }\end{array}$ & $\begin{array}{l}\text { 22q Behaviour } \\
\text { Management } \\
\text { Drop-In }\end{array}$ & $\begin{array}{l}\text { ADHD } \\
\text { Masterclass }\end{array}$ & $\begin{array}{l}\text { Eating } \\
\text { Disorder } \\
\text { Masterclass }\end{array}$ & $\begin{array}{l}\text { Catatonia } \\
\text { Masterclass }\end{array}$ \\
\hline $\begin{array}{l}\text { Number of } \\
\text { respondents }\end{array}$ & 35 & 14 & 14 & 7 & 20 & 20 & 12 \\
\hline Type of event & $\begin{array}{l}\text { Public parents } \\
\text { evening }\end{array}$ & $\begin{array}{l}\text { Public } \\
\text { parents } \\
\text { evening }\end{array}$ & $\begin{array}{l}\text { Public } \\
\text { parents } \\
\text { evening }\end{array}$ & $\begin{array}{l}\text { Invite-only } \\
\text { parents afternoon }\end{array}$ & $\begin{array}{l}\text { Day-long } \\
\text { clinician } \\
\text { training }\end{array}$ & $\begin{array}{l}\text { Day-long } \\
\text { clinician } \\
\text { training }\end{array}$ & $\begin{array}{l}\text { Day-long } \\
\text { clinician } \\
\text { training }\end{array}$ \\
\hline Attendance Fee & Free & Free & Free & Free & $€ 100$ & $€ 100$ & $€ 100$ \\
\hline
\end{tabular}

Results: Response to all events was overwhelmingly positive, with high mean overall satisfaction scores (Parents $=4.36 /$ Clinicians $=4.46$ ). Most notably at the public Eating Disorder evening, there was a strong correlation (Pearson's $r=0.78, p=0.003$ ) between relevance of topic and overall satisfaction.

Conclusion: Psychoeducational events are being perceived by attendees as relevant and informative, with high satisfaction ratings. These preliminary results suggest that psychoeducation events are feasible, cost-effective and should be offered alongside traditional treatments.

\section{References}

[1] Dixon L, McFarlane WR, Lefley H, Lucksted A, Cohen M, Falloon I, Mueser K, Miklowitz D, Solomon P, Sondheimer D. Evidence-based practices for services to families of people with psychiatric disabilities. Psychiatr Serv. 2001 Jul; 52(7): 903-10. https://doi.org/10.1176/appi.ps.52.7.903

[2] Lukens EP, McFarlane WR. Psychoeducation as Evidence-Based Practice: Considerations for Practice, Research, and Policy. Brief Treatment \& Crisis Intervention. 2004 Oct 1; 4(3). https://doi.org/10.1093/brief-treatment/mhh019

\section{Do surgical department discharge letters meet the HIQA national standard? A clinical audit in South Tipperary General Hospital Bridgid Ferriter, BSc Student [1], Athar Noshad Sheikh, Consultant General Surgeon [2] \\ [1] Graduate Entry Medical School, University of Limerick, Limerick, Ireland [2] Department of Surgery, South Tipperary General Hospital, Clonmel, Tipperary, Ireland}

Introduction: This audit was performed due to a perceived lack of quality of discharge summaries in South Tipperary General Hospital (STGH). The quality of discharge summaries effects patient care through their effect on continuity of care, patient readmission and unnecessary presentation to primary care [1-3]. Importantly, this is the first pass at a subject that, due to its effect on quality patient care will only increase in importance as the system of prospective funding (previously "money follows the patient") becomes reality $[4,5]$.

Method: This clinical audit used the HSE Guide to Clinical Audit and the HIQA "National Standard for Patient Discharge Summary Information".

Results: Target performance of a sample of 102 was 100\%. The audit achieved an overall compliance result of $49 \%$.

Conclusion: Following this audit the authors recommend that important fields that have never been included in the audit template be added, that training be implemented and that consultants commit to providing correction and feedback. The improvement of surgical discharge summaries at STGH will be a cyclical process where multiple iterations will be completed and audited with the goal of reaching a GOLD Standard discharge planning process. 
UNDERGRADUATE RESEARCH IN NATURAL AND CLINICAL SCIENCE AND TECHNOLOGY (URNCST) JOURNAL Read more URNCST Journal articles and submit your own today at: https://www.urncst.com

\author{
78. Potassium supplementation audit: St. Columcilles Hospital \\ Cooney S MB, BCh, BAO, [1], Boland L MB, BCh, BAO, [1], Ryan M [2] \\ [1] UCD School of Medicine, University College Dublin, Belfield, Dublin 4, Ireland \\ [2] St. Columcille's Hospital, Loughlinstown, Co. Dublin, D18 E365, Ireland
}

Introduction: Hypokalaemia is a common electrolyte abnormality in the inpatient population due to a variety of underlying causes. It is a high-risk area in patient safety, mortality and morbidity if inappropriately managed.

Methods: We conducted a clinical audit of the Potassium supplementation practices in St Columcille's Hospital (SCH) and the adherence to the local pharmacy guidelines. We liaised with the SCH Pharmacy department and reviewed the local potassium supplementation and monitoring guidelines. We developed an audit proforma examining the key aspects of this practice. We then reviewed the active drug Kardex of all inpatients on a single day, in all inpatient wards. Each episode of new hypokalaemia which required new supplementation was included as a separate entry. We did this as we were examining the potassium supplementation prescribing and monitoring safety rather than pathology.

Results: 83 patients' Kardex were reviewed, of which there were 12 episodes of K+ supplementation. 6 of these episodes were mild hypokalaemia 3.0-3.4 K+ and 6 were moderate 2.5-2.9 K+. There were no cases of severe hypokalaemia. 6 were initially commenced on PO replacement, and 6 were started on IV replacement. 11 of 12 received the correct route of administration. 4 out of $12(33.3 \%)$ of the treatments were an incorrect dosage. These 4 doses were all subtherapeutic. 11 of 12 had their U\&E correctly monitored.

Conclusion: The management of the majority of cases of hypokalemia treatment adhered to the hospital guidelines. However, there was deviation from guidelines in 4/12 cases. Presentation of audit data to NCHDs is necessary with follow up completion of the cycle of audit.

\author{
79. Safety issues in paediatric emergency departments: A research study \\ Mairéad O'Donnell, BSc Student [1], Ronan O'Sullivan, PhD [2, 3] \\ [1] School of Medicine, University College Cork, Cork, Ireland T12 AK54 \\ [2] Consultant in Emergency Medicine/Paediatric Emergency Medicine, Bon Secours Hospital, Cork, Ireland T12 DV56 \\ [3] Director, Paediatric Emergency Research Unit (PERU), National Children's Research Centre, Dublin 12, Ireland
}

Introduction: Paediatric Emergency Departments (PEDs) are a breeding ground for errors (Crosskerry at al. 2011). In 2015, PERUKI (Paediatric Emergency Research UK and Ireland) identified patient safety as one of the top 20 research priorities of paediatric emergency medicine (PEM) (Hartshorn et al). In 2018, $21 \%$ of the 65,000 CUH ED presentations were children (HSE, 2019). It is therefore paramount that potential areas for patient harm are identified to facilitate risk reduction. The aim of this project is to identify the leading safety issues occurring in PEDs.

Methods: A qualitative methodology, employing focus groups, was employed. Two focus groups, facilitated by a Consultant in PEM, were held, comprised of medical and nursing staff from a mixed adult/paediatric ED, and a tertiary-level paediatriconly ED, Interviews were conducted from a setlist of open and closed questions, audio-recorded, transcribed and analysed using the Attride-Stirling framework for thematic analysis.

Results: From the global theme of 'Safety Issues within PEDs' emerged the themes of: Staffing, Space and Support. The endless stop-gap solutions that become long-term 'fixes' in EDs are daily struggles identified by the participants - with patient's needs always taking precedence.

Conclusion: Safety issues are easily identified by staff in the ED. There are persistent endeavours to identify and reduce the potential for patient harm, through ongoing training, the implementation of new policies and persistent patient focused care to ensure the best patient outcome.

80. A five-year retrospective review of fatalities involving novel psychoactive substances in Southern Ireland Andrew Mazurek, HBSc, MB, BCh, BAO Student [1], Margot Bolster, MB, ChB, BAO, BA [2]

[1] School of Medicine, University College Cork, Cork, Ireland

[2] Department of Pathology, University College Cork, Cork, Ireland

Introduction: Novel Psychoactive Substances (NPS) are rapidly emerging and being reformulated to evade legislative controls and drug screens, creating unpredictability in drug markets and ineffective drug policies [1]. The National Advisory Committee on Drugs has recommended surveillance of local trends given that Ireland has the highest self-reported NPS use in Europe and literature regarding health risks is lacking [2]. The aim of the study was to elucidate demographic and autopsy findings of fatal cases involving NPS in Southern Ireland as compared to traditional drugs of abuse (TDOA).

Shaw et al. | URNCST Journal (2020): Volume 4, Issue 3

DOI Link: https://doi.org/10.26685/urnest.182 
UNDERGRADUATE RESEARCH IN NATURAL AND CLINICAL SCIENCE AND TECHNOLOGY (URNCST) JOURNAL Read more URNCST Journal articles and submit your own today at: https://www.urncst.com

Methods: Postmortem reports completed by the Assistant State Pathologist between 2012-2016 with positive toxicology for illicit substances were retrospectively analyzed to compare toxicology, pathology, and circumstances surrounding death between NPS and TDOA.

Results: Of 164 cases with illicit substances detected, 17 (10.4\%) involved NPS and in 64.7\% of those cases NPS contributed to the cause of death. Demographic characteristics were similar between NPS and TDOA as fatal abusers were predominantly male and young (mean 30 years). Importantly, cases involving NPS showed a higher proportion of mono-drug intoxication and presentation to hospital prior to death. Autopsy findings were minimal but commonly featured pulmonary congestion, aspiration of foreign material, and cerebral oedema.

Conclusion: NPS are involved in a small fraction of autopsy cases but contribute significantly to acute intoxication fatalities. Knowledge of common autopsy findings and associated demographics can reinforce the need for toxicological analysis when autopsy fails to find significant natural disease. Implementation of urine dipstick screens which include NPS is recommended to improve recognition of NPS overdoses presenting to hospital.

Table 1: Demographic Characteristics of Fatal Abusers (NPS vs TDOA).

\begin{tabular}{|l|c|c|}
\hline & NPS & TDOA \\
\hline Number of reported deaths & 11 & $89.1 \%$ \\
\hline Male gender & $90.9 \%$ & $32.1 \pm 8.26$ \\
\hline Age at death, mean \pm SD & $29.5 \pm 8.16$ & $59.4 \%$ \\
\hline Place of death & & $14.1 \%$ \\
\hline Home & $63.6 \%$ & $4.7 \%$ \\
\hline Hospital & $\mathbf{3 6 . 4 \%}$ & $35.9 \%$ \\
\hline Outdoor & $0 \%$ & $12.5 \%$ \\
\hline Known drug addiction & $37.5 \%$ & $\mathbf{2 7 . 3 \%}$ \\
\hline Index Drug on its Own & & \\
\hline
\end{tabular}

\section{References}

[1] Hohmann N, Mikus G, Czock D. Effects and risks associated with novel psychoactive substances: Mislabeling and sale as bath salts, spice, and research chemicals. Dtsch Arztebl Int. 2014 Feb; 111(9): 139-47. https://doi.org/10.3238/arztebl.2014.0139

[2] Kelleher C, Christie R, Lalor K, Fox J, Bowden M, O'Donnell C. An overview of new psychoactive substances and the outlets supplying them. Dublin: National Advisory Committee on Drugs, 2011.

\section{MSC-derived extracellular vesicles in an in vitro model of ventilator induced lung injury}

Daniel O'Toole PhD [1], Claire Masterson PhD [1], Shahd Horie PhD [1], Seán McCarthy PhD [1], Hector Gonzalez BSc [1], John Laffey MD [1], Victor Omiwole [2]

[1] Discipline of Anaesthesia, School of Medicine, NUIG

[2] College of Medicine, Nursing \& Health Science, NUIG

Introduction: Mesenchymal stromal cells (MSCs) are self-renewing, can undergo differentiation and secrete extracellular vesicles (EVs) among other factors. EVs reduce inflammation in lungs and could potentially treat Ventilator Induced Lung Injury (VILI) induced Acute Respiratory Distress Syndrome (ARDS). The aim was to determine if delivery of MSC derived EVs to the lung epithelium is a viable strategy for treatment of VILI.

Methods: BEAS2B lung epithelial cells on flexible tissue cultures plates received vehicle or control/human umbilical cord MSC derived EVs. Wells received 23\% biaxial stretch by Flexcell BioFlex Tension System for up to 8h and were returned to incubator overnight. LDH assay measured membrane integrity. MTT assay measured cell metabolism. ELISA determined IL-6 and IL-8 inflammatory cytokine concentrations. Results were analysed by t-test with P-values <0.05 considered significant. 
UNDERGRADUATE RESEARCH IN NATURAL AND CLINICAL SCIENCE AND TECHNOLOGY (URNCST) JOURNAL
Read more URNCST Journal articles and submit your own today at: $\underline{\text { ttps://www.urncst.com }}$

Results: IL-6, but not IL-8, secretion increased after $4 \mathrm{~h}$ stretch compared to resting controls $(8053.8 \pm 329.4 \mathrm{v} 7154.6 \pm 288.4$ $\mathrm{pg} / \mathrm{mL})$. IL-8 secretion increased after $8 \mathrm{~h}$ stretch $(665.88 \pm 73.09 \mathrm{v} 446.61 \pm 15.50 \mathrm{pg} / \mathrm{mL})$ and could be ameliorated by EV addition $(665.88 \pm 73.09$ v $527.83 \pm 14.89 \mathrm{pg} / \mathrm{mL})$. $8 \mathrm{~h}$ stretch decreased MTT viability compared to resting control $(91.16 \pm 2.88 \%$ v $100 \pm 2.59 \%)$ and was reversed by EV treatment though not significantly $(91.16 \pm 2.88 \%$ v $101.90 \pm 10.43 \%)$. No significant changes in $\mathrm{LDH}$ release were observed at $4 \mathrm{~h}$ or $8 \mathrm{~h}$ stretch. No toxicity or inflammation was observed in EV groups in resting controls.

Conclusion: EV treatment reversed cyclic stretch induced inflammation in lung epithelial cells and trended towards reversing cell injury, indicating a possible application in VILI patients after advancement to pre-clinical testing.

\section{Conflicts of Interest}

The authors declare that they have no conflict of interests.

\section{Authors' Contributions}

LS: Served as a planning committee member for the conference, invited speakers for the conference, organised the judging panel for the conference, assisted authors with their abstract, drafted the conference abstract booklet, and gave final approval of the version to be published.

SP: Served as a planning committee member for the conference, invited speakers for the conference, assisted authors with their abstract submissions, drafted the conference abstract booklet, and gave final approval for the version to be published.

NQ: Treasurer of the UCD Medical Society, negotiated sponsorship deals, assisted with the running of the conference, gave final approval for the abstract book to be published.

\section{Acknowledgements}

The Student Medical Summit National Committee: assisting in promoting and attracting students across Ireland to participate in the Student Medical Summit, acting as ambassadors to their respected colleges.

In alphabetical order:

Colm O'Brien- Royal College of Surgeons in Ireland

Christina Stevenson- University of Limerick

Glen O’Brien- University College Cork

Padraig Scully- NUI Galway

Peter Jackson- University College Cork

Sara Sotirakos- Trinity College Dublin

Sophie Mananian- Queen's University Belfast

Susie O’Neill- Trinity College Dublin

Thank you to all the judges and the UCD Medical Society for their contributions and diligent help on the day, especially to those listed below who have helped throughout the year.

Professor Donal O'Shea- Head Judge and advisor to the planning committee

Professor Marguerite Clyne- Head Judge

\section{Funding}

Bank of Ireland

University College Dublin School of Medicine

University College Dublin Societies Council

Irish Medical Times and MIMS Ireland

Direct Medics

UCD Medical Society 
UNDERGRADUATE RESEARCH IN NATURAL AND CLINICAL SCIENCE AND TECHNOLOGY (URNCST) JOURNAL Read more URNCST Journal articles and submit your own today at: https://www.urncst.com

\section{Article Information}

Managing Editor: Jeremy Y. Ng

Peer Reviewers: The abstracts were peer-reviewed by the Student Medical Summit's planning committee.

Article Dates: Received Feb 13 20; Published Mar 1720

\section{Citation}

Please cite this article as follows:

Shaw L, Pan X, Quirke N. Conference Abstract Booklet: 3rd Student Medical Summit 2020. 2020 Mar 17: 4(3).

https://urncst.com/index.php/urncst/article/view/182

DOI Link: https://doi.org/10.26685/urncst.182

\section{Copyright}

() Liam Shaw, Xiaoshan Pan, Ned Quirke. (2020). Published first in the Undergraduate Research in Natural and Clinical Science and Technology (URNCST) Journal. This is an open access article distributed under the terms of the Creative Commons Attribution License (https://creativecommons.org/licenses/by/4.0/), which permits unrestricted use, distribution, and reproduction in any medium, provided the original work, first published in the Undergraduate Research in Natural and Clinical Science and Technology (URNCST) Journal, is properly cited. The complete bibliographic information, a link to the original publication on http://www.urncst.com, as well as this copyright and license information must be included.

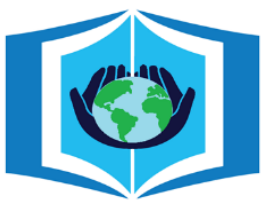

\section{URNCST Journal}

"Research in Earnest"

\section{Funded by the Government of Canada}

Canadà̀

Do you research in earnest? Submit your next undergraduate research article to the URNCST Journal!

| Open Access | Peer-Reviewed | Rapid Turnaround Time | International |

| Broad and Multidisciplinary | Indexed | Innovative | Social Media Promoted |

Pre-submission inquiries? Send us an email at info@ urncst.com | Facebook, Twitter and LinkedIn: @URNCST

Submit YOUR manuscript today at https://www.urncst.com 